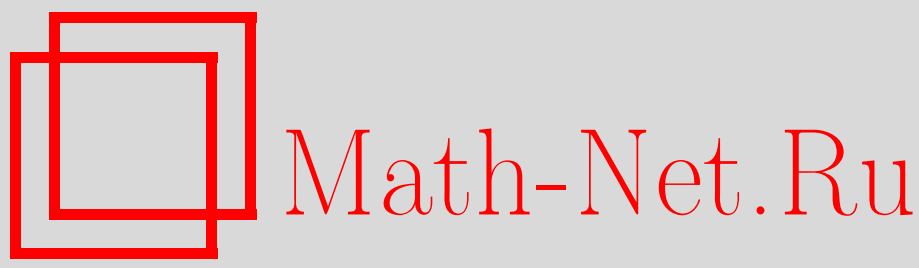

Л. В. Кузьмин, О формулах для числа классов вещественных абелевых полей, Изв. РАН. Сер. матем., 1996, том 60, выпуск 4, 43-110

DOI: https://doi.org/10.4213/im79

Использование Общероссийского математического портала Math-Net.Ru подразумевает, что вы прочитали и согласны с пользовательским соглашением http://www . mathnet.ru/rus/agreement

Параметры загрузки:

IP : 54.89 .56 .158

26 апреля 2023 г., 18:17:37 
УДК 519.4

\author{
Л.В. Кузьмин
}

\title{
О формулах для числа классов вещественных абелевых полей
}

\begin{abstract}
Для заданных вещественного абелева поля $k$ и простого натурального $\ell$ получена индексформула для порядка группы $\mathrm{Cl}(k)_{\ell, \varphi}$, где $\mathrm{Cl}(k)_{\ell}-\ell$-компонента группы классов поля $k$ и $\mathrm{Cl}(k)_{\ell, \varphi}$ означает $\varphi$-компоненту группы $\mathrm{Cl}(k)_{\ell}$, соответствующую $\mathbf{Q}_{\ell}$-неприводимому характеру $\varphi$ группы Галуа $G(k / \mathbf{Q})$, тривиальному на силовской $\ell$-подгруппе группы $G(k / \mathbf{Q})$. Этот результат обобщает гипотезу Гра. Доказательства основаны на использовании "основной гипотезы" теории Ивасавы.

Библиограффия: 17 наименований.
\end{abstract}

\section{Введение}

Пусть $k$ - вешественное абелево поле с группой классов $\mathrm{Cl}(k)$ и числом классов $h$. Если $k$ имеет вид $k=\mathbf{Q}\left(\varepsilon_{\ell}+\varepsilon_{\ell}^{-1}\right)$, т.е. $k$ является максимальным вешественным подполем кругового поля $\mathbf{Q}\left(\varepsilon_{\ell}\right)$, где $\varepsilon_{\ell}$ - первообразный корень из единицы степени $\ell$ и $\ell$ - простое число, то еще Куммер доказал, что $h$ равно индексу подгруппы круговых единиц $C(k)$ в группе всех единиц $U(k)$.

Этот результат был обобщен рядом авторов на некоторые другие типы вещественных абелевых полей и другие типы круговых единищ (см. ссылки в [16]). Наиболее важные результаты в этом направлении были получены Синноттом. В [15] он доказал, что в случае, когда $k$ является максимальным вешественным подполем некоторого кругового поля $\mathbf{Q}\left(\varepsilon_{m}\right)$, т.е. $k=\mathbf{Q}\left(\varepsilon_{m}+\varepsilon_{m}^{-1}\right)$, справедливо равенство

$$
[U(k): C(k)]=2^{b} h(k) \text {. }
$$

Здесь $C(k)$ - группа круговых единиц поля $k$ в смысле Синнотта (ниже в этом разделе мы дадим ее определение), а $b$ - неотрищательное целое число, зависящее только от числа $g$ различных простых делителей $m$. Именно,

$$
b=0 \text { при } g=1 \text { и } b=2^{g-2}-g+1 \text { при } g>1 .
$$

Синнотт получил также формулу

$$
[U(k): C(k)]=c_{k}^{+} h(k)
$$

которая справедлива для любого абелева вещественного поля [16]. В этом случае группа круговых единищ $C(k)$ и дополнительный множитель $c_{k}^{+}$определяются более сложным способом. Доказательство формул (0.1) и (0.3), данное Синноттом, основано на использовании аналитической формулы для числа классов. Поэтому его доказательство ничего не говорит нам о соотношении между двумя абелевыми группами $U(k) / C(k)$ и $\mathrm{Cl}(k)$ даже в случае, когда эти группы имеют одинаковые 
порядки. Однако хорошо известно, что в обшем случае эти группы не изоморфны. Например, если $k$ - вешественное квадратичное поле, то группа $U(k) / C(k)$ всегда циклическая, в то время как группа $\mathrm{Cl}(k)$ не является циклической в общем случае. Тем не менее Ж. Гра [2] предположил, что для любого вещественного абелева поля $k$ и любого простого $\ell$, взаимно простого со степенью $[k: \mathbf{Q}]$, $\ell$-компоненты групп $\mathrm{Cl}(k)$ и $U(k) / C(k)$ имеют изоморфные композиционные ряды Жордана-Гёльдера. Впервые эта гипотеза была полностью доказана в [17]. Следует отметить, что недавно В. А. Колывагин [6] получил относительно простое доказательство этой гипотезы в случае, когда $k=\mathbf{Q}\left(\varepsilon_{\ell}+\varepsilon_{\ell}^{-1}\right)$.

Настояшая работа возникла из желания достичь лучшего понимания взаимосвязи между группами $\mathrm{Cl}(k)$ и $U(k) / C(k)$ для произвольного вещественного абелева поля $k$. В частности, мы получили уточнение формул Синнотта $(0.1)$ и $(0.3)$. Чтобы сформулировать наши основные результаты, прежде всего заметим, что мы можем рассматривать только " $\ell$-компоненты" формул $(0.1)$ и $(0.3)$ для произвольного, но фиксированного простого $\ell$. Например, формула (0.1) эквивалентна утверждению, что для любого простого $\ell$ порядки $\ell$-компонент $(U(k) / C(k))_{\ell}$ и $\mathrm{Cl}(k)_{\ell}$ совпадают (с учетом дополнительного множителя $2^{b}$ для $\ell=2$ ). Мы пользуемся чисто $\ell$-адическими методами, поэтому в дальнейшем мы предполагаем, что все рассматриваемые поля являются подполями некоторого фиксированного алгебраического замыкания $\overline{\mathbf{Q}}_{\ell}$ поля рациональных $\ell$-адических чисел $\mathbf{Q}_{\ell}$, а все рассматриваемые характеры принимают значения в $\overline{\mathbf{Q}}_{\ell}$. Пусть $G=G(k / \mathbf{Q})$ и $G=G_{\ell} \times G_{0}$, где $G_{\ell}$ - силовская $\ell$-подгруппа группы $G$, а порядок группы $G_{0}$ взаимно прост с $\ell$. Пусть $\varphi$ - некоторый $\mathbf{Q}_{\ell}$-неприводимый характер группы $G_{0}$. Тогда для любого $\mathbf{Z}_{\ell}[G]$-модуля $A$ имеется разложение $A=\bigoplus_{\varphi \in \Phi} A_{\varphi}$, где $\varphi$ пробегает

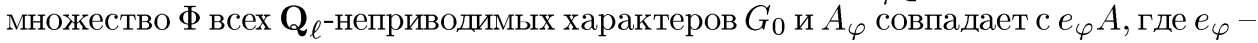
идемпотент, соответствующий $\varphi$. Через $\varphi_{0}$ мы обозначим тривиальный характер группы $G_{0}$. Для любой конечно порожденной абелевой группы $B$ через $B[\ell]$ мы обозначаем про- $\ell$-пополнение $B$. Мы доказываем следуюшие результаты.

ТЕОРЕМА 1. Пусть $k$ - максимальное вещественное подполе некоторого кругового поля $\mathbf{Q}\left(\varepsilon_{m}\right)$. Тогда для любого простого $\ell$ и любого характера $\varphi \in \Phi$ справедливо равенство

$$
\left[U(k)[\ell]_{\varphi}: C(k)[\ell]_{\varphi}\right]=\lambda\left|\operatorname{Cl}(k)_{\ell, \varphi}\right|
$$

əдe

$$
\lambda=\left\{\begin{array}{lllll}
1 & n p u & \ell \neq 2 \\
1 & n p u & \ell=2 & & \\
2^{b} & n p u & \ell=2 & u & \varphi=\varphi_{0} \\
& & &
\end{array}\right.
$$

$a b$ задается формулой (0.2).

ТЕОРема 2. Пусть $k$ - произвольное вещественное абелево поле. Тогда для любого простого $\ell$ и любого $\varphi \in \Phi$ справедливо равенство

$$
\left[U(k)[\ell]_{\varphi}: C(k)[\ell]_{\varphi}\right]=c_{k, \varphi}^{+}\left|\mathrm{Cl}(k)_{\ell, \varphi}\right|
$$

с некоторым рациональным $c_{k, \varphi}^{+}$, причем определение множителя $c_{k, \varphi}^{+}$, которое будет дано ниже, не зависит явно от арифметики поля $k$.

Позже мы покажем, что $c_{k, \varphi}^{+}$в некотором естественном смысле можно рассматривать как $\varphi$-компоненту константы Синнотта $c_{k}^{+}$. Поэтому $(0.4)$ и $(0.5)$ можно 
рассматривать как уточнение формул (0.1) и (0.3). Отметим, что гипотеза Гра эквивалентна утверждению, что для $G=G_{0}$ имеет место равенство

$$
\left[U(k)[\ell]_{\varphi}: C(k)[\ell]_{\varphi}\right]=\left|\mathrm{Cl}(k)_{\ell, \varphi}\right|
$$

Таким образом, мы можем рассматривать теоремы 1 и 2 как обобщение гипотезы Гра.

Оказывается, что теория Ивасавы является основным инструментом, позволяюшим доказать эти теоремы и некоторые другие связанные с ними результаты.

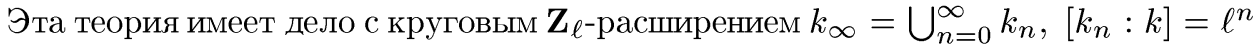
заданного поля $k=k_{0}$, где $\ell$ - некоторое фиксированное простое число. Для любого такого расширения определен модуль Тэйта (модуль Ивасавы) $T_{\ell}\left(k_{\infty}\right)$, которьй можно определить как $\lim \mathrm{Cl}\left(k_{n}\right)_{S, \ell}$. Здесь $\mathrm{Cl}\left(k_{n}\right)$ совпадает с группой классов поля $k_{n}, \mathrm{Cl}\left(k_{n}\right)_{S}$ обозначает факторгруппу группы $\mathrm{Cl}\left(k_{n}\right)$ по подгруппе, порожденной всеми простыми дивизорами $v \in S$, где $S$ - множество всех точек поля, лежащих над $\ell$, индекс $\ell$ означает переход к $\ell$-компоненте и проективньй предел берется относительно норменных отображений. Глобальная теория полей классов позволяет нам интерпретировать $T_{\ell}\left(k_{\infty}\right)$ как группу Галуа максимального абелева неразветвленного $\ell$-расширения $M_{\infty}$ поля $k_{\infty}$ такого, что все точки поля $k_{\infty}$, лежашие над $\ell$, вполне распадаются в $M_{\infty} / k_{\infty}$. Группа Галуа $T_{\ell}\left(k_{\infty}\right)=G\left(M_{\infty} / k_{\infty}\right)$ является абелевой про- $\ell$-группой и $\Gamma$-модулем относительно действия группы $\Gamma=G\left(k_{\infty} / k\right) \cong \mathbf{Z}_{\ell}$.

Рассмотрим группу $T_{\ell}\left(k_{\infty}\right)_{(0)}=T_{\ell}\left(k_{\infty}\right) /(\gamma-1)$, где $\gamma$ - некоторая фиксированная топологическая образуюшая групшы $\Gamma$. Группу $T_{\ell}\left(k_{\infty}\right)_{(0)}$ можно интерпретировать как группу Галуа $G\left(M_{\infty}^{0} / k_{\infty}\right)$, где $M_{\infty}^{0}$ - максимальное подполе поля $M_{\infty}$, абелево над $k$. Хорошо известно, что гипотеза Леопольдта справедлива для любого вешественного абелева поля, поэтому $T_{\ell}\left(k_{\infty}\right)_{(0)}$ является конечной абелевой $\ell$-группой.

Нашим первым основным результатом является некоторая индексформула, даюшая порядок групп $T_{\ell}\left(k_{\infty}\right)_{(0)}$ и $T_{\ell}\left(k_{\infty}\right)_{(0), \varphi}$ для любого $\varphi \in \Phi$.

ТЕОРема 3. Для любого вещественного абелева поля $k$ и любого $\varphi \in \Phi$ справедливы равенства

$$
\begin{aligned}
\left|T_{\ell}\left(k_{\infty}\right)_{(0)}\right| & =\left[\widehat{U}_{S}(k): C_{S}(k)\right], \\
\left|T_{\ell}\left(k_{\infty}\right)_{(0), \varphi}\right| & =\left[\widehat{U}_{S}(k)_{\varphi}: C_{S}(k)_{\varphi}\right] .
\end{aligned}
$$

Здесь $\widehat{U}_{S}(k)$ означает некоторую модифицированнул группу единиц, а $C_{S}(k)$ означает некоторую модифичированную группу круговых единич. Полное определение этих групп будет приведено ниже.

Заметим, что формулировка теоремы 3 не содержит никаких дополнительных множителей. Мы докажем теорему 3 с помощью теории Ивасавы, а затем выведем теоремы 1 и 2 из теоремы 3.

Теперь мы дадим полное определение групп $\widehat{U}_{S}(k)$ и $C_{S}(k)$. Затем мы более подробно опишем наш метод и содержание работы, отмечая наиболее важные промежуточные результаты.

Для произвольного поля $k$ пусть $k^{*}$ - мультипликативная группа поля $k, \mu(k)-$ группа всех корней из единицы в $k$ и $\mu_{\ell}(k)$ означает $\ell$-компоненту группы $\mu(k)$. 
Для поля алгебраических чисел $k$ пусть $k_{v}$ означает $v$-пополнение $k$ относительно некоторой точки $v \mid \ell$. Тогда мы положим

$$
\mathcal{B}\left(k_{v}\right):=\left(k_{v}^{*} / \mu\left(k_{v}\right)\right)[\ell] \quad \text { и } \quad \mathcal{B}(k):=\prod_{v \mid \ell} \mathcal{B}\left(k_{v}\right) .
$$

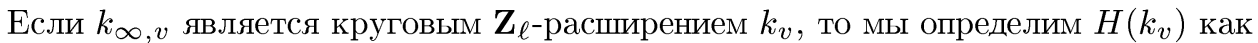
подгруппу всех универсальных норм в группе $\mathcal{B}\left(k_{v}\right)$ относительно расширения $k_{\infty, v} / k_{v}$, т.е.

$$
H\left(k_{v}\right)=\bigcap_{n=1}^{\infty} N_{k_{n, v} / k_{v}} \mathcal{B}\left(k_{n, v}\right)
$$

Положим

$$
H(k):=\prod_{v \mid \ell} H\left(k_{v}\right) .
$$

Тогда из локальной теории полей классов следует, что группа $H\left(k_{v}\right)$ (соответ-

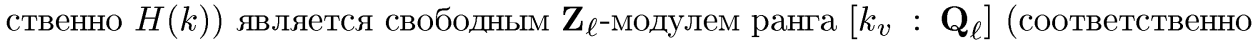
ранга $[k: \mathbf{Q}])$.

Для абелева поля $k$ обозначим через $U_{S}(k)$ группу $S$-единиц поля $k$, где $S$ было определено вьше. Положим $\bar{U}_{S}(k)=U_{S}(k) / \mu(k), \bar{U}(k)=U(k) / \mu(k)$. Поскольку гипотеза Леопольдта справедлива для поля $k$, естественное отображение $\bar{U}_{S}(k)[\ell] \rightarrow \mathcal{B}(k)$ инъективно, поэтому мы можем рассматривать $\bar{U}_{S}(k)[\ell]$ как подгруппу группы $\mathcal{B}(k)$. Положим

$$
\widehat{U}_{S}(k):=\bar{U}_{S}(k)[\ell] \cap H(k) .
$$

Мы можем считать, что $\widehat{U}_{S}(k)$ - это подгруппа групшы $\bar{U}_{S}(k)[\ell]$, состояшая из всех локальных универсальных норм из поля $k_{\infty}$.

Чтобы определить для абелева поля $k$ группу круговых $S$-единищ $C_{S}(k)$, мы заметим, что $k$ является подполем некоторого кругового поля $K=\mathbf{Q}\left(\varepsilon_{m}\right)$. Мы можем предположить, что $K$ содержит первообразньй корень из единицы степени $\ell$ $(K$ содержит $\sqrt{-1}$ при $\ell=2)$. Тогда любое промежуточное подполе $K_{n}$ кругового

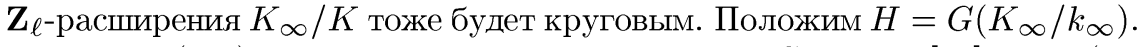

Пусть $P\left(K_{n}\right)$ - группа круговых чисел в смысле Синнотта [15], т.е. $P\left(K_{n}\right)-$ подгруппа групшы $K_{n}^{*}$, порожденная всеми числами вида $1-\varepsilon, \varepsilon \in \mu\left(K_{n}\right), \varepsilon \neq 1$. Тогда Синнотт определяет круговые единицы поля $K_{n}$ формулой $C\left(K_{n}\right):=P\left(K_{n}\right) \cap$ $U\left(K_{n}\right)$, где $U\left(K_{n}\right)$ - группа единиц поля $K_{n}$. Положим

$$
C\left(K_{\infty}\right):=\lim _{\longleftarrow}\left(C\left(K_{n}\right) / \mu\left(K_{n}\right)[\ell]\right), \quad \bar{U}_{S}\left(K_{\infty}\right):=\lim _{\longleftarrow}\left(\bar{U}_{S}\left(K_{n}\right)[\ell]\right)
$$

и

$$
\bar{U}_{S}\left(k_{\infty}\right):=\lim _{\longleftarrow}\left(\bar{U}_{S}\left(k_{n}\right)[\ell]\right),
$$

где все проективные пределы берутся относительно норменных отображений. Мы рассматриваем $C\left(K_{\infty}\right)$ как подгруппу группы $H\left(K_{\infty}\right):=\lim _{\longleftarrow} \mathcal{B}\left(K_{n}\right)=\lim _{\longleftarrow} H\left(K_{n}\right)$. Мы определим групшы $\bar{U}_{S}\left(k_{\infty}\right)$ и $H\left(k_{\infty}\right)$ аналогичным образом. Естественное вложение $k_{\infty} \subset K_{\infty}$ индуцирует вложения $H\left(k_{\infty}\right) \subset H\left(K_{\infty}\right), \bar{U}_{S}\left(k_{\infty}\right) \subset \bar{U}_{S}\left(K_{\infty}\right)$. Пусть

$$
\widetilde{C}\left(k_{\infty}\right):=C\left(K_{\infty}\right)^{H}, \quad \widetilde{H}\left(k_{\infty}\right):=H\left(K_{\infty}\right)^{H} .
$$


Положим

$$
\begin{aligned}
& \widetilde{C}_{S}\left(k_{\infty}\right):=\left\{x \in \widetilde{H}\left(k_{\infty}\right) \mid\left(\gamma_{n}-1\right) x \in \widetilde{C}\left(k_{\infty}\right) \text { при некотором натуральном } n\right\}, \\
& \text { где } \gamma_{n}=\gamma^{\ell^{n}}, \text { и } \\
& \qquad C_{S}\left(k_{\infty}\right)=\widetilde{C}_{S}\left(k_{\infty}\right) \cap \bar{U}_{S}\left(k_{\infty}\right) .
\end{aligned}
$$

Имеется естественная проекция

$$
\pi: \bar{U}_{S}\left(k_{\infty}\right) \longrightarrow \bar{U}_{S}(k)[\ell],
$$

и очевидно, что $\operatorname{Im} \pi \subset \widehat{U}_{S}(k)$. Мы определим $S$-круговые единицы поля $k$ формулой

$$
C_{S}(k):=\pi\left(C_{S}\left(k_{\infty}\right)\right) \subset \widehat{U}_{S}(k) .
$$

Отметим, что все определенные нами группы являются модулями Галуа. Более того, группы $H\left(K_{\infty}\right), \bar{U}_{S}\left(K_{\infty}\right), C\left(K_{\infty}\right)$ и т.д. имеют естественное строение $R_{\infty}$-модулей, где $R_{\infty}:=\lim \mathbf{Z}_{\ell}\left[G\left(K_{n} / \mathbf{Q}\right)\right]$ - пополненное групповое кольцо группы Галуа $G_{\infty}:=G\left(K_{\infty} / \overleftarrow{\mathbf{Q}}\right)$. Сушествует естественное разложение в прямое произведение $G\left(K_{\infty} / \mathbf{Q}\right)=\Gamma \times V$, где $\Gamma=G\left(\mathbf{Q}_{\infty} / \mathbf{Q}\right)$ и $V=G(E / \mathbf{Q}), E$ - максимальное подполе поля $K$, кондуктор которого не делится на $\ell^{2}$ (не делится на $\ell^{3}$ при $\ell=2)$. Мы имеем $R_{\infty}=\Lambda[V]$, где $\Lambda=\mathbf{Z}_{\ell}[[\Gamma]] \cong \lim \left[\Gamma / \Gamma_{n}\right]$ и $\Gamma_{n}-$ единственная подгруппа группы $\Gamma$ индекса $\ell^{n}$. Зафиксировав топологическую образующую $\gamma \in \Gamma$, мы получаем изоморфизм $\Lambda \cong \mathbf{Z}_{\ell}[[T]], \gamma \rightarrow 1+T$, где $\mathbf{Z}_{\ell}[[T]]-$ кольцо формальных степенных рядов от одной переменной $T$ над $\mathbf{Z}_{\ell}$.

В $\S 1$ мы даем характеризацию группы $C\left(K_{\infty}\right)$ как $\Lambda$-модуля. Используя результаты Кубера [7], [8] об ординарных универсальных распределениях, мы доказываем, что $C\left(K_{\infty}\right)$ является свободным $\Lambda$-модулем (теорема 1.1 ).

В $\S 2$ мы рассматриваем характеристические ряды конечно порожденных $\Lambda$-периодических $R_{\infty}$-модулей $A$. В случае, когда $A$ свободен над $\mathbf{Z}_{\ell}$, мы получаем формулу, выражающую порядок $\left|A_{(0), \varphi}^{H}\right|=\left|A_{\varphi,(0)}^{H}\right|$ в терминах характеристических рядов $A$ (предложение 2.3 ), где $H$ - некоторая подгруппа группы $V$. Затем мы даем описание характеристических рядов $R_{\infty}$-модуля $\mathcal{A}\left(K_{\infty}\right) / C\left(K_{\infty}\right)$, где $\mathcal{A}\left(K_{\infty}\right):=\lim _{\longleftarrow} \mathcal{A}\left(K_{n}\right)$ и $\mathcal{A}\left(K_{n}\right):=\prod_{v \mid \ell} \bar{U}\left(K_{n, v}\right), \bar{U}\left(K_{n, v}\right)=U\left(K_{n, v}\right) / \mu\left(K_{n, v}\right)$. Это описание основано на теореме Ивасавы о круговых единищах [4,] [11], [12]. Мы показываем, что рассматриваемые характеристические ряды тесно связаны с так называемыми рядами Ивасавы.

В 33 мыпродолжаем вычисление характеристических рядов. Пусть $T_{\ell}\left(K_{\infty}\right)^{+}$ максимальная подгруппа групшы $T_{\ell}\left(K_{\infty}\right)$, неподвижная относительно действия автоморфизма комплексного сопряжения. Положим $\bar{U}\left(K_{\infty}\right)=\lim _{\longleftarrow} \bar{U}\left(K_{n}\right)[\ell]$. Мы доказываем (теорема 3.1$)$, что модули $T_{\ell}\left(K_{\infty}\right)^{+}$и $\bar{U}\left(K_{\infty}\right) / C\left(K_{\infty}\right)$ имеют одинаковые характеристические ряды для любого четного характера группы $V$. Доказательство основывается на так назьваемой "основной гипотезе теории Ивасавы” [4], [13], [17], а также на некоторых теоремах двойственности, полученных автором в [10]. Затем мы обобщаем этот результат на случай произвольного абелева числового поля $k$ и его кругового $\mathbf{Z}_{\ell}$-расширения $k_{\infty}$.

В $\S 4$ мы доказываем теорему 3 введения. Коротко говоря, если $k$-вешественное абелево поле, то существует точная последовательность

$$
0 \longrightarrow P \longrightarrow T_{\ell}\left(k_{\infty}\right) \longrightarrow R \longrightarrow 0
$$


где $P$ - максимальный конечный подмодуль модуля $T_{\ell}\left(k_{\infty}\right)$ и модуль $R$ свободен над $\mathbf{Z}_{\ell}$. Мы получаем из $(0.9)$, что

$$
\left|T_{\ell}\left(k_{\infty}\right)_{(0), \varphi}\right|=\left|P_{(0), \varphi}\right| \cdot\left|R_{(0), \varphi}\right|
$$

для любого $\varphi$. С другой стороны, обозначая через $U_{S}^{*}(k)$ образ $\pi\left(\bar{U}_{S}\left(k_{\infty}\right)\right)$ в формуле (0.8), мы получаем вложения $C_{S} \subseteq U_{S}^{*}(k) \subseteq \widehat{U}_{S}(k)$. Таким образом, мы имеем

$$
\left|\widehat{U}_{S}(k)_{\varphi} / C_{S}(k)_{\varphi}\right|=\left[\widehat{U}_{S}(k)_{\varphi}: U_{S}^{*}(k)_{\varphi}\right] \cdot\left[U_{S}^{*}(k)_{\varphi}: C_{S}(k)_{\varphi}\right]
$$

для любого $\varphi \in \Phi$. Используя характеристические ряды, мы доказываем, что

$$
\left|U_{S}^{*}(k)_{\varphi}: C_{S}(k)_{\varphi}\right|=\left|R_{(0), \varphi}\right|
$$

для любого $\varphi$.

В $[9$, предложение 7.5$]$ было доказано сушествование естественного изоморфизма

$$
\widehat{U}_{S}(k) / U_{S}^{*}(k) \cong T_{\ell}\left(k_{\infty}\right)^{\Gamma} .
$$

Мы даем в $\S 4$ короткое независимое доказательство (0.13) (см. предложение 4.3). Тогда теорема 3 непосредственно следует из (0.10)-(0.13).

Чтобы вывести теоремы 1 и 2 из теоремы 3 , нам необходима некоторая модификация теоремы 3 . Пусть $\nu_{\ell}$ означает $\ell$-адический показатель в $\overline{\mathbf{Q}}_{\ell}$, нормализованный так, чтобы выполнялось условие $\nu_{\ell}(\ell)=1$. В $\S 5$ нами доказывается

ТЕОРема 4. Для любого вещественного абелева поля $k$ и любого характера $\varphi \in \Phi$ справедливо равенство

$$
\nu_{\ell}\left(\left|T_{\ell}\left(k_{\infty}\right)_{(0), \varphi}\right|\right)=\nu_{\ell}\left(\left[H(k)_{\varphi}: \widehat{U}_{S}(k)_{\varphi}\right]^{-1} \ell^{t_{\varphi}} \prod_{\substack{\chi \neq 1 \\ \chi \mid \varphi}} \frac{1}{2} L_{\ell}(1, \chi)\right),
$$

где $\chi$ пробегает все нетривиальные одномерные характеры группы $G=$ $G(k / \mathbf{Q})$ такие, что ограничение $\left.\chi\right|_{G_{0}}$ входит в $\varphi, t_{\varphi}-$ некоторый множитель, определенный явно в теореме 5.1, a $L_{\ell}(s, \chi)$ означает $\ell$-адическую $L$-функиию Kуботь-Леопольдта [5].

Отметим, что справедливо равенство

$$
\left[\widehat{U}_{S}(k)_{\varphi}: C_{S}(k)_{\varphi}\right]=\left[H(k)_{\varphi}: \widehat{U}_{S}(k)_{\varphi}\right]^{-1}\left[H(k)_{\varphi}: C_{S}(k)_{\varphi}\right]
$$

поэтому единственное отличие данной ситуации от ситуации теоремы 3 состоит в том, что мы даем явную формулу для индекса $\left[H(k)_{\varphi}: C_{S}(k)_{\varphi}\right]$. Эта формула получена нами из рассмотрения характеристических рядов модуля $H\left(k_{\infty}\right) / C_{S}\left(k_{\infty}\right)$. Эти ряды тесно связаны с рядами Ивасавы. Отметим, что открытая Ивасавой [5] связь между рядами Ивасавы и $\ell$-адическими $L$-функциями объясняет, почему (0.14) содержит значения функции $L_{\ell}(1, \chi)$.

В $\S 6$ мы доказываем один результат, который можно рассматривать как уточнение теоремы Хассе о дискриминанте и ведушем модуле. Для абелева поля алгебраических чисел $k$ рассмотрим алгебру Галуа $\mathbf{A}_{k}=k \otimes_{\mathbf{Q}} \overline{\mathbf{Q}}_{\ell}$. Пусть $\mathbf{R}_{k}$-максимальный порядок алгебры $\mathbf{A}_{k}$. Естественное вложение $k \hookrightarrow \mathbf{A}_{k}$ индуцирует вложение $\alpha: \mathcal{O}_{k} \hookrightarrow \mathbf{R}_{k}$, где $\mathcal{O}_{k}-$ кольцо цельх поля $k$. Положим $\mathcal{O}_{k}=\overline{\mathcal{O}} \cdot \alpha\left(\mathcal{O}_{k}\right) \subset \mathbf{R}_{k}$, где $\overline{\mathcal{O}}$ - кольцо целых поля $\overline{\mathbf{Q}}_{\ell}$. Тогда выполняется (теорема 6.1) 
ТЕОРема 5. Для любого $\varphi \in \Phi$ справедливо равенство

$$
\nu_{\ell}\left(\left(\mathbf{R}_{k, \varphi}: \widetilde{\mathcal{O}}_{k, \varphi}\right)\right)=\nu_{\ell}\left(\prod_{\substack{\chi \neq 1 \\ \chi \mid \varphi}} g_{\bar{\chi}}\right)
$$

где $(A: B)$ означает обобщенный индекс в смысле Синнотта [15], а $g_{\bar{\chi}}-$ гауссова сумма, соответствующая характеру $\bar{\chi}=\chi^{-1}$.

В $\S 7$ мы вычисляем некоторые индексы единиц. Пусть $k$ - вешественное абелево поле и $G=G(k / \mathbf{Q})$. Как и в $[15]$, для $\mathbf{Z}_{\ell}[G]$-модуля $A$, свободного над $\mathbf{Z}_{\ell}$, мы полагаем

$$
A_{0}=\left\{x \in A \mid \operatorname{Sp}_{G}(x)=0\right\},
$$

где $\mathrm{Sp}_{G}$ - отображение следа относительно группы $G$. Мы доказываем (теорема 7.1), что для любого $\varphi \in \Phi$

$$
\ell^{s_{\varphi}} \frac{\left|T_{\ell}\left(k_{\infty}\right)_{(0), \varphi}\right|}{\left|\mathrm{Cl}(k)_{\ell, \varphi}\right|}=\frac{\left[\mathcal{A}(k)_{0, \varphi}: \bar{U}(k)[\ell]_{\varphi}\right]}{\left[H(k)_{\varphi}: \widehat{U}_{S}(k)_{\varphi}\right]}
$$

где $s_{\varphi}=0$ при $\varphi \neq \varphi_{0}$ и $s_{\varphi_{0}}-$ некоторая простая константа, явно указанная в предложении 7.4.

Пусть $\log : \bar{U}(k)[\ell] \hookrightarrow \mathbf{A}_{k}-$ композиция отображений $\log : \bar{U}(k)[\ell] \hookrightarrow k \otimes_{\mathbf{Q}} \mathbf{Q}_{\ell}$, индуцированного отображением $\ell$-адического логарифма, и естественного вложения $k \otimes_{\mathbf{Q}} \mathbf{Q}_{\ell} \hookrightarrow \mathbf{A}_{k}=k \otimes_{\mathbf{Q}} \overline{\mathbf{Q}}_{\ell}$. Положим $\widetilde{U}(k)=\overline{\mathcal{O}} \log (\bar{U}(k)[\ell])$. Следуюшая теорема (теорема 7.3) может рассматриваться как $\ell$-адическая аналитическая формула для числа классов некоторой $\varphi$-компоненты $\ell$-группы классов.

ТЕОРема 6. Для произвольного вещественного абелева поля $k$ и любого характера $\varphi \in \Phi$ справедливо равенство

$$
\nu_{\ell}\left(\left|\mathrm{Cl}(k)_{\ell, \varphi}\right|\right)=\nu_{\ell}\left(\left(\mathbf{R}_{k, 0, \varphi}: \widetilde{U}(k)_{\varphi}\right)^{-1} \prod_{\substack{\chi \in \widehat{G} \\ \chi \mid \varphi, \chi \neq 1}} \frac{1}{2} a(\chi)\right)
$$

əдe

$$
a(\chi)=\sum_{\substack{a \bmod f_{\chi} \\\left(a, f_{\chi}\right)=1}} \bar{\chi}(a) \log \left(1-\varepsilon_{\chi}^{a}\right)
$$

$f_{\chi}$ - кондуктор $\chi, \varepsilon_{\chi}-$ первообразный корень из единицы степени $f_{\chi} u \log$ означает $\ell$-адический логарифм.

Отметим, что индекс $\left(\mathbf{R}_{k, 0, \varphi}: \widetilde{U}(k)_{\varphi}\right)$ можно интерпретировать как $\varphi$-компоненту $\ell$-адического регулятора поля $k$.

Мы выводим теорему 6 из теорем 4 и 5 и из (0.15). Затем, используя метод Синнотта, мы получаем (теорема 7.4) "абстрактную индексформулу". Эта формула выражает порядок $\left|\mathrm{Cl}(k)_{\ell, \varphi}\right|$ в терминах индекса $\left[\bar{U}(k)[\ell]_{\varphi}: C_{k, \varphi}\right]$, где группа круговых единищ $C_{k}$ определяется аксиоматически.

В $\S 8$ мы, используя аргументы Синнотта, выводим теорему 1 из теоремы 7.4 (см. теорему 8.3). 
В $\S 9$ мы доказываем теорему 2 (см. теорему 9.2). Затем мы доказываем формулу для числа классов еще одного типа (см. теорему 9.3). Эта формула содержит модифицированную группу круговых единищ и модифицированные константы $c_{k, \varphi}^{+}$. В некоторых частных случаях удается явно вычислить эти модифицированные константы, что приводит к нетривиальным сравнениям для числа классов некоторых вешественных абелевых полей (см. теоремы 9.4 и 9.5). Отметим, что похожие (и даже более сильные) результаты были получены Корнеллом [1] с помощью теории родов.

В $\S 10$ мы даем некоторые замечания к нашим результатам и формулируем ряд открытых проблем.

Автор глубоко благодарен профессору Х. Коху и его коллегам по рабочей группе "Алгебраическая геометрия и теория чисел" при институте Макса Планка, чье гостеприимство помогло автору выполнить значительную часть данной работы.

Автор благодарит также М. Ж. Шматикова, научившего его пользоваться системой LAT $\mathrm{E}$, и Н. В. Игумнову за помощь в подготовке рукописи.

\section{§1. О круговых единицах в $\mathrm{Z}_{\ell}$-расширениях}

Пусть $\ell$ - фиксированное простое число и $d \not \equiv 2(\bmod 4)$ - некоторое натуральное число, взаимно простое с $\ell$. Мы считаем $d$ фиксированным на протяжении всего параграфа. Пусть $\mu_{d}$ - группа всех корней из единицы степени $d$ в некотором фиксированном алгебраическом замыкании $\overline{\mathbf{Q}}$ поля $\mathbf{Q}$. Мы полагаем $K_{-1}:=\mathbf{Q}\left(\mu_{d}\right)$ и $K_{n}:=\mathbf{Q}\left(\mu_{d}, \zeta_{n}\right)$ при любом $n \geqslant 0$, где $\zeta_{n} \in \overline{\mathbf{Q}}-$ первообразный корень из единицы степени $\ell^{n+1}$ (степени $\ell^{n+2}$ при $\left.\ell=2\right)$. Мы выберем корни $\zeta_{n}$ таким образом, чтобы при любом $n$ выполнялось равенство $\zeta_{n+1}^{\ell}=\zeta_{n}$. Тогда любое круговое поле имеет вид $K_{n}$ для некоторых $d$ и $n$, причем при фиксированном $d$ поля $K_{n}$, $n \geqslant 0$, образуют башню всех промежуточных подполей кругового $\mathbf{Z}_{\ell}$-расширения $K_{\infty} / K_{0}$, где по определению $K_{\infty}:=\bigcup_{n=1}^{\infty} K_{n}$. Мы обозначаем через $\mu\left(K_{n}\right)$ (соответственно через $\left.\mu_{\ell}\left(K_{n}\right)\right)$ группу всех корней из единицы в поле $K_{n}$ (соответственно $\ell$-компоненту групшы $\left.\mu\left(K_{n}\right)\right)$.

Пусть $P\left(K_{n}\right)$ и $C\left(K_{n}\right)$ - определенные во введении групшы круговых чисел и круговых единищ соответственно. Заметим, что элементы $\left(1-\alpha \zeta_{n}^{i}\right)$, где $\alpha \in \mu_{d}$, $i=0, \ldots, \ell^{n+1+\delta}-1$, порождают всю группу $P\left(K_{n}\right)$. Если это не приводит к недоразумению, мы будем обозначать эти группы просто через $P_{n}$ и $C_{n}$, опуская символ $K$. Мы полагаем $P_{\infty}:=\lim \bar{P}_{n}[\ell], C_{\infty}:=\lim \bar{C}_{n}[\ell]$, где $\bar{P}_{n}:=$ $P_{n} / \mu\left(K_{n}\right), \bar{C}_{n}:=C_{n} /\left(\mu\left(K_{n}\right) \cap C_{n}\right)$ и символ [ $\left.\ell\right]$ означает $\ell$-пополнение. Таким образом, как было отмечено во введении, групшы $P_{\infty}$ и $C_{\infty}$ являются $R_{\infty}$-модулями и $\Lambda$-модулями.

Для $\alpha \in \mu_{d}$ мы положим $\varepsilon_{n}(\alpha):=1-\alpha^{\ell^{-n-\delta}} \zeta_{n}$, где $\delta=0$ при $\ell \neq 2$ и $\delta=1$ при $\ell=2$. Элементы $\varepsilon_{n}(\alpha)$ образуют при $n \geqslant 0$ последовательность, согласованную относительно норменных отображений. Следовательно, мы имеем элемент

$$
\varepsilon_{\infty}(\alpha):=\lim _{\longleftarrow} \varepsilon_{n}(\alpha) \in P_{\infty}
$$

ПРЕДЛОЖЕНИЕ 1.1. Элементы $\varepsilon_{\infty}(\alpha)$, где $\alpha$ пробегает $\mu_{d}$, порождают $P_{\infty}$ как $R_{\infty}$-модуль. 
ДокАЗАТЕльСтво. Пусть $D_{n}-$ подмодуль Галуа в группе $\bar{P}_{n}$, порожденный всеми элементами $\varepsilon_{n}(\alpha)$ для $\alpha \in \mu_{d}$. Так как $N_{K_{n} / K_{r}}\left(\varepsilon_{n}(\alpha)\right)=\varepsilon_{r}(\alpha)$ для $n>$ $r \geqslant 0$, мы имеем $\bar{P}_{n}=D_{n} \cdot \bar{P}_{-1}$. Следовательно,

$$
P_{\infty}=\lim _{\longleftarrow} \bar{P}_{n}[\ell]=\lim _{\longleftarrow} D_{n}[\ell]
$$

Чтобы исследовать строение групп $P_{\infty}$ и $C_{\infty}$, как модулей Галуа, нам потребуются некоторые результаты об универсальных распределениях, полученные в [7], [8]. По определению универсальным распределением на группе $\frac{1}{N} \mathbf{Z} / \mathbf{Z}$ для некоторого натурального $N$ называется абелева группа $U(N)$, порожденная символами $(a)$ для всех $a \in \frac{1}{N} \mathbf{Z} / \mathbf{Z}$, удовлетворяюшими соотношениям

$$
\sum_{m b=a}(b)=(a) \text { для любого } a \in \frac{1}{N} \mathbf{Z} / \mathbf{Z}, \quad m \mid N .
$$

Согласно [7] группа $U(N)$ является свободной абелевой группой. Естественное действие групшы $G(N)=(\mathbf{Z} / N \mathbf{Z})^{*}$ на $\frac{1}{N} \mathbf{Z} / \mathbf{Z}$ индуцирует действие $G(N)$ на $U(N)$, и мы имеем изоморфизм $G(N)$-модулей $[7$, теорема 4.11$]$

$$
U(N) \otimes \mathbf{z} \mathbf{Q} \cong \mathbf{Q}[G(N)] .
$$

Если $N \mid N_{1}$, то естественное включение $\frac{1}{N} \mathbf{Z} / \mathbf{Z} \hookrightarrow \frac{1}{N_{1}} \mathbf{Z} / \mathbf{Z}$ и отображение $\frac{1}{N_{1}} \mathbf{Z} / \mathbf{Z} \rightarrow \frac{1}{N} \mathbf{Z} / \mathbf{Z}$, переводящее $x \in \frac{1}{N_{1}} \mathbf{Z} / \mathbf{Z}$ в $\frac{N_{1}}{N} x \in \frac{1}{N} \mathbf{Z} / \mathbf{Z}$, индуцируют отображения $i\left(N, N_{1}\right): U(N) \rightarrow U\left(N_{1}\right)$ и $N\left(N_{1}, N\right): U\left(N_{1}\right) \rightarrow U(N)$. Хорошо известно, что отображение $i\left(N, N_{1}\right)$ всегда инъективно. Из (1.1) немедленно следует

ПРЕДЛОЖЕНИЕ 1.2. При произвольном простом $\ell$ пусть $N \equiv 0(\bmod \ell)$. Пусть $N_{1}=\ell N$. Обозначим через $H\left(N_{1}, N\right)$ ядро естественной проекиии $G\left(N_{1}\right) \rightarrow G(N)$. Тогда отображсение $i\left(N, N_{1}\right) \circ N\left(N_{1}, N\right): U\left(N_{1}\right) \rightarrow U\left(N_{1}\right)$ совпадает с норменным отображением относительно группь $H\left(N_{1}, N\right)$.

Для произвольного натурального $N$ пусть $Z^{*}(N)$ - множество всех примитивных элементов группы $\frac{1}{N} \mathbf{Z} / \mathbf{Z}$, т.е. элементов, имеющих порядок в точности $N$. Пусть $N=\prod_{p \mid N} p^{n(p)}$ - разложение $N$ на простые множители. Тогда имеется естественное разложение

$$
\frac{1}{N} \mathbf{Z} / \mathbf{Z}=\prod_{p \mid N}\left(\frac{1}{p^{n(p)}} \mathbf{Z} / \mathbf{Z}\right), \quad Z^{*}(N)=\prod_{p \mid N} Z^{*}\left(p^{n(p)}\right) .
$$

Tеорема A [7, теорема 1.8, предложение 1.9]. Пусть

$$
T(N):=\prod_{p \mid N}\left[Z^{*}\left(p^{n(p)}\right)-\left\{\frac{1}{p^{n(p)}}\right\} \cup\{0\}\right] .
$$

Тогда әлементы $(t)$ для $t \in T(N)$ образуют базис свободной абелевой группье $U(N)$.

Пусть $\ell$ - простое число, $d$ взаимно просто с $\ell$ и $d \not \equiv 2(\bmod 4)$. Положим

$$
U\left(\ell^{\infty} d\right):=\lim _{\longleftarrow}\left(U\left(\ell^{n} d\right) \otimes \mathbf{z} \mathbf{Z}_{\ell}\right),
$$

где проективный предел берется относительно отображений $N\left(\ell^{n+1} d, \ell^{n} d\right)$. Мы имеем естественное действие группы $G_{\infty}:=\lim G\left(\ell^{n} d\right)$ на $U\left(\ell^{\infty} d\right)$. Пусть $\Gamma$ - ядро естественной проекции $G_{\infty} \rightarrow G(q d)$, где $q=\ell$ при $\ell \neq 2$ и $q=4$ при $\ell=2$. Тогда группа $\Gamma \cong \mathbf{Z}_{\ell}$ действует на $U\left(\ell^{\infty} d\right)$. Таким образом, $U\left(\ell^{\infty} d\right)$ имеет естественное строение $\Lambda$-модуля. 
ПРЕДЛОЖЕНИЕ 1.3. Группа $U\left(\ell^{\infty} d\right)$ является свободным $\Lambda$-модулем ранга $\varphi(q d)$, где $\varphi(x)-$ функиия Эйлера.

ДокАЗАТЕЛЬСТво. Для $N=\ell^{n} d, d=\prod_{p \mid d} p^{n(p)}$, мы представим множество $T\left(\ell^{n} d\right)$ в виде несвязного объединения

$$
T\left(\ell^{n} d\right)=T\left(\ell^{n} d\right)_{1} \cup T\left(\ell^{n} d\right)_{2},
$$

где

$$
\begin{aligned}
& T\left(\ell^{n} d\right)_{1}:=\left[Z^{*}\left(\ell^{n}\right)-\left\{\frac{1}{\ell^{n}}\right\}\right] \times \prod_{p \mid d}\left[Z^{*}\left(p^{n(p)}\right)-\left\{\frac{1}{p^{n(p)}}\right\} \cup\{0\}\right], \\
& T\left(\ell^{n} d\right)_{2}:=\{0\} \times \prod_{p \mid d}\left[Z^{*}\left(p^{n(p)}\right)-\left\{\frac{1}{p^{n(p)}}\right\} \cup\{0\}\right] .
\end{aligned}
$$

Поскольку существует разложение $\frac{1}{\ell^{n} d} \mathbf{Z} / \mathbf{Z} \cong \frac{1}{\ell^{n}} \mathbf{Z} / \mathbf{Z} \oplus \frac{1}{d} \mathbf{Z} / \mathbf{Z}$, любой элемент $a \in$ $\frac{1}{\ell^{n} d} \mathbf{Z} / \mathbf{Z}$ может быть однозначно представлен в виде $a=\left(a^{\prime}, a^{\prime \prime}\right)$, где $a^{\prime} \in \frac{1}{\ell^{n}} \mathbf{Z} / \mathbf{Z}$, $a^{\prime \prime} \in \frac{1}{d} \mathbf{Z} / \mathbf{Z}$. Если $H_{n} \cong\left(\mathbf{Z} / \ell^{n} \mathbf{Z}\right)^{*}$ - ядро естественной проекции $G\left(\ell^{n} d\right) \rightarrow G(d)$, то любые два элемента $a, b \in \frac{1}{\ell^{n} d} \mathbf{Z} / \mathbf{Z}$ вида $a=\left(a^{\prime}, a^{\prime \prime}\right), \quad b=\left(b^{\prime}, b^{\prime \prime}\right)$ сопряжены относительно действия $H_{n}$ тогда и только тогда, когда $a^{\prime \prime}=b^{\prime \prime}$ и $a^{\prime}, b^{\prime}$ имеют один и тот же порядок как элементы группы $\frac{1}{\ell^{n}} \mathbf{Z} / \mathbf{Z}$. Это означает, что $\mathbf{Z}_{\ell}\left[G\left(\ell^{n} d\right)\right]$-подмодуль $U_{1}\left(\ell^{n} d\right)$ модуля $U\left(\ell^{n} d\right) \otimes \mathbf{z} \mathbf{Z}_{\ell}$, порожденньй всеми $(t), t \in$ $T\left(\ell^{n} d\right)_{1}$, может быть порожден как $H_{n}$-модуль множеством $\varphi(d)$ элементов $(t)$, где $t$ пробегает множество

$$
T\left(\ell^{n} d\right)_{3}:=\left\{\frac{1}{\ell^{n}}\right\} \times \prod_{p \mid d}\left[Z^{*}\left(p^{n(p)}\right)-\left\{\frac{1}{p^{n(p)}}\right\} \cup\{0\}\right] .
$$

Если мы перейдем к проективному пределу относительно отображений $N\left(\ell^{n+1} d, \ell^{n} d\right)$, то получим, что модуль $U_{1}\left(\ell^{\infty} d\right):=\lim U_{1}\left(\ell^{n} d\right)$ может быть порожден $\varphi(d)$ элементами как $H_{\infty}$-модуль, где $H_{\infty}:=\longleftarrow \lim _{n} \cong \mathbf{Z}_{\ell}^{*}$. Следовательно, $U_{1}\left(\ell^{\infty} d\right)$ может быть порожден $\varphi(q) \varphi(d)=\varphi(q d)$ элементами как $\Lambda$-модуль. Пусть $U_{2}\left(\ell^{n} d\right)-\mathbf{Z}_{\ell}\left[G\left(\ell^{n} d\right)\right]$-подмодуль модуля $U\left(\ell^{n} d\right) \otimes \mathbf{z} \mathbf{Z}_{\ell}$, порожденньй всеми $(t)$, где $t$ пробегает множество $T\left(\ell^{n} d\right)_{2}$. Тогда, рассуждая, как и при доказательстве предложения 1.1 , мы получаем, что

$$
U\left(\ell^{\infty} d\right):=\lim _{\longleftarrow}\left(U_{1}\left(\ell^{n} d\right) \cdot U_{2}\left(\ell^{n} d\right)\right)=\lim _{\longleftarrow} U_{1}\left(\ell^{n} d\right)=U_{1}\left(\ell^{\infty} d\right) .
$$

Таким образом, существует сюръективное отображение $\Lambda$-модулей $\alpha: \Lambda^{\varphi(q d)} \rightarrow$ $U\left(\ell^{\infty} d\right)$. Чтобы проверить, что $U\left(\ell^{\infty} d\right)$ является свободным $\Lambda$-модулем ранга $\varphi(q d)$, заметим, что естественная проекция $p_{n}: U_{1}\left(\ell^{\infty} d\right) \rightarrow U_{1}\left(\ell^{n} d\right)$ сюръективна для любого $n$. Сюръективное отображение $p_{n} \circ \alpha: \Lambda^{\varphi(q d)} \rightarrow U_{1}\left(\ell^{n} d\right)$ индуцирует сюръекцию $\beta_{n}: \Lambda_{(n-\delta-1)}^{\varphi(q d)} \rightarrow U_{1}\left(\ell^{n} d\right)$, где $\delta=0$ при $\ell \neq 2$ и $\delta=1$ при $\ell=2$, причем для любого $G_{\infty}$-модуля $A$ через $A_{(i)}$ обозначается результат факторизации $A$ по действию подгруппы $\Gamma_{i} \subseteq \Gamma$ индекса $\ell^{i}$ (таким образом, $\Gamma_{n-\delta-1}$ совпадает с ядром естественного отображения $\left.G_{\infty} \rightarrow G\left(\ell^{n} d\right)\right)$. Обозна-

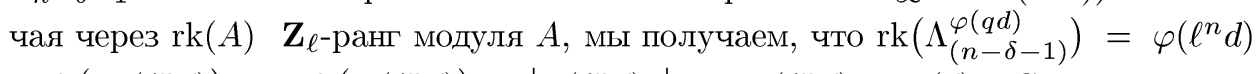
и $\operatorname{rk}\left(U_{1}\left(\ell^{n} d\right)\right) \geqslant \operatorname{rk}\left(U\left(\ell^{n} d\right)\right)-\left|T\left(\ell^{n} d\right)_{2}\right|=\varphi\left(\ell^{n} d\right)-\varphi(d)$. Следовательно, $\operatorname{rk}\left(\operatorname{Ker} \beta_{n}\right) \leqslant \varphi(d)$, поэтому $\operatorname{Ker} \alpha=\lim \operatorname{Ker} \beta_{n}=0$. Предложение доказано. 
Теорема В [8, теорема 2.16, предложение 3.3]. Пусть $\{ \pm \mathrm{id}\} \subset G(N)-$ nодгруппа, порожденная әлементами \pm 1 . Тогда для любого $k$ существует изоморфизм $H^{k}( \pm \mathrm{id}, U(N)) \cong(\mathbf{Z} / 2 \mathbf{Z})^{r}$, где $H^{k}($,$) - группь когомологий в$ смысле Тэйта, $r=2^{\nu(N)-1} u \nu(N)$ - число различных простых делителей $N$. Пусть $M \mid N, \nu(M)=\nu(N)$ и $M \not \equiv 2(\bmod 4) ; \operatorname{mozда~включение~}$ $i(M, N): U(M) \hookrightarrow U(N)$ индуцирует изоморфизм

$$
i^{*}(M, N): H^{k}( \pm \mathrm{id}, U(M)) \cong H^{k}( \pm \mathrm{id}, U(N))
$$

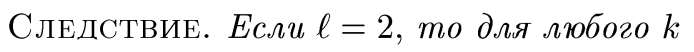

$$
H^{k}\left( \pm \mathrm{id}, U\left(\ell^{\infty} d\right)\right)=0 .
$$

Действительно, по теореме В при $\ell=2$ норменное отображение относительно $H\left(\ell^{n} d, \ell^{n-1} d\right)$ совпадает с умножением на $\left|H\left(\ell^{n} d, \ell^{n-1} d\right)\right|=2$ для $n \geqslant 3$ и, следовательно, обращает в нуль группы $H^{k}\left( \pm \mathrm{id}, U\left(\ell^{n} d\right)\right)$ при любом $k$. Тогда из предложения 1.2 и из изоморфного характера отображений $i^{*}\left(\ell^{n-1} d, \ell^{n} d\right)$ следует, что отображение $N\left(\ell^{n} d, \ell^{n-1} d\right)$ обращает в нуль группы $H^{k}\left( \pm \mathrm{id}, U\left(\ell^{n} d\right)\right)$ при любых $k$ и $n \geqslant 3$. Следовательно,

$$
H^{k}\left( \pm \mathrm{id}, U\left(\ell^{\infty} d\right)\right)=0
$$

TеОрема 1.1. Группь $P_{\infty}$ и $C_{\infty}$ являются свободными $\Lambda$-модулями ранга $\frac{1}{2}\left[K_{0}: \mathbf{Q}\right]$.

ДокАЗАТЕЛЬСТво. Пусть $\alpha$ - некоторая фиксированная образующая группы $\mu_{d}$. Для произвольного элемента

$$
\frac{c}{\ell^{n+1+\delta} d} \in \frac{1}{\ell^{n+1+\delta} d} \mathbf{Z} / \mathbf{Z}, \quad c \in \mathbf{Z}
$$

положим

$$
\psi^{n}(0)=1, \quad \psi^{n}\left(\frac{c}{\ell^{n} d}\right)=\left(1-\alpha^{\ell^{-n} c} \zeta_{n}^{c}\right) \quad\left(\bmod P_{n} \cap\left(\mathbf{Q}^{*} \times \mu\left(K^{n}\right)\right)\right) .
$$

Тогда мы получаем отображение

$$
\psi^{n}: \frac{1}{\ell^{n+1+\delta} d} \mathbf{Z} / \mathbf{Z} \rightarrow P_{n} / P_{n} \cap\left(\mathbf{Q}^{*} \times \mu\left(K_{n}\right)\right) .
$$

Отображение $\psi^{n}$ является четным распределением в смысле [7], т.е. оно удовлетворяет соотношениям

$$
\sum_{m b=a} \psi^{n}(b)=\psi^{n}(a) \text { для любого } a \in \frac{1}{\ell^{n+1+\delta} d} \mathbf{Z} / \mathbf{Z}, \quad m \mid \ell^{n+1+\delta} d
$$

и

$$
\psi^{n}(-a)=\psi^{n}(a)
$$

Следовательно, отображение $\psi^{n}$ определяет естественный эпиморфизм

$$
\bar{\psi}^{n}: U\left(\ell^{n+1+\delta} d\right) \otimes_{\mathbf{z}} \mathbf{Z}_{\ell} \rightarrow P_{n} / P_{n} \cap\left(\mathbf{Q}^{*} \times \mu\left(K_{n}\right)\right)[\ell] .
$$


Отображения $\bar{\psi}^{n}$ согласованы с действием групп $G\left(\ell^{n+1+\delta} d\right) \cong G\left(K_{n} / \mathbf{Q}\right)$ и с норменньми отображениями, поэтому, переходя к проективному пределу относительно норменных отображений, мы получаем эпиморфизм $R_{\infty}$-модулей

$$
\bar{\psi}_{\infty}: U\left(\ell^{\infty} d\right) / U\left(\ell^{\infty} d\right)^{-} \longrightarrow \lim _{(}\left(P_{n} / P_{n} \cap\left(\mathbf{Q}^{*} \times \mu\left(K_{n}\right)\right)[\ell]\right)=P_{\infty},
$$

где $U\left(\ell^{\infty} d\right)^{-}$означает максимальную подгруппу группы $U\left(\ell^{\infty} d\right)$, на которую -id действует как умножение на -1 .

Поскольку $P_{\infty}$ можно рассматривать как $\Lambda$-подмодуль $R_{\infty}$-модуля $H\left(K_{\infty}\right)$, определенного во введении, мы получаем, что $P_{\infty}$ не имеет $\Lambda$-кручения. Из предложения 1.3 и определения группы $U\left(\ell^{\infty} d\right)^{-}$следует, что модуль $U\left(\ell^{\infty} d\right) / U\left(\ell^{\infty} d\right)^{-}$ также не имеет $\Lambda$-кручения. Пусть $U\left(\ell^{\infty} d\right)^{+}$означает максимальную подгруппу группы $U\left(\ell^{\infty}\right)$, неподвижную относительно действия группы $\{ \pm \mathrm{id}\}$. Тогда легко проверить, что оба модуля, $U\left(\ell^{\infty} d\right)^{+}{ }_{\text {и }} U\left(\ell^{\infty} d\right)^{-}$, имеют $\Lambda$-ранг $\frac{1}{2}\left[K_{0}: \mathbf{Q}\right]$.

Согласно предложению 1.1 для любого $n$ сушествует естественный эпиморфизм $P_{\infty} \rightarrow D_{n}[\ell]$. Согласно $[15$, теорема 4.1$]$ группа $C_{n}$ имеет конечный индекс в группе единиц $U\left(K_{n}\right)$, поэтому мы получаем, что $\mathbf{Z}_{\ell}$-ранг групшы $D_{n}[\ell]$ удовлетворяет при любом $n$ условию

$$
\operatorname{rk} D_{n}[\ell] \geqslant \operatorname{rk} U\left(K_{n}\right)[\ell]-\operatorname{rk} \bar{P}_{-1}[\ell],
$$

т.е. $\operatorname{rk} D_{n}[\ell] \geqslant \frac{1}{2}\left[K_{n}: \mathbf{Q}\right]-c$, где $c$ не зависит от $n$. Тогда из [9, предложение 1.2$]$ следует, что $\Lambda$-ранг модуля $P_{\infty}$ равен $\frac{1}{2}\left[K_{0}: \mathbf{Q}\right]$, т.е. отображение $\bar{\psi}_{\infty}$ является изоморфизмом.

Если $\ell \neq 2$, то $U\left(\ell^{\infty} d\right)=U\left(\ell^{\infty} d\right)^{+} \oplus U\left(\ell^{\infty} d\right)^{-}$, поэтому $\bar{\psi}_{\infty}$ индуцирует изоморфизм $U\left(\ell^{\infty} d\right)^{+} \cong P_{\infty}$. Следовательно, в этом случае $P_{\infty}$ свободен как $\Lambda$-модуль.

При $\ell=2$ мы имеем изоморфизм

$$
P_{\infty} \cong U\left(\ell^{\infty} d\right) / U\left(\ell^{\infty} d\right)^{-} \cong N\left(U\left(\ell^{\infty} d\right)\right)
$$

где $N$ ( ) означает образ норменного отображения относительно группы $\{ \pm \mathrm{id}\}$. Coгласно следствию из теоремы В $H^{0}\left(\{ \pm \mathrm{id}\}, U\left(\ell^{\infty} d\right)\right)=0$, следовательно, $N\left(U\left(\ell^{\infty} d\right)\right)=U\left(\ell^{\infty} d\right)^{+}$. Чтобы доказать, что $U\left(\ell^{\infty} d\right)^{+}$свободен как $\Lambda$-модуль, заметим, что $U\left(\ell^{\infty} d\right)^{+}$является подмодулем $\Lambda$-свободного модуля $U\left(\ell^{\infty} d\right)$, кроме того, $U\left(\ell^{\infty} d\right) / U\left(\ell^{\infty} d\right)^{+}$не имеет кручения, поэтому наше утверждение вытекает из $[9$, предложение 1.1].

Итак, мы показали, что в любом случае $P_{\infty}$ является $\Lambda$-свободным модулем. Чтобы показать, что $C_{\infty} \Lambda$-свободен, заметим, что произвольный $x \in \bar{P}_{n}[\ell]$ может содержаться в образе естественной проекции $P_{\infty} \rightarrow \bar{P}_{n}[\ell]$ только в том случае, когда $x$ принадлежит $\ell$-пополнению группь $S$-единищ, где $S$ состоит из всех простых делителей $\ell$. Пусть $\varepsilon_{m}-$ первообразньй корень из единицы степени $m$. Хорошо известно, что число $1-\varepsilon_{m}$ является единицей тогда и только тогда, когда число $m$ составное. Если $m=p^{r}$ для некоторого простого $p$, то $1-\varepsilon_{p^{r}}$ является простым элементом локального поля $\mathbf{Q}_{p}\left(\varepsilon_{p^{r}}\right)$. Тогда легко проверить, что точна последовательность $\Lambda$-модулей

$$
0 \longrightarrow C_{\infty} \longrightarrow P_{\infty} \longrightarrow \mathbf{Z}_{\ell} \longrightarrow 0
$$

где $\mathbf{Z}_{\ell}$ порожден элементом $\varepsilon_{\infty}(1)$. Поскольку $P_{\infty}$ свободен над $\Lambda$, из $[9$, предложение 1.1] следует, что $C_{\infty}$ также свободен над $\Lambda$, что доказывает теорему. 


\section{§2. Некоторые характеристические ряды}

Пусть $R_{\infty}:=\mathbf{Z}_{\ell}\left[\left[G_{\infty}\right]\right]:=\lim \mathbf{Z}_{\ell}\left[G\left(K_{n} / \mathbf{Q}\right)\right]$ - кольцо, определенное во введении. Имеется естественное разложение в прямое произведение $G_{\infty}:=$ $G\left(K_{\infty} / \mathbf{Q}\right)=\Gamma \times V$, где $\Gamma \cong G\left(\mathbf{Q}_{\infty} / \mathbf{Q}\right) \cong G\left(K_{\infty} / K_{0}\right), V \cong G\left(K_{0} / \mathbf{Q}\right) \cong$ $G\left(K_{\infty} / \mathbf{Q}_{\infty}\right)$. Если мы зафиксируем некоторую топологическую образуюшую $\gamma \in \Gamma$, то получим изоморфизм $\Lambda:=\mathbf{Z}_{\ell}[[\Gamma]] \cong \mathbf{Z}_{\ell}[[T]], \gamma \rightarrow 1+T$, где $\mathbf{Z}_{\ell}[[T]]$ - кольцо формальных степенных рядов. Тогда $R_{\infty}=\Lambda[V]$. Мы предполагаем, что все $R_{\infty}$-модули, рассматриваемые ниже, конечно порождены над $R_{\infty}$. Если $\chi$ - некоторый одномерный характер $V$, принимающий значения в алгебраическом замыкании $\overline{\mathbf{Q}}_{\ell}$ поля $\mathbf{Q}_{\ell}$, то кольцо $\mathcal{O}_{\chi}$, порожденное над $\mathbf{Z}_{\ell}$ всеми значениями характера $\chi$, получит естественную структуру $V$-модуля, если мы положим $\sigma(a)=\chi(\sigma) a$ для любых $\sigma \in V, a \in \mathcal{O}_{\chi}$. Для любого $R_{\infty}$-модуля $A$ мы определим $\chi$-компоненту $A$ формулой

$$
A_{\chi}=A \otimes_{\mathbf{z}_{\ell}[V]} \mathcal{O}_{\chi}
$$

Таким образом, $R_{\infty, \chi} \cong \mathcal{O}_{\chi}[[\Gamma]] \cong \mathcal{O}_{\chi}[[T]]$. Мы можем рассматривать $A_{\chi}$

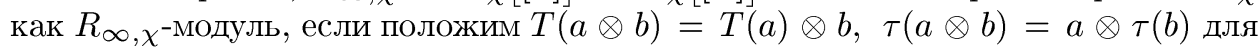
$a \in A, b, \tau \in \mathcal{O}_{\chi}$. Положим $V=V_{\ell} \times V_{0}$, где $V_{\ell}$ - силовская $\ell$-подгруппа групшы $V$ и $\left(\left|V_{0}\right|, \ell\right)=1$. Пусть $\Phi$-множество всех $\mathbf{Q}_{\ell}$-неприводимых характеров группы $V_{0}$. Тогда для любого $R_{\infty}$-модуля $A$ имеется разложение в прямое произведение

$$
A=\bigoplus_{\varphi \in \Phi} A_{\varphi}
$$

где $A_{\varphi}=e_{\varphi} A$ и $e_{\varphi}$ - идемпотент, соответствуюший $\varphi$. Для одномерного характера $\chi \in \widehat{V}$, где $\widehat{V}$ - группа всех характеров группы $V$, запись $\chi \mid \varphi$ означает, что ограничение $\left.\chi\right|_{V_{0}}$ характера $\chi$ на $V_{0}$ входит в $\varphi$ как его неприводимая компонента над $\overline{\mathbf{Q}}_{\ell}$. Мы имеем

$$
\left(A_{\varphi}\right)_{\chi}= \begin{cases}A_{\chi} \cong A_{\varphi} & \text { при } \chi \mid \varphi \\ 0 & \text { в противном случае. }\end{cases}
$$

Если $A$ является $\Lambda$-периодическим модулем, то определен характеристический ряд $f_{A}=f_{A}(T) \in \Lambda$ модуля $A$ (см. [5], [12]). Мы напомним некоторые свойства таких рядов. Прежде всего, заметим, что $f_{A}$ определен однозначно с точностью до умножения на некоторую единицу $u \in \Lambda^{*}$. Если $A, B$ - квазиизоморфные $\Lambda$-периодические модули (т.е. существует гомоморфизм $f: A \rightarrow B$ с конечным ядром и коядром; мы пишем в этом случае $A \sim B$ ), то $f_{A}=f_{B}$. Если $A=\bigoplus_{i=1}^{r} \Lambda / f_{i} \Lambda$ для некоторых $f_{i} \in \Lambda, f_{i} \neq 0$, то $f_{A}=\prod_{i=1}^{r} f_{i}$. Известно, что любой $\Lambda$-периодический модуль $A$ квазиизоморфен модулю вида $\bigoplus_{i=1}^{r} \Lambda / f_{i} \Lambda$.

Пусть $\Lambda$-модуль $A$ конечно порожден над $\mathbf{Z}_{\ell}$ (в данной работе мы будем иметь дело только с таким случаем). Тогда $f_{A}$ имеет вид $f_{1}(T) u$, где $u \in \Lambda^{*}$ и $f_{1}(T)-$ отмеченный многочлен вида $T^{\lambda}+a_{\lambda-1} T^{\lambda-1}+\cdots+a_{0}$, где $a_{\lambda-1}, \ldots, a_{0} \in \ell \mathbf{Z}_{\ell}$. Заметим, что мы можем рассматривать $f_{1}(T)$ как характеристический многочлен оператора $T=\gamma-1$, действуюшего на $\lambda$-мерном $\mathbf{Q}_{\ell}$-пространстве $A \otimes \mathbf{z}_{\ell} \mathbf{Q}_{\ell}$. Если $R_{\infty}$-модуль $A$ является $\Lambda$-периодическим, то для любого $\chi \in \widehat{V}$ существует характеристический ряд $f_{A}(T, \chi) \mathcal{O}_{\chi}[[T]]$-модуля $A_{\chi}$, определяемый аналогичным образом. Следуюшее утверждение хорошо известно. 
ПРЕДЛОЖЕНИЕ 2.1. Пусть $A-R_{\infty}$-модуль, который конечно порожден над $\mathbf{Z}_{\ell}$ и, следовательно, является $\Lambda$-периодическим. Тогда

$$
f_{A}(T)=\prod_{\chi \in \widehat{V}} f_{A}(T, \chi), \quad f_{A_{\varphi}}(T)=\prod_{\chi \mid \varphi} f_{A}(T, \chi)
$$

для любого $\varphi \in \Phi$, где мы полагаем $f_{A}(T, \chi)=1$ в случае, когда $A_{\chi}$ конечен. Пусть

$$
0 \longrightarrow A \longrightarrow B \longrightarrow C \longrightarrow 0
$$

- точная последовательность $R_{\infty}$-модулей, конечно порожденных над $\mathbf{Z}_{\ell}$. Тогда

$$
f_{B}=f_{A} \cdot f_{C}, \quad f_{B}(T, \chi)=f_{A}(T, \chi) \cdot f_{C}(T, \chi) \quad \text { для любого } \quad \chi \in \widehat{V} .
$$

Через $\nu_{\ell}\left(\right.$ ) мы будем обозначать $\ell$-адический показатель поля $\overline{\mathbf{Q}}_{\ell}$, нормированный условием $\nu_{\ell}(\ell)=1$.

ПреДЛОЖЕНИЕ 2.2. Пусть А является $\Lambda$-периодическим модулем. В этом случае группа $A_{(0)}=A /(\gamma-1) A$ конечна тогда и только тогда, когда $f_{A}(0) \neq 0$. Если модуль $А$ свободен над $\mathbf{Z}_{\ell}$, то

$$
\nu_{\ell}\left(\left|A_{(0)}\right|\right)=\nu_{\ell}\left(f_{A}(0)\right) \text {. }
$$

Доказательство. Условие $f_{A}(0) \neq 0$ означает, что группа $A^{\Gamma}$ конечна. Группа $A^{\Gamma}$ конечна тогда и только тогда, когда конечна группа $A_{(0)}$. Если $A, B-$

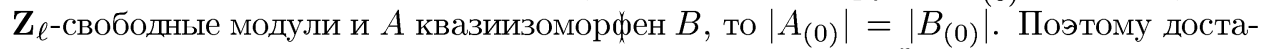
точно проверить формулу (2.4) для модуля $A$ вида $A=\bigoplus_{i=1}^{r} \Lambda / f_{i} \Lambda$, где $f_{i}$-некоторые отмеченные многочлены. В последнем случае справедливость формулы (2.4) очевидна. Предложение доказано.

Следующее утверждение представляет из себя далеко идущее обобщение предложения 2.2. Пусть задан эпиморфизм $\tau: G_{\infty} \rightarrow G$ группы $G_{\infty}$ на некоторую конечную группу $G$. Тогда $\operatorname{Ker} \tau=H \times \bar{\Gamma}$, где $H=V \cap \operatorname{Ker} \tau$, причем группа $\bar{\Gamma} \cong \mathbf{Z}_{\ell}$ определена неканонически. Мы сталкиваемся с такой ситуацией в случае, когда $G=G(k / \mathbf{Q})$ для некоторого поля алгебраических чисел $k \subset K_{\infty}$. В этом случае мы имеем $H=G\left(K_{\infty} / k_{\infty}\right), \bar{\Gamma} \cong G\left(k_{\infty} / k\right)$. Заметим, что мы можем выбрать топологическую образуюшую $\bar{\gamma}$ группы $\bar{\Gamma}$ вида

$$
\bar{\gamma}=\gamma^{\ell^{r}} \sigma
$$

для некоторого $\sigma \in V$, причем целое $r \geqslant 0$ является инвариантом поля $k$. Если $R_{\infty}$-модуль $A$ является $\Lambda$-периодическим и $\mathbf{Z}_{\ell}$-свободным, то этими же свойствами обладает и $((V / H) \times \bar{\Gamma})$-модуль $A^{H}$.

ПРЕДЛОЖЕНИЕ 2.3. Если группа $A_{(0)}$ конечна, то такова же и группа $\left(A^{H}\right)_{(0)}:=A^{H} /(\bar{\gamma}-1)$, и ее порядок $\ell^{t}$ задается формулой

$$
t=\nu_{\ell}\left(\prod_{\chi \in \widehat{V / H}} \prod_{\zeta^{\ell^{r}}=1} f_{A}\left(\zeta \zeta_{\chi}^{-1}-1, \chi\right)\right),
$$


где $\zeta_{\chi}$ определяется условием

$$
\zeta_{\chi}^{\ell^{r}}=\chi(\sigma)
$$

$\zeta$ пробегает все корни из единиць степени $\ell^{r}$ и $\sigma, r$ задаются равенством (2.5). Пусть $\varphi$ - произвольный $\mathbf{Q}_{\ell}$-неприводимый характер группь $V / V \cap H$, и пусть $\ell^{t_{\varphi}}-$ порядок группь $\left(A^{H}\right)_{(0), \varphi}=\left(\left(A^{H}\right)_{\varphi}\right)_{(0)}$. Тогда

$$
t_{\varphi}=\nu_{\ell}\left(\prod_{\substack{\chi \in \overline{V / H} \\ \chi \mid \varphi}} \prod_{\zeta^{\ell^{r}}=1} f_{A}\left(\zeta \zeta_{\chi}^{-1}-1, \chi\right)\right)
$$

где $\zeta_{\chi}$ и $\zeta$ определены, как и выше.

ДокАЗАТЕЛЬСтво. Прежде всего заметим, что значение $\nu_{\ell}\left(f_{A}(0)\right)$ в $(2.4)$ равно $\nu_{\ell}\left(\prod_{i=1}^{\lambda} \alpha_{i}\right)$, где $\alpha_{1}, \ldots, \alpha_{\lambda}$ - все корни отмеченного мгогочлена $f_{1}(T)$, или, что эквивалентно, все собственные значения оператора $\gamma-1$ на $A \otimes \mathbf{z}_{\ell} \mathbf{Q}_{\ell}$. Теперь пусть $\alpha(\chi)_{1}, \ldots, \alpha(\chi)_{\lambda(\chi)}$ - корни отмеченного многочлена, соответствуюшего характеристическому ряду $f_{A}(T, \chi)$ для некоторого $\chi \in \widehat{G / H}$. Элемент $\sigma$, определенньй формулой $(2.5)$, действует на группу $\left(A^{H}\right)_{\chi}$ умножением на $\chi(\sigma)$, следовательно, оператор $\bar{\gamma}$, действуюший на $\mathbf{Q}_{\ell}$-пространство $\left(A^{H}\right)_{\chi} \otimes \mathbf{z}_{\ell} \mathbf{Q}_{\ell}$, имеет собственные значения $\left(1+\alpha(\chi)_{1}\right)^{\ell^{r}} \chi(\sigma), \ldots,\left(1+\alpha(\chi)_{\lambda(\chi)}\right)^{\ell^{r}} \chi(\sigma)$. Тогда согласно $(2.2)$ и предложению 2.2 мы получаем

$$
t=\nu_{\ell}\left(\prod_{\chi \in \overline{V / H}} \prod_{i=1}^{\lambda(\chi)}\left(\left(1+\alpha(\chi)_{i}\right)^{\ell^{r}} \chi(\sigma)-1\right)\right) .
$$

Поскольку выполняются равенства

$$
\begin{aligned}
\left(1+\alpha(\chi)_{i}\right)^{\ell^{r}} \chi(\sigma)-1 & =\prod_{\zeta^{\ell^{r}}=1}\left(\left(1+\alpha(\chi)_{i}\right) \zeta_{\chi}-\zeta\right)=\prod_{\zeta^{\ell^{r}}=1} \zeta_{\chi}\left(1+\alpha(\chi)_{i}-\zeta_{\chi}^{-1} \zeta\right) \\
f_{1}(T, \chi) & =\prod_{i=1}^{\lambda(\chi)}\left(T-\alpha(\chi)_{i}\right)
\end{aligned}
$$

мы получаем

$$
\begin{aligned}
t & =\nu_{\ell}\left(\eta \prod_{\chi \in \widehat{V / H}} \prod_{\zeta^{\ell}=1} \prod_{i=1}^{\lambda(\chi)}\left(\zeta_{\chi}^{-1} \zeta-1-\alpha(\chi)_{i}\right)\right) \\
& =\nu_{\ell}\left(\eta \prod_{\chi \in \widehat{V / H}} \prod_{\zeta^{\ell^{r}}=1} f_{A}\left(\zeta \zeta_{\chi}^{-1}-1, \chi\right)\right)
\end{aligned}
$$

где $\eta= \pm 1$. Это доказывает формулу (2.6). Доказательство формулы (2.7) полностью аналогично. Предложение доказано. 
СлЕДСТВИЕ. В ситуачии предложения 2.3 пусть $\left|A_{(n)}\right|=\ell^{t(n)},\left|A_{(n), \varphi}\right|=$ $\ell^{t(n)_{\varphi}}$, где $A_{(n)}:=A /\left(\gamma_{n}-1\right), \gamma_{n}=\gamma^{\ell^{n}}$. Тогда

$$
\begin{aligned}
t(n) & =\nu_{\ell}\left(\prod_{\chi \in \widehat{V}} \prod_{\zeta^{\ell^{n}}=1} f_{A}(\zeta-1, \chi)\right), \\
t(n)_{\varphi} & =\nu_{\ell}\left(\prod_{\substack{\chi \in \widehat{V} \\
\chi \mid \varphi}} \prod_{\zeta^{\ell^{n}}=1} f_{A}(\zeta-1, \chi)\right) .
\end{aligned}
$$

Чтобы доказать это, достаточно применить предложение 2.3 к эпиморфизму $\tau: G_{\infty}=V \times \Gamma \rightarrow V \times \Gamma / \Gamma_{n}$, где $\Gamma_{n}:=\left\langle\gamma_{n}\right\rangle$.

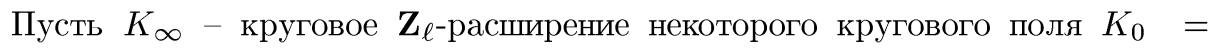
$\mathbf{Q}\left(\mu_{d}, \zeta_{0}\right)$. Напомним, что во введении были определены $R_{\infty}$-модули $\mathcal{A}\left(K_{\infty}\right)$ и $H\left(K_{\infty}\right)$ такие, что имеются естественные включения $C\left(K_{\infty}\right) \subset \mathcal{A}\left(K_{\infty}\right) \subset H\left(K_{\infty}\right)$. В дальнейшем мы будем работать с некоторым фиксированным полем $K_{\infty}$, поэтому вместо $C\left(K_{\infty}\right), \mathcal{A}\left(K_{\infty}\right)$ и т.д. мы будем писать просто $C_{\infty}, \mathcal{A}_{\infty}$ и т.д. Пусть $j \in G_{\infty}-$ автоморфизм комплексного сопряжения в поле $K_{\infty}$. Для произвольного $R_{\infty}$-модуля $A$ положим

$$
A^{+}=\{a \in A \mid j(a)=a\}, \quad A^{-}=\{a \in A \mid j(a)=-a\} .
$$

ПРЕДЛОЖЕНИЕ 2.4. Группь $\mathcal{A}_{\infty}, H_{\infty}$ являются свободными $\Lambda$-модулями ранга $\left[K_{0}: \mathbf{Q}\right]$. Группы $\mathcal{A}_{\infty}^{+}, H_{\infty}^{+}$являются свободными $\Lambda$-модулями ранza $\frac{1}{2}\left[K_{0}: \mathbf{Q}\right]$.

ДоКАЗАТЕЛЬСТво. Согласно [9, лемма 7.2 и предложение 7.1] $H_{\infty}$ является свободным $\Lambda$-модулем ранга $\left[K_{0}: \mathbf{Q}\right]$. Сушествует естественная точная последовательность

$$
0 \longrightarrow \mathcal{A}_{\infty} \longrightarrow H_{\infty} \longrightarrow \mathcal{D}_{\infty} \longrightarrow 0
$$

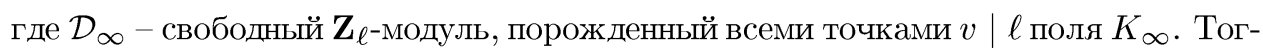
да, рассуждая, как в доказательстве теоремы 1.1 , мы получаем, что $\mathcal{A}_{\infty}$ также $\Lambda$-свободен. Поскольку $H_{\infty} / H_{\infty}^{+}$и $\mathcal{A}_{\infty} / \mathcal{A}_{\infty}^{+}$не имеют $\Lambda$-кручения, из $[9$, предложение 1.1] следует, что $H_{\infty}^{+}$и $\mathcal{A}_{\infty}^{+}$являются свободными $\Lambda$-модулями. Используя аргументы $[9, \S 7]$, можно легко доказать, что $H_{\infty}^{+}$и $\mathcal{A}_{\infty}^{+}$имеют $\Lambda$-ранг $\frac{1}{2}\left[K_{0}: \mathbf{Q}\right]$. Предложение доказано.

Теперь мы собираемся вычислить характеристические ряды $\Lambda$-периодического $R_{\infty}$-модуля $\mathcal{A}_{\infty} / C_{\infty}$. Для этого нам потребуются некоторые дополнительные обозначения и определения. Мы можем рассматривать любой характер $\chi \in \widehat{V}$, где $V=G\left(K_{0} / \mathbf{Q}\right)$, как примитивный характер Дирихле кондуктора $f_{\chi} \mid d q$, где $q=\ell$ при $\ell \neq 2$ и $q=4$ при $\ell=2$. Любой такой $\chi$ является характером первого рода в смысле Ивасавы [5]. Для любого такого $\chi$ определен идемпотент

$$
e_{\chi}=|V|^{-1} \sum_{\chi \in V} \bar{\chi}(\sigma) \sigma, \text { где } \bar{\chi}(\sigma)=\chi(\sigma)^{-1} .
$$

Пусть

$$
e^{+}=\frac{1+j}{2}, \quad e^{-}=\frac{1-j}{2} .
$$


Как и в [15, формула (3.3)], мы определяем "минус компоненту" элемента Штикельбергера $s\left(a, \ell^{n+1+\delta} d\right), a \neq \equiv 0\left(\bmod \ell^{n+1+\delta} d\right)$, соответствующего полю $K_{n}$ кондуктора $\ell^{n+1+\delta} d$, формулой

$$
e^{-} s\left(a, \ell^{n+1+\delta} d\right)=\sum_{\substack{i \bmod \ell^{n+1+\delta} \\(i, \ell d)=1}}\left(-\left\langle\frac{a i}{\ell^{n+1+\delta} d}\right\rangle+\frac{1}{2}\right) \sigma_{i}^{-1} \in \mathbf{Q}_{\ell}\left[G\left(K_{n} / \mathbf{Q}\right)\right]
$$

где $\langle x\rangle$ означает однозначно определенное рациональное число $x^{\prime}, 0 \leqslant x^{\prime}<1$, такое, что $x \equiv x^{\prime}(\bmod \mathbf{Z})$, а $\sigma_{i}$ - элемент группы $G\left(K_{n} / \mathbf{Q}\right)$, переводящий $\varepsilon$ в $\varepsilon^{i}$ для любого $\varepsilon \in \mu\left(K_{n}\right)$. Элементы $e^{-} s\left(a, \ell^{n+1+\delta} d\right)$ согласованы при различных $n$ относительно естественных проекций $\mathbf{Q}_{\ell}\left[G\left(K_{n_{2}} / \mathbf{Q}\right)\right] \rightarrow \mathbf{Q}_{\ell}\left[G\left(K_{n_{1}} / \mathbf{Q}\right)\right]$, где $n_{2}>n_{1}$. Таким образом, переходя к проективному пределу, мы получаем элемент

$$
s\left(a, \ell^{\infty} d\right):=\lim _{\longleftarrow} e^{-} s\left(a, \ell^{n+1+\delta} d\right) \in \lim _{\longleftarrow} \mathbf{Q}_{\ell}\left[G\left(K_{n} / \mathbf{Q}\right)\right] .
$$

Хорошо известно (см. [15, раздел 3]), что $\left(\sigma_{i}-i\right) \cdot s\left(a, \ell^{\infty} d\right) \in R_{\infty}$ для любого $i$, взаимно простого с $d \ell$. Поэтому мы можем рассматривать $s\left(a, \ell^{\infty} d\right)$ как элемент групповой алгебры $L[V]$, где $L$ - поле частных кольца $\Lambda$. Из данного в [5] определения рядов Ивасавы непосредственно следует, что в поле частных кольца $R_{\infty, \chi}=\mathcal{O}_{\chi}[[T]]$ справедливо равенство

$$
\frac{1}{2} e_{\chi} s\left(1, \ell^{\infty} f_{\chi}\right)=f(T, \chi)
$$

Здесь $\chi$-некоторый нечетный характер (т.е. $\chi(-1)=-1$ ) первого рода кондуктора $f_{\chi}$ и $f(T, \chi)$ - ряд Ивасавы, соответствуюший $\chi$. Отметим, что в [5] ряд $f(T, \chi)$ называется рядом, соответствуюшим четному характеру $\theta=\bar{\chi} \omega$, где $\omega$-характер Тейхмюллера, $\omega: V \rightarrow \mathbf{Z}_{\ell}^{*}, \omega\left(\sigma_{i}\right) \equiv i(\bmod q)$ для любого $\sigma_{i} \in V . \mathrm{B}[5]$ было доказано, что $f(T, \chi) \in \mathcal{O}_{\chi}[[T]]$ при $\chi \neq \omega$. Если $\chi=\omega$, то

$$
f(T, \omega)=\left(1-\frac{\kappa}{1+T}\right)^{-1} \eta(T, \omega)
$$

где $\eta(T, \omega)$ - обратимьй элемент кольца $\mathbf{Z}_{\ell}[[T]]$, и $\kappa \in 1+\mathbf{Z}_{\ell}$ определяется формулой $\gamma\left(\zeta_{n}\right)=\zeta_{n}^{\kappa}$ для любого $n \geqslant 0$. Положим

$$
Y\left(K_{n}\right):=\prod_{v \mid \ell} \mu_{\ell}\left(K_{n, v}\right)
$$

где $K_{n, v}$ - пополнение поля $K_{n}$ относительно $v$, и положим $Y_{\infty}:=\lim Y\left(K_{n}\right)$, где проективный предел берется относительно норменных отображений. Отметим, что, как $\mathbf{Z}_{\ell \text {-модуль, }} Y_{\infty}$ изоморфен модулю $\mathcal{D}_{\infty}$ из доказательства предложения 2.4. 
ТЕОРема 2.1. Пусть $h(T, \theta)$ (соответственно $y(T, \theta)$ ) - характеристический ряд $\Lambda$-периодического $R_{\infty}$-модуля $\mathcal{A}_{\infty}^{+} / C_{\infty}$ (соответственно модуля $\left.Y_{\infty}\right)$, соответствующий некоторому четному характеру первого рода $\theta$. Тогда, полагая $\bar{\theta}(\sigma)=\theta(\sigma)^{-1}$, мы имеем

$$
\begin{aligned}
h(T, \theta) & =f\left(\frac{\kappa}{1+T}-1, \bar{\theta} \omega\right) y(T, \theta)^{-1} \quad \text { npu } \quad \theta \neq 1 \\
h(T, \theta) & =\eta\left(\frac{\kappa}{1+T}-1, \omega\right) y(T, 1)^{-1} \\
& =(-T) f\left(\frac{\kappa}{1+T}-1, \omega\right) y(T, 1)^{-1} \quad \text { npu } \quad \theta=1 .
\end{aligned}
$$

ДокАЗАТЕЛьСтво. Мы вьведем формулы (2.11) и (2.12) из [11, теорема 4.1]. Чтобы сформулировать это последнее утверждение, введем некоторые определения. Обозначим через $S_{\infty}^{\prime} R_{\infty}$-модуль, порожденный в $L[V]$ элементами $s\left(a, \ell^{\infty} d\right)$ для всех $a \in \mathbf{Z} \backslash\{0\}$. Легко можно проверить, что $S_{\infty}^{\prime}$ порождается как $R_{\infty}$-модуль множеством $s\left(a, \ell^{\infty} d\right)$ для $a=1, \ldots, d q$. Следовательно, $S_{\infty}^{\prime}-$ конечно порожденный $R_{\infty}$-модуль. Мы определим идеал Штикельбергера в $R_{\infty}$ формулой $S_{\infty}:=$ $S_{\infty}^{\prime} \cap R_{\infty}$. В [11] для поля $K_{\infty}$ был определен $R_{\infty}$-модуль с одной образующей $V\left(K_{\infty}\right)$ такой, что $\mathcal{A}\left(K_{\infty}\right) \subset V\left(K_{\infty}\right)$ и (неканонически)

$$
V\left(K_{\infty}\right) / \mathcal{A}\left(K_{\infty}\right) \cong Y_{\infty}
$$

(см. $[11,(1.10)])$. Пусть $\kappa: G_{\infty} \rightarrow \mathbf{Z}_{\ell}^{*}-$ круговой характер, определенный формулой $\zeta_{n}^{\sigma}=\zeta_{n}^{\kappa(\sigma)}$ для $n=1,2, \ldots$ Мы определим автоморфизм $w: R_{\infty} \rightarrow R_{\infty}$ формулой $w(\sigma)=\kappa(\sigma) \sigma^{-1}$ для любого $\sigma \in G_{\infty}$.

Теорема С [11, теорема 4.1]. При заданном поле $K_{\infty}$ имеют место равенства

$$
C_{\infty}=w\left(S_{\infty}\right) V\left(K_{\infty}\right), \quad P_{\infty}=w\left(S_{\infty}^{\prime}\right) V\left(K_{\infty}\right)
$$

Периодические $\Lambda$-модули $V\left(K_{\infty}\right)^{+} / C_{\infty}$ и $R_{\infty}^{+} / w\left(S_{\infty}\right)$ изоморфньк как $R_{\infty}$-модули.

Хорошо известно (и без труда может быть получено из [15, лемма 2.1]), что характеристические ряды $\Lambda$-периодического $R_{\infty}$-модуля $R_{\infty}^{-} / S_{\infty}$ совпадают с рядами Ивасавы для нечетных характеров $\chi, \chi \neq \omega$, и совпадают с $\eta(T, \omega)$ для $\chi=\omega$. Согласно теореме Ферреро-Вашингтона модуль $R_{\infty}^{-} / S_{\infty}$ конечно порожден над $Z_{\ell}$. Следовательно, $R_{\infty}^{-} / S_{\infty}$ имеет тривиальные ряды Ивасавы для четных характеров $\chi$. Таким образом, $V\left(K_{\infty}\right)^{+} / C_{\infty}$ имеет тривиальные характеристические ряды для нечетных характеров $\chi$. Поскольку $w(\gamma)=\kappa \gamma^{-1}$, мы получаем, что $w(T)=\frac{\kappa}{1+T}-1$. Автоморфизм $w$ отображает $\left(R_{\infty}^{-} / S_{\infty}\right)_{\chi}$ на $\left(R_{\infty}^{+} / w\left(S_{\infty}\right)\right)_{\bar{\chi} \omega}$. Поэтому согласно теореме $\mathrm{C}$ характеристический ряд модуля $V\left(K_{\infty}\right)^{+} / C_{\infty}$, соответствующий четному характеру $\theta$, совпадает с $f\left(\frac{\kappa}{1+T}-1, \bar{\theta} \omega\right)$ при $\theta \neq 1$ (соответственно с $\eta\left(\frac{\kappa}{1+T}-1, \omega\right)$ при $\left.\theta=1\right)$. Тогда, используя $(2.13)$ и $(2.3)$, мы получаем формулу (2.11). Чтобы получить (2.12), достаточно заметить, что $\theta$-компонента $Y_{\infty, \theta}$ конечна при $\theta=1$. Теорема доказана. 


\section{§3. Вычисление некоторых характеристических рядов}

Пусть $K_{\infty}$ имеет тот же смысл, что и в $\S 2$. Пусть $\bar{M}_{\infty}-$ максимальное абелево неразветвленное $\ell$-расширение поля $K_{\infty}$ и $M_{\infty}$ - максимальное подрасширение в $\bar{M}_{\infty} / K_{\infty}$ такое, что $\ell$ полностью распадается в $M_{\infty} / K_{\infty}$. Положим $\bar{T}_{\ell}\left(K_{\infty}\right):=$ $G\left(\bar{M}_{\infty} / K_{\infty}\right)$ и $T_{\ell}\left(K_{\infty}\right):=G\left(M_{\infty} / K_{\infty}\right)$. Пусть $R_{\ell}\left(K_{\infty}\right)$ - ядро естественного эпиморфизма $\bar{T}_{\ell}\left(K_{\infty}\right) \rightarrow T_{\ell}\left(K_{\infty}\right)$. Таким образом, группа $R_{\ell}\left(K_{\infty}\right)=G\left(\bar{M}_{\infty} / M_{\infty}\right)$ совпадает с подгруппой групшы $\bar{T}_{\ell}\left(K_{\infty}\right)$, порожденной подгруппами разложения для всех точек, лежащих над $\ell$. Отметим, что $\bar{T}_{\ell}\left(K_{\infty}\right), T_{\ell}\left(K_{\infty}\right)$ и $R_{\ell}\left(K_{\infty}\right)$ имеют естественную структуру $R_{\infty}$-модулей.

Следующая теорема, известная как "основная гипотеза теории Ивасавы", дает нам характеристические ряды модуля $\bar{T}_{\ell}\left(K_{\infty}\right)$, соответствующие нечетным характерам $\chi$ первого рода, или, что эквивалентно, характеристические ряды модуля $\bar{T}_{\ell}\left(K_{\infty}\right)^{-}$для всех $\chi$ первого рода.

ТеоремА D. Для любого нечетного характера первого рода $\chi \neq \omega$ соответствующий ему характеристический ряд модуля $\bar{T}_{\ell}\left(K_{\infty}\right)$ совпадает с рядом Ивасавь $f(T, \chi)$. Для $\chi=\omega$ характеристический ряд модуля $\bar{T}_{\ell}\left(K_{\infty}\right)$ для $\chi=\omega$ совпадает с $\eta(T, \omega)$ и, следовательно, обратим.

Эта теорема была доказана в [13] (для нечетных $\ell$ ) и в [17] (для $\ell=2$ ). Другое доказательство, основанное на эйлеровых системах Кольвагина, было дано в [4] (см. также [6], [12], [14]). Положим $\mathbf{Z}_{\ell}(1)=\underset{\lim }{\longleftarrow} \mu_{\ell}\left(K_{n}\right)$. Для любого $R_{\infty \text {-мо- }}$ дуля $A$, конечно порожденного над $\mathbf{Z}_{\ell}$, мы превратим группу $\operatorname{Hom}_{\mathbf{Z}_{\ell}}\left(A, \mathbf{Z}_{\ell}(1)\right)$ в $R_{\infty}$-модуль, полагая $(\sigma \chi)(a)=\kappa(\sigma) \chi\left(\sigma^{-1}(a)\right)$ для любых $\sigma \in G_{\infty}, \chi \in$ $\operatorname{Hom}_{\mathbf{Z}_{\ell}}\left(A, \mathbf{Z}_{\ell}(1)\right), a \in A$.

ПРЕДЛОЖЕНИЕ 3.1. Для всех характеров характеристические $R_{\infty}$-модулей $\mathcal{A}_{\infty}^{+} / C_{\infty}$ u $\operatorname{Hom}_{\mathbf{Z}_{\ell}}\left(T_{\ell}\left(K_{\infty}\right)^{-}, \mathbf{Z}_{\ell}(1)\right)$ совпадают.

ДоказАтельство. Для произвольного нечетного характера первого рода $\chi$ положим $\theta=\bar{\chi} \omega$. Тогда $R_{\infty}$-модули $\operatorname{Hom}_{\mathbf{z}_{\ell}}\left(\left(\bar{T}_{\ell}\left(K_{\infty}\right)^{-}\right)_{\chi}, \mathbf{Z}_{\ell}(1)\right)$ и $\left(\operatorname{Hom}_{\mathbf{Z}_{\ell}}\left(\bar{T}_{\ell}\left(K_{\infty}\right)^{-}, \mathbf{Z}_{\ell}(1)\right)\right)_{\theta}$ квазиизоморфны и, следовательно, имеют одинаковые характеристические ряды. Согласно теореме D модуль $\left(\bar{T}_{\ell}\left(K_{\infty}\right)^{-}\right)_{\chi}=$ $\bar{T}_{\ell}\left(K_{\infty}\right)_{\chi}$ имеет единственный нетривиальный характеристический ряд $f(T, \chi)$, соответствующий характеру $\chi(\eta(T, \omega)$ при $\chi=\omega)$. Если $\alpha_{1}, \ldots, \alpha_{r}-$ нули этого ряда, то $1+\alpha_{1}, \ldots, 1+\alpha_{r}-$ собственные значения оператора $\gamma$, действующего на $\bar{T}_{\ell}\left(K_{\infty}\right)_{\chi} \otimes_{\mathcal{O}_{\chi}} L_{\chi}$, где $L_{\chi}-$ поле частных кольца $\mathcal{O}_{\chi}$. Тогда $\gamma$, рассматриваемьй как оператор на $L_{\chi}$-пространстве $\left(\operatorname{Hom}_{\mathbf{z}_{\ell}}\left(\bar{T}_{\ell}\left(K_{\infty}\right)^{-}, \mathbf{Z}_{\ell}(1)\right)\right)_{\theta} \otimes_{\mathcal{O}_{\chi}} L_{\chi}$, имеет собственные значения $\beta_{1}=\kappa\left(1+\alpha_{1}\right)^{-1}, \ldots, \beta_{r}=\kappa\left(1+\alpha_{r}\right)^{-1}$. Прямая проверка показьвает, что $\beta_{1}-1, \ldots, \beta_{r}-1$ - это все нули ряда

$$
\begin{aligned}
& g(T, \theta)=f\left(\frac{\kappa}{1+T}-1, \bar{\theta} \omega\right) \text { при } \theta \neq 1, \\
& g(T, \theta)=\eta\left(\frac{\kappa}{1+T}-1, \bar{\theta} \omega\right) \text { при } \theta=\omega,
\end{aligned}
$$

удовлетворяюшие условию $\nu_{\ell}\left(\beta_{i}-1\right)>0$ для $i=1, \ldots, r$. Следовательно, $\beta_{1}-1, \ldots, \beta_{r}-1$ - это все корни отмеченного многочлена, ассоциированного с рядом $g(T, \theta)$. Поэтому ряд $g(T, \theta)$ является характеристическим рядом модуля $\operatorname{Hom}_{\mathbf{z}_{\ell}}\left(\bar{T}_{\ell}\left(K_{\infty}\right)^{-}, \mathbf{Z}_{\ell}(1)\right)$ для любого четного характера первого рода $\theta$. 
Рассмотрим теперь естественную последовательность отображений

$$
0 \longrightarrow R_{\ell}\left(K_{\infty}\right)^{-} \longrightarrow \bar{T}_{\ell}\left(K_{\infty}\right)^{-} \longrightarrow T_{\ell}\left(K_{\infty}\right)^{-} \longrightarrow 0
$$

Последовательность (3.3) точна по модулю конечных групп. Пусть $\mathcal{D}_{\infty}-R_{\infty}$-модуль из доказательства предложения 2.4. Тогда $\mathcal{D} \infty \cong \mathbf{Z}_{\ell}\left[G_{\infty} / G_{\infty, v}\right]$ как $R_{\infty}$-модуль, где $G_{\infty, v}$ - подгруппа разложения любой точки $v \mid \ell$ в группе $G_{\infty}$. Мы имеем естественный эпиморфизм $f: \mathcal{D}_{\infty} \rightarrow R_{\ell}\left(K_{\infty}\right)$, который переводит любую точку $v \mid \ell$ в автоморфизм $\Phi$ робениуса в ее подгруппе разложения в группе $\bar{T}_{\ell}\left(K_{\infty}\right)$. Отображение $f$ индуцирует отображение $f^{-}: \mathcal{D}_{\infty}^{-} \longrightarrow R_{\ell}\left(K_{\infty}\right)^{-}$, которое, как хорошо известно, является квазиизоморфизмом (и даже изоморфизмом при $\ell \neq 2$ ). Из явного вида модулей $\mathcal{D}_{\infty}$ и $Y_{\infty}$ следует, что

$$
\operatorname{Hom}_{\mathbf{z}_{\ell}}\left(\mathcal{D}_{\infty}, \mathbf{Z}_{\ell}(1)\right) \cong Y_{\infty} \quad(\text { неканонически })
$$

Таким образом, имеется квазиизоморфизм

$$
\operatorname{Hom}_{\mathbf{z}_{\ell}}\left(\mathcal{D}_{\infty}^{-}, \mathbf{Z}_{\ell}(1)\right) \cong Y_{\infty}^{+}
$$

Сопоставляя теорему 2.1 с формулами (3.1)-(3.4) и учитывая, что модуль $\left(Y_{\infty}\right) \theta$ конечен для $\theta=1$, мы получаем утверждение нашего предложения.

Для любого поля $K_{n}$ и его группы единищ $U\left(K_{n}\right)$ положим $\bar{U}\left(K_{n}\right):=U\left(K_{n}\right) /$ $\mu\left(K_{n}\right)$ и $U_{\infty}=\underline{\lim }\left(\bar{U}\left(K_{n}\right)[\ell]\right)$, где проективный предел берется относительно норменных отображений. Имеются естественные включения $C_{\infty} \subseteq U_{\infty} \subseteq \mathcal{A}_{\infty}^{+}$, поэтому существует точная последовательность

$$
0 \longrightarrow U_{\infty} / C_{\infty} \longrightarrow \mathcal{A}_{\infty}^{+} / C_{\infty} \longrightarrow \mathcal{A}_{\infty}^{+} / U_{\infty} \longrightarrow 0
$$

Пусть $U_{S}\left(K_{n}\right)$ - группа $S$-единищ поля $K_{n}$, где $S$ - множество всех точек над $\ell$ в поле $K_{n}$. Положим $\bar{U}_{S}\left(K_{n}\right):=U_{S}\left(K_{n}\right) / \mu\left(K_{n}\right)$ и $U_{S, \infty}:=\lim \bar{U}_{S}\left(K_{n}\right)[\ell]$, где проективный предел берется относительно норменных отображений. Тогда имеется естественное включение $U_{S, \infty} \subseteq H\left(K_{\infty}\right)^{+}$. Если $k-$ произвольное вещественное абелево поле и $k_{\infty}$ - круговое $\mathbf{Z}_{\ell}$-расширение $k$, то можно аналогичным образом определить абелевы про- $\ell$-группы $H\left(k_{\infty}\right), \mathcal{A}\left(k_{\infty}\right), U\left(k_{\infty}\right)$ и $U_{S}\left(k_{\infty}\right)$. Если $k_{\infty} \subseteq K_{\infty}$ для некоторого кругового поля $K=K_{0}$, то все эти группы можно рассмативать как $R_{\infty}$-модули.

ПРЕДЛОЖЕНИЕ $3.2 . R_{\infty}$-модули $\mathcal{A}_{\infty}^{+} / U_{\infty}$ u $H_{\infty}^{+} / U_{S, \infty}$ квазиизоморфны. Пусть $k$ - произвольное вещественное абелево поле, $k_{\infty}-$ круговое $\mathbf{Z}_{\ell}$-расширение поля $k$, причем $k_{\infty} \subseteq K_{\infty}$ для некоторого кругового поля $K$. Тогда $R_{\infty}$-модули $\mathcal{A}\left(k_{\infty}\right) / U\left(k_{\infty}\right)$ и $H\left(k_{\infty}\right) / U_{S}\left(k_{\infty}\right)$ квазиизоморфньи.

ДоказАтельство. Так как $U_{\infty}=U_{S, \infty} \cap \mathcal{A}_{\infty}^{+}$, естественное отображение

$$
i: \mathcal{A}_{\infty}^{+} / U_{\infty} \longrightarrow H_{\infty}^{+} / U_{S, \infty}
$$

является вложением. Поэтому достаточно проверить, что $i$ имеет конечное коядро. Для этого мы дадим интерпретацию рассматриваемых групп в терминах теории полей классов. Пусть $N_{\infty}$ - максимальное $\ell$-разветвленное абелево $\ell$-расширение 
поля $K_{\infty}$. Положим $X_{\infty}:=G\left(N_{\infty} / K_{\infty}\right)$. Тогда имеются естественные точные последовательности $R_{\infty}$-модулей

$$
\begin{aligned}
& 0 \longrightarrow W_{\infty} \longrightarrow X_{\infty} \longrightarrow T_{\ell}\left(K_{\infty}\right) \longrightarrow 0 \\
& 0 \longrightarrow \bar{W}_{\infty} \longrightarrow X_{\infty} \longrightarrow \bar{T}_{\ell}\left(K_{\infty}\right) \longrightarrow 0
\end{aligned}
$$

где $W_{\infty}$ (соответственно $\left.\bar{W}_{\infty}\right)$ означает подгруппу группы $X_{\infty}$, порожденную подгруппами разложения (соответственно подгруппами инерции) для всех точек $v \mid \ell$ поля $K_{\infty}$. Пусть $f: Y_{\infty} \rightarrow X_{\infty}$ - естественное отображение, возникаюшее из теории полей классов. Тогда $\operatorname{Im} f \subset \bar{W}_{\infty} \subseteq W_{\infty}$ и из теории полей классов следует, что

$$
W_{\infty} / \operatorname{Im} f \cong H_{\infty} / U_{S, \infty}, \quad \bar{W}_{\infty} / \operatorname{Im} f \cong \mathcal{A}_{\infty} / U_{\infty}
$$

Таким образом,

$$
W_{\infty} / \bar{W}_{\infty}=R_{\ell}\left(K_{\infty}\right) \cong H_{\infty} /\left(\mathcal{A}_{\infty} \cdot U_{S, \infty}\right) \supset \text { Coker } i=H_{\infty}^{+} / \mathcal{A}_{\infty}^{+} \cdot U_{S, \infty}
$$

Следовательно, Coker $i \subset R_{\ell}\left(K_{\infty}\right)^{+}$. Отметим, что группа $R_{\ell}\left(K_{\infty}\right)^{+}$конечно порождена. С другой стороны, согласно гипотезе Леопольдта над полем $K_{n}^{+}$не сушествует некруговых $\mathbf{Z}_{\ell}$-расширений ни при каких $n$. Значит, $R_{\ell}\left(K_{\infty}\right)^{+}{ }_{-}$периодический модуль и, следовательно, он конечен. Итак, группа Coker $i$ конечна, следовательно, $i$ является квазиизоморфизмом. Аналогичным образом устанавливается квазиизоморфизм модулей $\mathcal{A}\left(k_{\infty}\right) / U\left(k_{\infty}\right)$ и $H\left(k_{\infty}\right) / U_{S}\left(k_{\infty}\right)$. Предложение доказано.

ТЕорема 3.1. $R_{\infty}$-модули $T_{\ell}\left(K_{\infty}\right)^{+}$и $U_{\infty} / C_{\infty}$ имеют одинаковые характеристические ряды (для любого четного характера первого рода $\theta$ ).

ДокАЗАТЕльство. Мы выведем эту теорему из некоторых теорем двойственности, полученных в [10]. Положим $\bar{X}_{\infty}:=X_{\infty} / f\left(Y_{\infty}\right)$ и $\mathcal{A}_{\ell}(K)=\bar{X}_{\infty} / H_{\infty}^{-}$, где $X_{\infty}, Y_{\infty}$ имеют тот же смысл, что и в доказательстве предложения 3.2 . Тогда справедлива

Теорема Е. $R_{\infty}$-модули $2 \mathcal{A}_{\ell}(K)$ u $\operatorname{Hom}_{\mathbf{Z}_{\ell}}\left(2 \mathcal{A}_{\ell}(K), \mathbf{Z}_{\ell}(1)\right)$ квазиизоморфньи.

Действительно, если $\ell \neq 2$, то из теоремы Ферреро-Вашингтона следует, что модуль $\mathcal{A}_{\ell}(K)$ конечно порожден над $\mathbf{Z}_{\ell}$. Тогда из [10, следствие из теоремы 4.1] вытекает, что $\mathcal{A}_{\ell}(K) \cong \operatorname{Hom}_{\mathbf{z}_{\ell}}\left(\mathcal{A}_{\ell}(K), \mathbf{Z}_{\ell}(1)\right)$. Хотя случай $\ell=2$ не был явно рассмотрен в [10], в этой работе было отмечено, что все ее результаты остаются справедливыми в случае $\ell=2$ с точностью до подгрупп и факторгрупп периода 2 . Если $\ell=2$, то модуль $2 \mathcal{A}_{\ell}(K)$ конечно порожден над $\mathbf{Z}_{\ell}$, поэтому, повторяя доказательство теоремы 4.1 работы [10] для этого случая, мы получаем утверждение теоремы Е.

Из теоремы Е следует существование квазиизоморфизма

$$
2 \mathcal{A}_{\ell}(K)^{+} \sim \operatorname{Hom}_{\mathbf{z}_{\ell}}\left(2 \mathcal{A}_{\ell}(K)^{-}, \mathbf{z}_{\ell}(1)\right)
$$

Определение модуля $\mathcal{A}_{\ell}(K)$ и формула (3.6) показывают, что

$$
2 \mathcal{A}_{\ell}(K)^{-} \sim T_{\ell}\left(K_{\infty}\right)^{-} .
$$

Следовательно, модуль $2 \mathcal{A}_{\ell}(K)^{+}$входит в точную последовательность

$$
0 \longrightarrow 2 H_{\infty} /\left(2 H_{\infty} \cap\left(H_{\infty}^{-} \cdot U_{S, \infty}\right)\right) \stackrel{\alpha}{\longrightarrow} 2 \mathcal{A}_{\ell}(K)^{+} \stackrel{\beta}{\longrightarrow} 2 T_{\ell}\left(K_{\infty}\right)^{+}
$$


с конечной группой coker $\beta$.

Из предложения 3.1 и формул $(3.8),(3.9)$ следует, что модули $2 \mathcal{A}_{\ell}(K)^{+}$и $\mathcal{A}_{\infty}^{+} / C_{\infty}$ имеют одинаковые характеристические ряды. Тогда из предложения 3.2 вытекает, что

$$
2 H_{\infty} /\left(2 H_{\infty} \cap\left(H_{\infty}^{-} \cdot U_{S, \infty}\right)\right) \sim H_{\infty}^{-} / U_{S, \infty} \sim \mathcal{A}_{\infty}^{+} / U_{\infty}
$$

Поэтому из $(3.5),(3.10)$ и из предложения 2.1 следует, что $U_{\infty} / C_{\infty}$ и $T_{\ell}\left(K_{\infty}\right)^{+}$ имеют одинаковые характеристические ряды. Теорема доказана.

Пусть теперь $k$ - произвольное вещественное абелево поле, $k_{\infty}$ - круговое

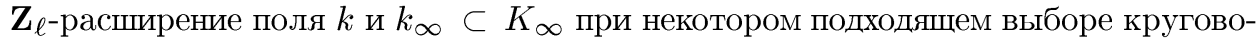
го поля $K=K_{0}$.

Теорема 3.2. Пусть $k_{\infty}$ и $K_{\infty}$ имеют тот же смисл, что и выше. Положим $H=G\left(K_{\infty} / k_{\infty}\right)$. Тогда $R_{\infty}$-модули $T_{\ell}\left(k_{\infty}\right)$ и $U_{\infty}^{H} / C_{\infty}^{H}$ имеют одинаковые характеристические ряды.

ДокАЗАТЕльство. Существуют квазиизоморфизмы $T_{\ell}\left(k_{\infty}\right) \sim T_{\ell}\left(K_{\infty}\right)^{H}$, $U_{\infty}^{H} / C_{\infty}^{H} \sim\left(U_{\infty} / C_{\infty}\right)^{H}$, поэтому наша теорема непосредственно следует из теоремы 3.1.

\section{§4. Доказательство теоремы 3}

Пусть $k, k_{\infty}, K_{\infty}, R_{\infty}$ и $H=G\left(K_{\infty} / k_{\infty}\right)$ имеют тот же смысл, что и в $\S 3$. Напомним, что мы определили $R_{\infty}$-модули $H\left(k_{\infty}\right), U_{S}\left(k_{\infty}\right)$, остаюшиеся неподвижными под действием $H$. Положим $\widetilde{H}\left(k_{\infty}\right):=\left(H_{\infty}\right)^{H}, \widetilde{U}_{S}\left(k_{\infty}\right):=\left(U_{S, \infty}\right)^{H}$, $\widetilde{C}\left(k_{\infty}\right):=\left(C_{\infty}\right)^{H}$. Тогда в силу предложения 1.1 из [8] $R_{\infty}$-модули $\widetilde{H}\left(k_{\infty}\right)$, $\widetilde{U}_{S}\left(k_{\infty}\right), \widetilde{C}\left(k_{\infty}\right)$, являются $\Lambda$-свободными. Пусть $H(k)$ (соответственно $\left.\widetilde{H}(k)\right)$ образ естественной проекции $H\left(k_{\infty}\right) \rightarrow \mathcal{B}(k)$ (соответственно $\widetilde{H}\left(k_{\infty}\right) \rightarrow$ $\left.\mathcal{B}(k) \otimes_{\ell} \mathbf{Q}_{\ell}\right)$. Пусть $U_{S}^{*}(k)$ (соответственно $\left.\widetilde{U}_{S}^{*}(k)\right)$ - образ естественной проекции $U_{S}\left(k_{\infty}\right) \rightarrow H(k)\left(\right.$ соответственно $\left.\widetilde{U}_{S}\left(k_{\infty}\right) \rightarrow \widetilde{H}(k)\right)$. Положим $\widehat{U}_{S}(k):=\bar{U}_{S}(k)[\ell] \cap$ $H(k)$. Таким образом, $\widehat{U}_{S}(k)$ совпадает с подгруппой всех элементов групшы $\bar{U}_{S}(k)[\ell]$, являющихся локальными универсальными нормами из поля $k_{\infty}$. Положим

$$
\begin{aligned}
& \widetilde{C}_{S}\left(k_{\infty}\right):=\left\{x \in \widetilde{H}\left(k_{\infty}\right) \mid\left(\gamma_{n}-1\right) x \in \widetilde{C}\left(k_{\infty}\right) \text { при некотором } n\right\} \\
& C_{S}\left(k_{\infty}\right):=\widetilde{C}_{S}\left(k_{\infty}\right) \cap U_{S}\left(k_{\infty}\right) .
\end{aligned}
$$

Из определения и из предложения 3.2 следует, что модули $\widetilde{H}\left(k_{\infty}\right) / \widetilde{C}_{S}\left(k_{\infty}\right)$ и $\left(\mathcal{A}_{\infty}^{+} / C_{\infty}\right)^{H}$ квазиизоморфны.

Пусть $\widetilde{C}_{S}(k)$ (соответственно $\left.C_{S}(k)\right)$ - образ естественной проекции $\widetilde{C}_{S}\left(k_{\infty}\right) \rightarrow$ $\widetilde{H}(k)$ (соответственно $C_{S}\left(k_{\infty}\right) \rightarrow H(k)$ ). Мы будем называть группу $C_{S}(k)$ груnnой круговых $S$-единии. Следуюшее предложение выясняет связь между всеми этими группами.

ПРЕДЛОЖЕНИЕ 4.1. Существуют естественные включения

$$
\widetilde{H}(k) \supseteq \widetilde{U}_{S}^{*}(k) \supseteq \widetilde{C}_{S}(k), \quad H(k) \supseteq \widehat{U}_{S}(k) \supseteq U_{S}^{*}(k) \supseteq C_{S}(k),
$$

причем все индексы, возникающие в (4.1), конечнь. 
Eсли $\ell \neq 2$, mо $H(k)=\widetilde{H}\left(k_{\infty}\right), \quad U_{S}\left(k_{\infty}\right)=\widetilde{U}_{S}\left(k_{\infty}\right), \quad C_{S}\left(k_{\infty}\right)=\widetilde{C}_{S}\left(k_{\infty}\right)$, $H(k)=\widetilde{H}(k), \quad U_{S}^{*}(k)=\widetilde{U}_{S}^{*}(k) u C_{S}(k)=\widetilde{C}_{S}(k)$.

Если $\ell=2 u \sqrt{-1} \in k_{\infty, v}$ для некоторой точки $v \mid \ell$ (следовательно, для любой точки $v \mid \ell)$, то $H\left(k_{\infty}\right)=\widetilde{H}\left(k_{\infty}\right), \quad H(k)=\widetilde{H}(k)$.

Eсли $\ell=2 u \sqrt{-1} \notin k_{\infty, v}$ для $v \mid \ell, m o$

$$
\widetilde{H}\left(k_{\infty}\right) / H\left(k_{\infty}\right) \cong \prod_{v \mid \ell} \mu_{\ell}\left(k_{\infty, v}\right) \cong(\mathbf{Z} / 2 \mathbf{Z})^{r},
$$

где $r$ - число точек $v \mid \ell$ в поле $k_{\infty}$.

Если $\ell=2$, то в любом из случаев

$$
\begin{aligned}
{\left[\widetilde{U}_{S}\left(k_{\infty}\right): U_{S}\left(k_{\infty}\right)\right] } & =\left[\widetilde{C}_{S}\left(k_{\infty}\right): C_{S}\left(k_{\infty}\right)\right] \\
=\left[\widetilde{U}_{S}^{*}(k): U_{S}^{*}(k)\right] & =\left[\widetilde{C}_{S}(k): C_{S}(k)\right]=2, \\
\widetilde{U}_{S}(k) / \widetilde{C}_{S}(k) & =U_{S}^{*}(k) / C_{S}(k) .
\end{aligned}
$$

ДОКАЗАТЕЛЬСТВО. Если $\bar{\Gamma}=G\left(k_{\infty} / k\right)$, то для любого достаточно большого $n$ естественная проекция $G_{\infty} \rightarrow G\left(k_{\infty} / \mathbf{Q}\right)$ отображает $\Gamma_{n}$ на $\bar{\Gamma}_{n-s}$, где $s \geqslant 0$ не зависит от $n$. Естественное включение $\widetilde{H}\left(k_{\infty}\right) / \widetilde{U}_{S}\left(k_{\infty}\right) \subseteq H_{\infty}^{+} / U_{S, \infty}$ индуцирует для всех достаточно больших $n$ включение

$$
\left(\widetilde{H}\left(k_{\infty}\right) / \widetilde{U}_{S}\left(k_{\infty}\right)\right)^{\bar{\Gamma}_{n-s}} \subseteq\left(H_{\infty}^{+} / U_{S, \infty}\right)^{\Gamma_{n}} .
$$

Согласно [9, лемма 7.2 и теорема 7.2] $\Lambda$-модули $H_{\infty}$ и $U_{S, \infty}$ свободны. Следовательно, $H_{\infty}^{+}$свободен как $\Lambda$-модуль, и $H_{\infty}^{+} / U_{S, \infty}$ не содержит нетривиальных конечных подмодулей. С другой стороны, модуль $\left(H_{\infty}^{+} / U_{S, \infty}\right)^{\Gamma_{n}}$ не содержит $\Lambda$-подмодулей, которые изоморфны как $\Lambda$-модули модулю $\mathbf{Z}_{\ell}$, поскольку в нашем случае справедлива гипотеза Леопольдта. Таким образом, $\left(H_{\infty}^{+} / U_{S, \infty}\right)^{\Gamma_{n}}=0$ и $\left(\widetilde{H}\left(k_{\infty}\right) / \widetilde{U}_{S}\left(k_{\infty}\right)\right)^{\bar{\Gamma}_{n}}=0$ для всех достаточно больших $n$. Из определения модуля $\widetilde{C}_{S}\left(k_{\infty}\right)$ и из последнего равенства следует, что $\widetilde{C}_{S}\left(k_{\infty}\right) \subseteq \widetilde{U}_{S}\left(k_{\infty}\right)$. Поэтому $\widetilde{C}_{S}\left(k_{\infty}\right) \subseteq \widetilde{U}_{S}\left(k_{\infty}\right)$ и $\widetilde{C}_{S}(k) \subseteq \widetilde{U}_{S}^{*}(k), C_{S}(k) \subseteq U_{S}^{*}(k)$. Остальные включения в (4.1) очевидны.

Согласно теореме 1.1 модуль $H_{\infty}^{+} / C_{\infty}$ является $\Lambda$-периодическим, следовательно, $\widetilde{H}\left(k_{\infty}\right) / \widetilde{C}_{S}\left(k_{\infty}\right)$ является $\bar{\Gamma}$-периодическим, причем из определения модуля $\widetilde{C}_{S}\left(k_{\infty}\right)$ следует, что $\left(\widetilde{H}\left(k_{\infty}\right) / \widetilde{C}_{S}\left(k_{\infty}\right)\right)^{\bar{\Gamma}_{n}}=0$ при любом $n$. Поскольку $\widetilde{H}\left(k_{\infty}\right)$ и $\widetilde{C}_{S}\left(k_{\infty}\right)$ свободны как $\bar{\Gamma}$-модули, мы имеем естественную точную последовательность (см. $[9,(1.2)])$

$$
\begin{aligned}
0= & \left(\widetilde{H}\left(k_{\infty}\right) / \widetilde{C}_{S}\left(k_{\infty}\right)\right)^{\bar{\Gamma}} \longrightarrow \widetilde{C}_{S}\left(k_{\infty}\right)_{(0)} \\
& \longrightarrow \widetilde{H}\left(k_{\infty}\right)_{(0)} \longrightarrow\left(\widetilde{H}\left(k_{\infty}\right) / \widetilde{C}_{S}\left(k_{\infty}\right)\right)_{(0)} \longrightarrow 0,
\end{aligned}
$$

где $A_{(0)}=A /(\bar{\gamma}-1) A$ для любого $\bar{\Gamma}$-модуля $A$.

Из локальной теории полей классов следует, что $\widetilde{H}\left(k_{\infty}\right)_{(0)} \cong \widetilde{H}(k)$. Поэтому (4.4) показывает, что $\widetilde{C}_{S}\left(k_{\infty}\right)_{(0)} \cong \widetilde{C}_{S}(k)$ и

$$
\left(\widetilde{H}\left(k_{\infty}\right) / \widetilde{C}_{S}\left(k_{\infty}\right)\right)_{(0)} \cong \widetilde{H}(k) / \widetilde{C}_{S}(k) .
$$


Так как $\widetilde{H}\left(k_{\infty}\right)$ и $\widetilde{C}_{S}\left(k_{\infty}\right)$ являются свободными $\bar{\Gamma}$-модулями одинакового ранга,

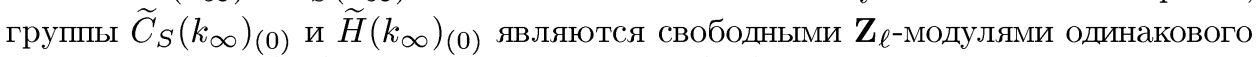
ранга. Тогда из (4.4) следует, что группы в (4.5) конечны. Из этого, а также из равенств $C_{S}(k)=\widetilde{C}_{S}(k)$ при $\ell \neq 2,\left[\widetilde{C}_{S}(k): C_{S}(k)\right]=2$ при $\ell=2$, которые будут доказаны ниже, вытекает конечность всех индексов в (4.1).

Если $\ell \neq 2$, то согласно [9, лемма 7.2] мы получаем, что $H_{\infty}$ является свободным $\Gamma$-модулем, а $H\left(k_{\infty}\right)$ является свободным $\bar{\Gamma}$-модулем. Рассмотрим включения $\widetilde{H}\left(k_{\infty}\right) \supseteq H\left(k_{\infty}\right) \supseteq N_{H}\left(H_{\infty}\right)$, где $H=G\left(K_{\infty} / k_{\infty}\right)$. Из локальной теории полей классов следует, что группа когомологий Тэйта $H^{0}\left(H, H_{\infty}\right)$ конечна и, следовательно, $\left[\widetilde{H}\left(k_{\infty}\right): H\left(k_{\infty}\right)\right]<\infty$. Поскольку оба рассматриваемых модуля свободны над $\bar{\Gamma}$, мы получаем, что $\widetilde{H}\left(k_{\infty}\right)=H\left(k_{\infty}\right)$.

Равенство $\widetilde{U}_{S}\left(k_{\infty}\right)=U_{S}\left(k_{\infty}\right)$ может быть доказано тем же путем. Из [9, теорема 7.2] следует, что $U_{S, \infty}$ является свободным $\Gamma$-модулем, а $U_{S}\left(k_{\infty}\right)$ является свободным $\bar{\Gamma}$-модулем. Порядки групп когомологий Тэйта $H^{0}\left(H, \bar{U}_{S}\left(K_{n}\right)\right)$ могут быть оценены сверху в терминах числа образующих $\ell$-групп классов $\mathrm{Cl}\left(K_{n}\right)$. Поэтому конечность групшы $H^{0}\left(H, U_{S, \infty}\right)$ следует из тривиальности $\mu$-инварианта Ивасавы поля $K_{\infty}$. Тогда мы получаем $\widetilde{C}_{S}\left(k_{\infty}\right)=C_{S}\left(k_{\infty}\right), \widetilde{H}(k)=H(k)$, $\widetilde{U}_{S}^{*}(k)=U_{S}^{*}(k)$. Это доказывает наше предложение при $\ell \neq 2$.

Если $\ell=2$ и $\sqrt{-1} \in k_{\infty, v}$ для любой точки $v \mid \ell$, то, применяя лемму 7.2 из [9], мы снова получаем, что $H\left(k_{\infty}\right)$ является свободным $\bar{\Gamma}$-модулем и $\widetilde{H}\left(k_{\infty}\right)=H\left(k_{\infty}\right)$.

В случае, когда $\ell=2$ и $\sqrt{-1} \notin k_{\infty, v}$ для любой точки $v \mid \ell$, лемма 7.2 работы [9] нуждается в некоторых исправлениях. Правильное утверждение, которое легко можно доказать, используя локальную теорию полей классов, говорит, что в этом случае $H\left(k_{\infty}\right)$ является $\bar{\Gamma}$-модулем без кручения и сушествует вложение $H\left(k_{\infty}\right) \hookrightarrow F$, где $F-$ свободный $\bar{\Gamma}$-модуль, причем $F / H\left(k_{\infty}\right) \cong \prod_{v \mid \ell} \mu_{\ell}\left(k_{\infty, v}\right)$ как модуль Галуа (заметим, что $\mu_{\ell}\left(k_{\infty, v}\right)=\{ \pm 1\}$ для любой точки $\left.v \mid \ell\right)$.

Если $\ell=2$ и $\sqrt{-1} \notin k_{\infty}$ (именно этот случай имеет место для вещественного абелева $k$ ), то теорема 7.2 работы [9] также нуждается в исправлениях. В этом случае правильное утверждение состоит в том, что модуль $U_{S}\left(k_{\infty}\right)$ изоморфен подмодулю индекса 2 некоторого свободного $\bar{\Gamma}$-модуля. Действительно, теорема 7.2 вместе с доказательством, приведенным в [9], остаются справедливыми для поля $k_{\infty}^{\prime}=k_{\infty}(\sqrt{-1})$. Следовательно, $U_{S}\left(k_{\infty}^{\prime}\right)$ является свободным $\bar{\Gamma}$-модулем. Из теории Куммера следует, что $\left[U_{S}\left(k_{\infty}^{\prime}\right): U_{S}\left(k_{\infty}\right)\right] \leqslant 2$. С другой стороны, модуль $U_{S}\left(k_{\infty}\right)$ не является $\bar{\Gamma}$-свободным. Последнее утверждение вытекает из $[9$, предложение 1.2] и из того наблюдения, что -1 содержится в образе естественной проекции $U_{S}\left(k_{\infty}\right) \rightarrow U_{S}\left(k_{n}\right)[\ell]$ (достаточно проверить это для поля $k=\mathbf{Q}$ ). Таким образом, мы получаем $\left[U_{S}\left(k_{\infty}^{\prime}\right): U_{S}\left(k_{\infty}\right)\right]=2$, как и требовалось. Более того, мы получаем, что $\widetilde{U}_{S}\left(k_{\infty}\right)=U_{S}\left(k_{\infty}^{\prime}\right)$.

Рассмотрим теперь круговое число $\varepsilon_{\infty}(1)=\underline{\lim }\left(1-\zeta_{n}\right) \in U_{S}\left(k_{\infty}^{\prime}\right)$. Как было доказано Синноттом (см. [15, раздел 1]), мы имеем при любом $n$

$$
1-\zeta_{n} \notin U_{S}\left(k_{n}\right) \cdot \mu\left(k_{n}(\sqrt{-1})\right) .
$$

Это означает, что $\varepsilon_{\infty}(1) \notin U_{S}\left(k_{\infty}\right)$. Поскольку из определения группы $\widetilde{C}\left(k_{\infty}\right)$ следует, что $\varepsilon_{\infty}(1) \in \widetilde{C}_{S}\left(k_{\infty}\right)$, то мы получаем, что первые два индекса в $(4.2)$ равны 2. Пусть $n_{0}$ - наибольший индекс такой, что $\zeta_{n_{0}} \in k(\sqrt{-1})$. Следовательно, естественная проекция $\widetilde{C}_{S}\left(k_{\infty}\right) \rightarrow \widetilde{C}_{S}(k)$ переводит $\varepsilon_{\infty}(1)$ в $\left(1-\zeta_{n_{0}}\right)$. Тогда из (4.6) следует, что $\widetilde{U}_{S}^{*}(k) \neq U_{S}^{*}(k), \widetilde{C}_{S}(k) \neq C_{S}(k)$. Так как $\left[\widetilde{U}_{S}\left(k_{\infty}\right): U_{S}\left(k_{\infty}\right)\right] \geqslant\left[\widetilde{U}_{S}^{*}(k):\right.$ 
О ФОРМУЛАХ ДЛЯ ЧИСЛА КЛАССОВ ВЕЩЕСТВЕННЫХ АБЕЛЕВЫХ ПОЛЕЙ 67

$\left.U_{S}^{*}(k)\right]$ и $\left[\widetilde{C}_{S}\left(k_{\infty}\right): C_{S}\left(k_{\infty}\right)\right] \geqslant\left[\widetilde{C}_{S}(k): C_{S}(k)\right]$, то мы получаем, что последние два индекса в (4.2) также равны 2. Этим формула (4.2) доказана полностью.

Чтобы доказать (4.3), достаточно заметить, что $\widetilde{U}_{S}(k)=U_{S}^{*}(k)\left\langle\left(1-\zeta_{n_{0}}\right)\right\rangle$ и $\widetilde{C}_{S}(k)=C_{S}(k)\left\langle\left(1-\zeta_{n_{0}}\right)\right\rangle$. Предложение доказано.

ПрЕДЛОЖЕНИЕ 4.2. Существуют естественные изоморфизмы конечных абелевых групп

$$
\begin{aligned}
\left(\widetilde{H}\left(k_{\infty}\right) / \widetilde{C}_{S}(k)\right)_{(0)} & \cong \widetilde{H}(k) / \widetilde{C}_{S}(k), \\
\left(\widetilde{U}_{S}\left(k_{\infty}\right) / \widetilde{C}_{S}\left(k_{\infty}\right)\right)_{(0)} & \cong \widetilde{U}_{S}(k) / \widetilde{C}_{S}(k) .
\end{aligned}
$$

ДокАЗАТЕЛЬство. Первая формула уже доказана (см. (4.5)). Вторая может быть доказана тем же способом, используя тот факт, что группа $\widetilde{U}_{S}\left(k_{\infty}\right)$ является $\Lambda$-свободным и, следовательно, $\bar{\Gamma}$-свободным модулем.

ПРЕДЛОЖЕНИЕ 4.3. (см. [9, предложение 7.5]). Для любого поля алгебраических чисел $k$ существует естественный изоморфизм

$$
T_{\ell}\left(k_{\infty}\right)^{\bar{\Gamma}} \cong \widetilde{U}_{S}(k) / U_{S}^{*}(k) .
$$

Для удобства читателя мы дадим здесь короткое замкнутое доказательство этого утверждения.

ДокАЗАТЕЛЬСТво. Пусть $\widetilde{X}$ - группа Галуа максимального абелева $\ell$-расширения поля $k_{\infty}$ и $\widetilde{W}$ - подгруппа группы $\widetilde{X}$, порожденная подгруппами инерции для точек $v \nmid \ell$ и подгрупами разложения для точек $v \mid \ell$. Тогда имеется точная последовательность

$$
0 \longrightarrow \widetilde{W} \longrightarrow \widetilde{X} \longrightarrow T_{\ell}\left(k_{\infty}\right) \longrightarrow 0 .
$$

Как хорошо известно, $\widetilde{X}^{\bar{\Gamma}}=0$, поэтому (4.7) индуцирует точную последовательность

$$
0 \longrightarrow T_{\ell}\left(k_{\infty}\right)^{\bar{\Gamma}} \longrightarrow \widetilde{W}_{(0)} \stackrel{\beta}{\longrightarrow} \widetilde{X}_{(0)} \stackrel{\alpha}{\longrightarrow} T_{\ell}\left(k_{\infty}\right)_{(0)} \longrightarrow 0 .
$$

Положим

$$
\widetilde{H}\left(k_{v}\right)=\bigcap_{n=1}^{\infty} N_{k_{n, v} / k_{v}}\left(k_{n, v}^{*}[\ell]\right), \quad E(k)=\left(\prod_{v \nmid \ell} U\left(k_{v}\right)[\ell]\right) \times\left(\prod_{v \mid \ell} \widetilde{H}\left(k_{v}\right)\right) .
$$

Отметим, что для вещественной точки $v$ при $\ell=2$ мы имеем $U\left(k_{v}\right)[\ell]=\{ \pm 1\}$, а для комплексной точки $v$ имеем $U\left(k_{v}\right)[\ell]=0$ при любом $\ell$.

Положим $\widehat{\mathcal{U}}_{S}(k)=U_{S}(k)[\ell] \cap E(k)$. Тогда точна последовательность

$$
1 \longrightarrow \mu_{\ell}(k) \longrightarrow \widehat{\mathcal{U}}_{S}(k) \longrightarrow \widehat{U}_{S}(k) \longrightarrow 1 .
$$

Мы определим группы $E\left(k_{n}\right)$ и $\widehat{\mathcal{U}}_{S}\left(k_{n}\right)$ аналогичным образом. Применяя теорию полей классов, мы получаем, что

$$
\operatorname{Im} \beta=\operatorname{Ker} \alpha \cong E(k) / \widehat{\mathcal{U}}_{S}(k) .
$$


По этой же причине мы получаем

$$
\widetilde{W}=\lim _{\longleftarrow}\left(E\left(k_{n}\right) / \widehat{\mathcal{U}}_{S}\left(k_{n}\right)\right)=\left(\lim _{\longleftarrow} E\left(k_{n}\right)\right) /\left(\lim _{\longleftarrow} U_{S}\left(k_{n}\right)[\ell]\right) .
$$

Поскольку $E\left(k_{n}\right)$ является когомологически тривиальным $G\left(k_{n} / k\right)$-модулем, мы получаем из (4.9)

$$
\widetilde{W}_{(0)} \cong E(k) / \mathcal{U}_{S}^{*}(k),
$$

где $\mathcal{U}_{S}^{*}(k)$ - образ естественной проекции $\lim _{U_{S}}\left(k_{n}\right)[\ell] \rightarrow \widehat{\mathcal{U}}_{S}(k)$. Отметим, что $\beta$ индуцировано тождественным отображением id: $E(k) \rightarrow E(k)$. Из (4.8) и (4.9) следует

$$
T_{\ell}\left(k_{\infty}\right)^{\bar{\Gamma}} \cong \operatorname{Ker} \beta \cong \widehat{\mathcal{U}}_{S}(k) / \mathcal{U}_{S}^{*}(k)=\widetilde{U}_{S}(k) / U_{S}^{*}(k) .
$$

Предложение доказано.

Для вещественного абелева поля $k$ с группой Галуа $G=G(k / \mathbf{Q})$ положим $G=G_{\ell} \times G_{0}$, где $G_{\ell}-$ силовская $\ell$-подгруппа группы $G$ и $\left(\left|G_{0}\right|, \ell\right)=1$. Пусть $\Phi-$ множество всех $\mathbf{Q}_{\ell}$-неприводимых характеров группы $G_{0}$.

ТЕОРема 4.1 (теорема 3 введения). Для любого вещественного абелева поля $k$ справедливо равенство

$$
\left|T_{\ell}\left(k_{\infty}\right)_{(0)}\right|=\left[\widehat{U}_{S}(k): C_{S}(k)\right] .
$$

Для любого $\varphi \in \Phi$ справедливо равенство

$$
\left|T_{\ell}\left(k_{\infty}\right)_{(0), \varphi}\right|=\left[\widehat{U}_{S}(k)_{\varphi}: C_{S}(k)_{\varphi}\right] .
$$

ДОКАЗАТЕЛЬСТВО. Пусть $P$ - максимальный конечньй подмодуль модуля $T_{\ell}\left(k_{\infty}\right)$ и $R:=T_{\ell}\left(k_{\infty}\right) / P$. Тогда для любого $\varphi \in \Phi$ сушествует точная последовательность

$$
0 \longrightarrow P_{\varphi} \longrightarrow T_{\ell}\left(k_{\infty}\right)_{\varphi} \longrightarrow R_{\varphi} \longrightarrow 0
$$

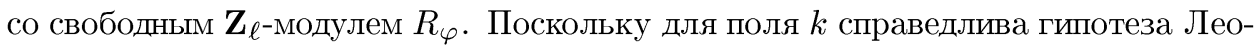
польдта, мы имеем $R^{\bar{\Gamma}}=0$. Следовательно, (4.11) индуцирует точную последовательность конечных абелевых груп

$$
0 \longrightarrow P_{(0), \varphi} \longrightarrow T_{\ell}\left(k_{\infty}\right)_{(0), \varphi} \longrightarrow R_{(0), \varphi} \longrightarrow 0 .
$$

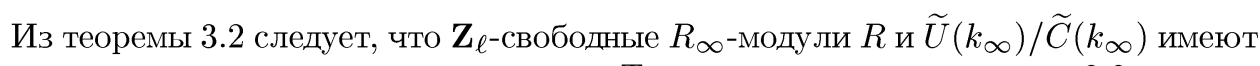
одинаковые характеристические ряды. Тогда согласно предложению 2.3 для любого характера $\varphi \in \Phi$ мы имеем

$$
\left|\left(\widetilde{U}\left(k_{\infty}\right) / \widetilde{C}\left(k_{\infty}\right)\right)_{(0), \varphi}\right|=\left|R_{(0), \varphi}\right| .
$$

Из предложений 4.1 и 4.2 следует, что

$$
\left(\widetilde{U}\left(k_{\infty}\right) / \widetilde{C}\left(k_{\infty}\right)\right)_{(0), \varphi} \cong\left(U_{S}^{*}(k)_{\varphi} / C_{S}(k)_{\varphi}\right) .
$$

Таким образом,

$$
\left|R_{(0), \varphi}\right|=\left[U_{S}^{*}(k)_{\varphi}: C_{S}(k)_{\varphi}\right] .
$$

Поскольку $T_{\ell}\left(k_{\infty}\right)^{\bar{\Gamma}}=P^{\bar{\Gamma}}$, мы получаем из предложения 4.3

$$
\left|P_{(0), \varphi}\right|=\left[\widehat{U}_{S}(k)_{\varphi}: U_{S}^{*}(k)_{\varphi}\right] .
$$

Сопоставляя (4.12), (4.13) и (4.14), мы получаем последнее утверждение теоремы. Первое утверждение может быть доказано точно так же. 


\section{§5. Доказательство теоремы 4}

Пусть $k$ - некоторое вешественное абелево поле и $G=G(k / \mathbf{Q})$. Как и в $\S 4$, мы полагаем $G=G_{\ell} \times G_{0}$, где $G_{\ell}$ - силовская $\ell$-подгруппа групшы $G$ и $\left(\left|G_{0}\right|, \ell\right)=1$. Если $\chi$ - произвольный $\overline{\mathbf{Q}}_{\ell}^{*}$-значный одномерный характер группы $G$ и $\Phi$ - множество всех $\mathbf{Q}_{\ell}$-неприводимых характеров группы $G_{0}$, то запись $\chi \mid \varphi$ для $\varphi \in \Phi$ означает, что $\left.\chi\right|_{G_{0}}$ является неприводимой компонентой характера $\varphi$. Через $\varphi_{0}$ мы обозначаем тривиальньй характер группы $G_{0}$, т.е. $\varphi_{0}(\sigma)=1$ для любого $\sigma \in G_{0}$.

Положим

$$
Y(k)=\prod_{v \mid \ell} \mu_{\ell}\left(k_{v}\right)
$$

ТЕОРема 5.1 (теорема 4 введения). Для любого вещественного абелева поля $k$ и любого $\varphi \in \Phi$ справедливы равенства

$$
\begin{gathered}
\nu_{\ell}\left(\left|T_{\ell}\left(k_{\infty}\right)_{(0)}\right|\right)=\nu_{\ell}\left(\left[H(k): \widehat{U}_{S}(k)\right]^{-1}|Y(k)|^{-1} \ell^{t} \prod_{\substack{\chi \in \widehat{G} \\
\chi \neq 1}} \frac{1}{2} L_{\ell}(1, \chi)\right), \\
\nu_{\ell}\left(\left|T_{\ell}\left(k_{\infty}\right)_{(0), \varphi}\right|\right)=\nu_{\ell}\left(\left[H(k)_{\varphi}: \widehat{U}_{S}(k)_{\varphi}\right]^{-1}\left|Y(k)_{\varphi}\right|^{-1} \ell^{t_{\varphi}} \prod_{\substack{\chi \in \widehat{G} \\
\chi \mid \varphi, \chi \neq 1}} \frac{1}{2} L_{\ell}(1, \chi)\right),
\end{gathered}
$$

где показатели $t$ и $t_{\varphi}$ определяются равенствами

$$
t=\left\{\begin{array}{lll}
r & n p u & \ell \neq 2, \\
r+1 & n p u & \ell=2,
\end{array} \quad t_{\varphi}=\left\{\begin{array}{lll}
t & n p u & \varphi=\varphi_{0} \\
0 & n p u & \varphi \neq \varphi_{0}
\end{array}\right.\right.
$$

a r определяется из формулы (2.5).

ДоКАЗАТЕЛЬСТво. Согласно предложению 3.2 мы имеем квазиизоморфизм $\mathcal{A}\left(k_{\infty}\right) / C\left(k_{\infty}\right) \sim \widetilde{H}\left(k_{\infty}\right) / \widetilde{C}_{S}\left(k_{\infty}\right)$. Последний модуль свободен над $\mathbf{Z}_{\ell}$. Поэтому мы можем вычислять порядок

$$
\ell^{s_{\varphi}}:=\left|\left(\widetilde{H}\left(k_{\infty}\right) / \widetilde{C}_{S}\left(k_{\infty}\right)\right)_{(0), \varphi}\right|
$$

используя формулу $(2.7)$, где $A_{(0)}:=A /(\bar{\gamma}-1) A$ для любого $\bar{\Gamma}$-модуля $A$. Так как $\mathcal{A}\left(k_{\infty}\right) / C\left(k_{\infty}\right) \sim\left(\mathcal{A}_{\infty}^{+} / C_{\infty}\right)^{H}$, мы получаем

$$
s_{\varphi}=\nu_{\ell}\left(\prod_{\substack{\theta \in \widehat{V / H} \\ \theta \mid \varphi}} \prod_{\zeta^{\ell^{r}}=1} h\left(\zeta \zeta_{\theta}^{-1}-1, \theta\right)\right),
$$

где $h(T, \theta)$ - характеристические ряды модуля $\mathcal{A}_{\infty}^{+} / C_{\infty}$, вычисленные в теореме $2.1, H=G\left(K_{\infty} / k_{\infty}\right), V=G\left(K_{0} / \mathbf{Q}\right), \zeta_{\theta}$ определяется из равенства $\zeta_{\theta}^{\ell^{r}}=\theta(\sigma)$ и $r, \sigma$ имеют тот же смысл, что и в $(2.5)$.

Сопоставляя формулы $(2.11),(2.12)$ и (5.4), мы получаем, что

$$
s_{\varphi}=\left\{\begin{array}{lll}
\nu_{\ell}\left(a_{\varphi} b_{\varphi}^{-1}\right) & \text { при } \varphi \neq \varphi_{0}, \\
\nu_{\ell}\left(c_{\varphi_{0}} b_{\varphi_{0}}^{-1}\right) & \text { при } \varphi=\varphi_{0},
\end{array}\right.
$$


где

$$
\begin{gathered}
a_{\varphi}:=\prod_{\substack{\theta \in \overline{V / H} \\
\theta \mid \varphi}} \prod_{\zeta^{\ell^{r}}=1} f\left(\kappa \zeta_{\theta} \zeta^{-1}-1, \bar{\theta} \omega\right), \\
b_{\varphi}=\prod_{\substack{\theta \in \widehat{V / H} \\
\theta \mid \varphi}} \prod_{\zeta^{\ell r}=1} y\left(\zeta \zeta_{\theta}^{-1}-1, \theta\right), \\
c_{\varphi_{0}}=\left[\prod_{\substack{\theta \in \overline{V / H} \\
\theta \mid \varphi_{0}, \theta \neq 1}} \prod_{\zeta^{\ell^{r}}=1} f\left(\kappa \zeta_{\theta} \zeta^{-1}-1, \bar{\theta} \omega\right)\right] \times \prod_{\zeta^{\ell r}=1} \eta(\zeta-1, \omega)
\end{gathered}
$$

и $b_{\varphi_{0}}$ определяется формулой (5.7) при $\varphi=\varphi_{0}$.

Пусть $\chi$ - произвольный характер группы $G_{\infty}$, который четен как характер Дирихле. Тогда характер $\chi$ можно представить в виде $\chi=\theta \pi$, где $\theta$ и $\pi$ - характеры первого и второго рода в смысле Ивасавы соответственно. Согласно данному в [5] знаменитому соотношению Ивасавы между $\ell$-адическими $L$-функциями и рядами Ивасавы мы имеем

$$
\frac{1}{2} L_{\ell}(s, \chi)=f\left(\xi \kappa^{s}-1, \bar{\theta} \omega\right),
$$

где $L_{\ell}(s, \chi)-\ell$-адическая $L$-функция Куботы-Леопольдта, соответствуюшая характеру $\chi$, и $\xi=\chi(\gamma)^{-1}=\pi(\gamma)^{-1}$.

Заметим, что $\chi$ является характером групшы $G=G(k / \mathbf{Q})$ тогда и только тогда, когда $\left.\theta\right|_{H}=1$, т.е. $\theta$ является характером группы $V / H$, и $\chi(\bar{\gamma})=1$, т.е. $\chi\left(\gamma^{\ell^{r}} \sigma\right)=$ $\pi(\gamma)^{\ell^{r}} \theta(\sigma)=1$. Таким образом, все характеры $\chi$ группы $G$ с заданным $\theta$ имеют вид $\theta \pi$, где $\pi(\gamma)^{-1}=\zeta_{\theta} \zeta^{-1}$ и $\zeta$ пробегает все корни из единицы степени $\ell^{r}$.

Тогда из (5.6) и (5.9) следует

$$
a_{\varphi}=\prod_{\substack{\chi \in \widehat{G} \\ \chi \mid \varphi}} \frac{1}{2} L_{\ell}(1, \chi)
$$

Чтобы вычислить $c_{\varphi_{0}}$, заметим, что, рассуждая, как и прежде, мы получаем

$$
\prod_{\substack{\theta \in \widehat{V / H} \\ \theta \mid \varphi_{0}, \theta \neq 1}} \prod_{\zeta^{\ell^{r}}=1} f\left(\kappa \zeta_{\theta} \zeta^{-1}-1, \bar{\theta} \omega\right)=\prod_{\substack{\chi \in \widehat{G}, \chi=\theta \pi \\ \theta \mid \varphi_{0}, \theta \neq 1}} \frac{1}{2} L_{\ell}(1, \chi),
$$

где $\chi$ пробегает все характеры группы $G$ вида $\chi=\theta \pi \mathrm{c} \theta \mid \varphi_{0}$ и $\theta \neq 1$.

Чтобы вычислить последнее произведение в (5.8), заметим, что согласно (2.12) мы имеем

$$
\begin{aligned}
\prod_{\zeta^{\ell r}=1} \eta(\kappa \zeta-1, \omega) & =\eta(\kappa-1, \omega) \times \prod_{\substack{\zeta^{r}=1 \\
\zeta \neq 1}}[(1-\zeta) \cdot f(\kappa \zeta-1, \omega)] \\
& =\ell^{r} \eta(\kappa-1, \omega) \times \prod_{\substack{\chi \in \widehat{G} \\
\chi=\theta \pi, \theta=1}} \frac{1}{2} L_{\ell}(1, \chi) .
\end{aligned}
$$


Таким образом, в последнем произведении $\chi$ пробегает все элементы $\widehat{G}$, являюшиеся характерами второго рода.

Сопоставляя (5.11) и (5.12) и учитывая, что ряд $\eta(T, \omega)$ обратим, мы получаем

$$
c_{\varphi_{0}}=\varepsilon \ell^{r} \prod_{\substack{\chi \in \widehat{G} \\ \chi \mid \varphi_{0}, \chi \neq 1}} \frac{1}{2} L_{\ell}(1, \chi)
$$

с некоторым обратимым элементом $\varepsilon$.

Чтобы вычислить $\nu_{\ell}\left(b_{\varphi}\right)$, заметим, что согласно (2.7) мы имеем

$$
\nu_{\ell}\left(b_{\varphi}\right)=\nu_{\ell}\left(\left|\left(Y_{\infty}^{H}\right)_{(0), \varphi}\right|\right)
$$

Положим

$$
Y\left(k_{n}\right):=\prod_{v \mid \ell} \mu_{\ell}\left(k_{n, v}\right) \quad \text { и } \quad Y\left(k_{\infty}\right):=\lim _{\longleftarrow} Y\left(k_{n}\right),
$$

где проективный предел берется относительно норменных отображений. Тогда $Y_{\infty}^{H} \cong Y\left(k_{\infty}\right)$, поэтому $\left(Y_{\infty}^{H}\right)_{(0)} \cong Y\left(k_{\infty}\right)_{(0)}$. Если $\ell \neq 2$ и $\zeta_{0} \notin k_{v}$, то $\zeta_{0} \notin k_{n, v}$ при любом $n$, и мы имеем $Y\left(k_{\infty}\right)_{(0)}=Y(k)=0$. Если $\ell \neq 2$ и $\zeta_{0} \in k_{v}$, то $Y\left(k_{n}\right)$ является когомологически тривиальным $G\left(k_{n} / k\right)$-модулем, следовательно, $Y\left(k_{\infty}\right)_{(0)} \cong Y(k)$.

Если $\ell=2$ и $\sqrt{-1} \notin k_{\infty, v}$, то $Y\left(k_{\infty}\right)=0$. Если $\ell=2$ и $\sqrt{-1} \in k_{v}$, то групшы $Y\left(k_{n}\right)$ снова будут когомологически тривиальными, и мы получаем, что $Y\left(k_{\infty}\right)_{(0)} \cong Y(k)$.

Наконец, если $\ell=2, \sqrt{-1} \notin k_{v}$ и $\sqrt{-1} \in k_{\infty, v}$, то мы получаем снова $Y\left(k_{\infty}\right)_{(0)} \cong Y(k)$. В самом деле, если любая точка $v \mid \ell$ поля $k$ не распадается в $k_{\infty} / k$, то $\bar{\gamma}$ действует на групп $Y\left(k_{\infty}\right)$, как умножение на некоторое число $\lambda \in \mathbf{Z}_{2}^{*}$. Без труда проверяется, что $\lambda \not \equiv 1(\bmod 4)$, поэтому $Y\left(k_{\infty}\right)_{(0)} \cong$ $Y\left(k_{\infty}\right) / 2 Y\left(k_{\infty}\right) \cong Y(k)$. Обший случай может быть без труда сведен к уже рассмотренному. Таким образом, мы получаем, что с точностью до умножения на некоторую единицу

$$
b_{\varphi}= \begin{cases}1 & \text { при } \ell=2 \text { и } \sqrt{-1} \notin k_{\infty, v} \text { для } v \mid \ell, \\ \left|Y(k)_{\varphi}\right| & \text { в противном случае. }\end{cases}
$$

Заметим, что при $\ell \neq 2$ мы имеем $b_{\varphi_{0}}=1$, поскольку в этом случае $Y(k)_{\varphi_{0}}=1$. Поэтому из $(5.3),(5.5),(5.10),(5.13)$ и (5.14) следует, что

$$
\left[\widetilde{H}(k)_{\varphi}: \widetilde{C}(k)_{\varphi}\right]=\left|\left(\widetilde{H}\left(k_{\infty}\right) / \widetilde{C}\left(k_{\infty}\right)\right)_{(0), \varphi}\right|=\ell^{s_{\varphi}}
$$

где при $\ell \neq 2 s_{\varphi}$ задается формулой

$$
s_{\varphi}= \begin{cases}\nu_{\ell}\left(\left(\prod_{\substack{\chi \in \widehat{G} \\ \chi \mid \varphi}} \frac{1}{2} L_{\ell}(1, \chi)\right)\left|Y(k)_{\varphi}\right|^{-1}\right) & \text { при } \varphi \neq \varphi_{0}, \\ \nu_{\ell}\left(\ell^{r} \prod_{\substack{\chi \in \widehat{G} \\ \chi \varphi_{0}, \chi \neq 1}} \frac{1}{2} L_{\ell}(1, \chi)\right) & \text { при } \varphi=\varphi_{0} .\end{cases}
$$


Если $\ell=2$, то $s_{\varphi}$ задается формулой

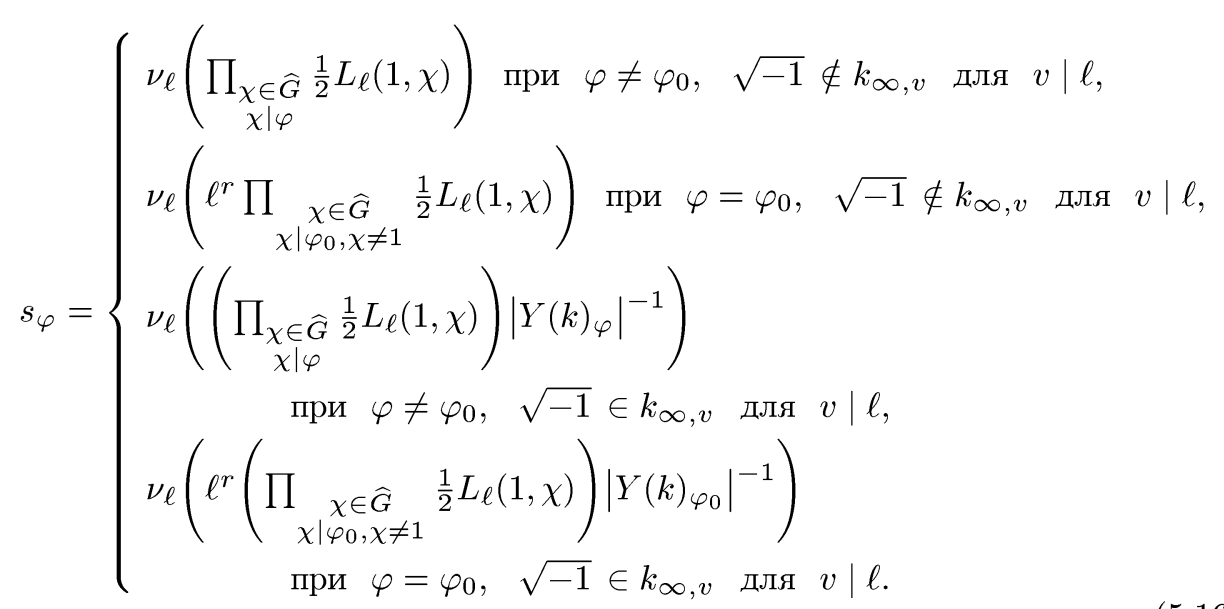

Согласно теореме 4.1 мы получаем

$$
\begin{aligned}
\left|T_{\ell}\left(k_{\infty}\right)_{(0)}\right| & =\left[H(k): C_{S}(k)\right]\left[H(k): \widehat{U}_{S}(k)\right]^{-1} \\
\left|T_{\ell}\left(k_{\infty}\right)_{(0), \varphi}\right| & =\left[H(k)_{\varphi}: C_{S}(k)_{\varphi}\right]\left[H(k)_{\varphi}: \widehat{U}_{S}(k)_{\varphi}\right]^{-1}
\end{aligned}
$$

для любого $\varphi$.

Если $\ell \neq 2$, то $\widetilde{H}\left(k_{\infty}\right)=H\left(k_{\infty}\right)$ и $\widetilde{C}\left(k_{\infty}\right)=C\left(k_{\infty}\right)$. Поэтому в этом случае $\left(\widetilde{H}\left(k_{\infty}\right) / \widetilde{C}\left(k_{\infty}\right)\right)_{(0)}=\left(H\left(k_{\infty}\right) / C\left(k_{\infty}\right)\right)_{(0)} \cong H(k) / C(k)$. Таким образом, при $\ell \neq 2$ утверждение теоремы следует из (5.3) и (5.15).

Если $\ell=2$ и $\sqrt{-1} \notin k_{\infty, v}$ для $v \mid \ell$, то $\widetilde{H}(k) / H(k) \cong{ }_{2} Y(k)=Y(k)$, где ${ }_{2} Y(k)=\{y \in Y(k) \mid 2 y=0\}$ и $\widetilde{C}_{S}(k) / C_{S}(k) \cong \mathbf{Z} / 2 \mathbf{Z}$. Если $\sqrt{-1} \in k_{\infty, v}$ для $v \mid \ell$, то $\widetilde{H}(k)=H(k)$ и $\widetilde{C}_{S}(k) / C_{S}(k) \cong \mathbf{Z} / 2 \mathbf{Z}$. Следовательно,

$$
\left[H(k): C_{S}(k)\right]= \begin{cases}2|Y(k)|^{-1}\left[\widetilde{H}(k): \widetilde{C}_{S}(k)\right] & \text { при } \sqrt{-1} \notin k_{\infty, v} \text { для } v \mid \ell, \\ 2\left[\widetilde{H}(k): \widetilde{C}_{S}(k)\right] & \text { при } \sqrt{-1} \in k_{\infty}, v \text { для } v \mid \ell .\end{cases}
$$

Если $\varphi \neq \varphi_{0}$, то

$$
\left[H(k)_{\varphi}: C_{S}(k)_{\varphi}\right]=\left\{\begin{array}{c}
\left|Y(k)_{\varphi}\right|^{-1}\left[\widetilde{H}(k)_{\varphi}: \widetilde{C}_{S}(k)_{\varphi}\right] \\
\text { при } \sqrt{-1} \notin k_{\infty, v} \text { для } v \mid \ell, \\
{\left[\widetilde{H}(k)_{\varphi}: \widetilde{C}_{S}(k)_{\varphi}\right]} \\
\text { при } \sqrt{-1} \in k_{\infty, v} \text { для } v \mid \ell .
\end{array}\right.
$$

Если $\varphi=\varphi_{0}$, то

$$
\left[H(k)_{\varphi_{0}}: C_{S}(k)_{\varphi_{0}}\right]=\left\{\begin{array}{c}
2\left|Y(k)_{\varphi_{0}}\right|^{-1}\left[\widetilde{H}(k)_{\varphi_{0}}: \widetilde{C}_{S}(k)_{\varphi_{0}}\right] \\
\text { при } \sqrt{-1} \notin k_{\infty, v} \text { для } v \mid \ell, \\
2\left[\widetilde{H}(k)_{\varphi_{0}}: \widetilde{C}_{S}(k)_{\varphi_{0}}\right] \\
\text { при } \sqrt{-1} \in k_{\infty, v} \text { для } v \mid \ell .
\end{array}\right.
$$

Теперь утверждение нашей теоремы для $\ell=2$ следует из (5.3) и (5.16). 


\section{§6. Уточнение теоремы Хассе о дискриминанте и ведущем модуле}

Пусть $k$ - поле алгебраических чисел степени $t$, являющееся расширением Галуа над $\mathbf{Q}$ и имеющее группу Галуа $G=G(k / \mathbf{Q})$. Мы будем рассматривать $k$ как подполе некоторого фиксированного алгебраического замыкания $\overline{\mathbf{Q}}_{\ell}$ поля $\mathbf{Q}_{\ell}$. Положим

$$
\mathbf{A}_{k}=\left\{\left(x_{\sigma_{1}}, \ldots, x_{\sigma_{t}}\right) \mid x_{\sigma_{i}} \in \overline{\mathbf{Q}}_{\ell}, \quad G=\left\{\sigma_{1}=1, \sigma_{2}, \ldots, \sigma_{t}\right\}\right\} .
$$

Таким образом, $\mathbf{A}_{k}$ совпадает с множеством всех векторов длины $t$ с координатами из $\overline{\mathbf{Q}}_{\ell}$, имеющих $G$ в качестве множества индексов. Мы определим сложение и умножение в $\mathbf{A}_{k}$ покомпонентно. Для $\lambda \in \overline{\mathbf{Q}}_{\ell}$ и $\sigma \in G$ мы полагаем $\lambda\left(x_{\sigma_{1}}, \ldots, x_{\sigma_{t}}\right)=\left(\lambda x_{\sigma_{1}}, \ldots, \lambda x_{\sigma_{t}}\right)$,

$$
\sigma\left(x_{\sigma_{1}}, \ldots, x_{\sigma_{t}}\right)=\left(y_{\sigma_{1}}, \ldots, y_{\sigma_{t}}\right), \quad \text { где } y_{\sigma_{i}}=x_{\sigma_{i} \sigma} \text { для } i=1, \ldots, t .
$$

Таким образом, действие $G$ коммутирует с умножением на $\lambda \in \mathbf{Q}_{\ell}$, и мы можем рассматривать $\mathbf{A}_{k}$ как $\overline{\mathbf{Q}}_{\ell}[G]$-алгебру.

Положим $f_{i}=\{0, \ldots, 0,1,0 \ldots, 0\} \in \mathbf{A}_{k}$, где единственная ненулевая координата имеет индекс $\sigma_{i}$. Тогда множество $\left\{f_{1}, \ldots, f_{t}\right\}$ образует базис алгебры $\mathbf{A}_{k}$ над $\overline{\mathbf{Q}}_{\ell}$ и любой из элементов $f_{i}$ порождает $\mathbf{A}_{k}$ как $\overline{\mathbf{Q}}_{\ell}[G]$-модуль. Это означает, что $\mathbf{A}_{k} \cong \overline{\mathbf{Q}}_{\ell}[G]$ как $\overline{\mathbf{Q}}_{\ell}[G]$-модуль, причем мы имеем $\sigma\left(f_{i}\right)=f_{j}$, где $\sigma_{j}=\sigma_{i} \sigma$.

Мы определим вложение $\alpha: k \hookrightarrow \mathbf{A}_{k}$ при помоши формулы $\alpha(x)=\left(x_{\sigma_{1}}, \ldots\right.$ $\left.\ldots, x_{\sigma_{t}}\right)$, где $x_{\sigma_{i}}=\sigma_{i}^{-1}(x), i=1, \ldots, t$. Таким образом, мы имеем $\alpha(\sigma(x))=$ $\sigma(\alpha(x))$ для любых $\alpha \in k$ и $\sigma \in G$.

Если элементы $e_{1}, \ldots, e_{t} \in k$ образуют базис $k$ над $\mathbf{Q}$, то элементы $\alpha\left(e_{1}\right), \ldots, \alpha\left(e_{t}\right)$ образуют базис $\mathbf{A}_{k}$ над $\overline{\mathbf{Q}}_{\ell}$. Следовательно, $\alpha$ индуцирует изоморфизм

$$
\overline{\mathbf{Q}}_{\ell} \otimes_{\mathbf{Q}} k \cong \mathbf{A}_{k}, \quad \lambda \otimes x \rightarrow \lambda \alpha(x) \quad \text { для любых } \lambda \in \overline{\mathbf{Q}}_{\ell}, \quad x \in k .
$$

Если $\overline{\mathcal{O}}$ - кольцо цельх поля $\overline{\mathbf{Q}}_{\ell}$, то множество

$$
\mathbf{R}_{k}=\left\{\left(x_{\sigma_{1}}, \ldots, x_{\sigma_{t}}\right) \in \mathbf{A}_{k} \mid x_{\sigma_{i}} \in \overline{\mathcal{O}} \text { для любых } \sigma_{i} \in G\right\}
$$

совпадает с максимальным порядком в $\mathbf{A}_{k}$. Отметим, что $\mathbf{R}_{k}$ является свободным $\overline{\mathcal{O}}[G]$-модулем ранга 1 , порождаемым любым из элементов $f_{1}, \ldots, f_{t}$.

Если $\mathcal{O}_{k}$ - кольцо целых поля $k$ и элементы $\left\{e_{1}, \ldots, e_{t}\right\}$ образуют $\mathbf{Z}$-базис $\mathcal{O}_{k}$, то $\left\{\alpha\left(e_{1}\right), \ldots, \alpha\left(e_{n}\right)\right\}$ образуют $\overline{\mathcal{O}}$-базис модуля $\mathcal{O}_{k}:=\overline{\mathcal{O}} \otimes_{\mathbf{z}} \mathcal{O}_{k}$.

Пусть $V$-некоторое $\overline{\mathbf{Q}}_{\ell}$-подпространство в $\mathbf{A}_{k}$ и $L, M \subset V$-полные $\overline{\mathcal{O}}$-решетки в $V$. Это означает, что $L$ и $M$ конечно порождены как $\overline{\mathcal{O}}$-модули и каждый из них порождает $V$ как $\overline{\mathbf{Q}}_{\ell}$-пространство. Тогда сушествует невырожденное линейное преобразование $A: V \rightarrow V$ такое, что $A(L)=M$. В этом случае, как и в [15], мы определим индекс

$$
(L: M)=\operatorname{det}(A)
$$

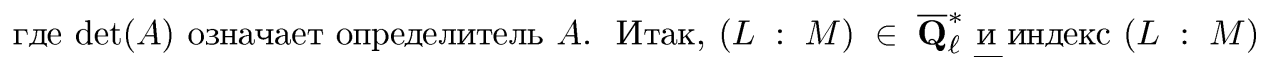
определен однозначно с точностью до умножения на единицу из $\overline{\mathcal{O}}$. Следовательно, $\nu_{\ell}((L: M))$ не зависит от выбора $A$. Если $L \subseteq M$, то $\nu_{\ell}((L: M))=0$ тогда и только тогда, когда $L=M$.

Мы определим $\overline{\mathbf{Q}}_{\ell}$-билинейную форму $\psi: \mathbf{A}_{k} \times \mathbf{A}_{k} \rightarrow \overline{\mathbf{Q}}_{\ell}$ при помощи формулы $\psi(x, y)=\sum_{\sigma_{i} \in G} x_{\sigma_{i}} y_{\sigma_{i}}$ для $x=\left(x_{\sigma_{1}}, \ldots, x_{\sigma_{t}}\right), y=\left(y_{\sigma_{1}}, \ldots, y_{\sigma_{t}}\right) \in \mathbf{A}_{k}$. 
Тогда для любых $a, b \in k$ мы имеем

$$
\psi(\alpha(a), \alpha(b))=\operatorname{Sp}_{k / \mathbf{Q}}(a b),
$$

где $\mathrm{Sp}_{k / \mathbf{Q}}$ означает след из $k$ в $\mathbf{Q}$.

Пусть $A$ - матрица $\overline{\mathbf{Q}}_{\ell}$-линейного преобразования такого, что $A f_{i}=\alpha\left(e_{i}\right), i=$ $1, \ldots, t$, где $\left\{f_{1}, \ldots, f_{t}\right\}$ и $\left\{\alpha\left(e_{1}\right), \ldots, \alpha\left(e_{t}\right)\right\}$ определены, как и вьше.

Форма $\psi$ имеет матрищу $E=1$ относительно базиса $\left\{f_{1}, \ldots, f_{t}\right\}$ и матрицу $C=\left(\operatorname{Sp}_{k / \mathbf{Q}}\left(e_{i} e_{j}\right)\right)$ относительно базиса $\left\{e_{1}, \ldots, e_{t}\right\}$. Поэтому $(\operatorname{det}(A))^{2}=\operatorname{det}(C)$ и, следовательно, $\left(\mathbf{R}_{k}: \widetilde{\mathcal{O}}_{k}\right)^{2}=d_{k}$, где $d_{k}$ - абсолютньй дискриминант поля $k$.

Начиная с этого места, мы предполагаем, что поле $k$ абелево. Согласно теореме Хассе о дискриминанте и ведущем модуле справедливо равенство

$$
d_{k}=\prod_{\chi \in \widehat{G}} f_{\chi}=\prod_{\substack{\chi \in \widehat{G} \\ \chi \neq 1}} f_{\chi}
$$

где $\widehat{G}$ - группа характеров групшы $G$ и $f_{\chi}$ - кондуктор характера $\chi$, рассматриваемого как примитивный характер Дирихле. Мы считаем, что все характеры $G$ принимают значения в $\overline{\mathbf{Q}}_{\ell}$. Для заданного $\chi \in \widehat{G}$ пусть $\zeta$ - некоторый первобразньй корень из единицы в $\overline{\mathbf{Q}}_{\ell}$ степени $f_{\chi}$. Тогда можно образовать гауссову сумму

$$
g_{\chi}=\sum_{\substack{a \bmod f_{\chi} \\\left(a, f_{\chi}\right)=1}} \chi(a) \zeta^{a} \in \overline{\mathbf{Q}}_{\ell} .
$$

Хорошо известно, что $\nu_{\ell}\left(g_{\chi}\right)$ не зависит от конкретного выбора корня $\zeta$. Хорошо известно также, что $g_{\chi} \bar{g}_{\chi}=f_{\chi}$ и $\bar{g}_{\chi}=\chi(-1) g_{\bar{\chi}}$, где $\bar{\chi}=\chi^{-1}$ и

$$
\bar{g}_{\chi}=\sum_{\substack{a \bmod f_{\chi} \\\left(a, f_{\chi}\right)=1}} \bar{\chi}(a) \zeta^{-a} .
$$

Тогда “ $\ell$-часть” теоремы Хассе о дискриминанте и ведущем модуле можно сформулировать в виде равенства

$$
\nu_{\ell}\left(\left(\mathbf{R}_{k}: \widetilde{\mathcal{O}}_{k}\right)\right)=\nu_{\ell}\left(\prod_{\substack{\chi \in \widehat{G} \\ \chi \neq 1}} g_{\chi}\right) .
$$

Пусть $\Phi$ определено, как и вьше. Следуюший результат является уточнением формулы (6.2).

ТЕОРема 6.1. Для любого $\varphi \in \Phi$ индекс $\left(\mathbf{R}_{k, \varphi}: \widetilde{\mathcal{O}}_{k, \varphi}\right)$ определен и выполняется равенство

$$
\nu_{\ell}\left(\left(\mathbf{R}_{k, \varphi}: \widetilde{\mathcal{O}}_{k, \varphi}\right)\right)=\nu_{\ell}\left(\prod_{\substack{\chi \in \widehat{G} \\ \chi \mid \varphi, \chi \neq 1}} g_{\bar{\chi}}\right) .
$$


ДокАЗАТЕЛЬСТво. Пусть поле $k$ имеет кондуктор $N=\ell^{m} d,(d, \ell)=1$. Мы докажем нашу теорему индукцией по $m$.

1. Если $N=d$ (т.е. $m=0$ ), то $d_{k}$ взаимно прост с $\ell$ и $\mathbf{R}_{k}=\widetilde{\mathcal{O}}_{k}$. Тогда $\mathbf{R}_{k, \varphi}=$ $\widetilde{\mathcal{O}}_{k, \varphi}$ и $\nu_{\ell}\left(\left(\mathbf{R}_{k, \varphi}: \widetilde{\mathcal{O}}_{k, \varphi}\right)\right)=0$ для любого $\varphi \in \Phi$. С другой стороны, соотношение $g_{\chi} g_{\bar{\chi}}=\chi(-1) f_{\chi}, f_{\chi} \mid d$, выполняющееся для целых алгебраических чисел $g_{\chi}$, $g_{\bar{\chi}}$, показывает, что $\nu_{\ell}\left(g_{\chi}\right)=0$ для любого $\chi$. Это доказьвает равенство (6.3) для $m=0$.

2. Пусть $\ell$ нечетно. Мы утверждаем, что для того, чтобы доказать (6.3) для поля $k$, достаточно доказать (6.3) для поля $k_{1}=k\left(\zeta_{0}\right)$, где $\zeta_{0}-$ первообразный корень из единищы степени $\ell$. Положим $G_{1}=G\left(k_{1} / \mathbf{Q}\right), H_{1}=G\left(k_{1} / k\right)$. Тогда $H_{1}$ - циклическая группа порядка, взаимно простого с $\ell$. Для $\varphi \in \Phi$ и $\chi \in \widehat{G}$ мы обозначим через $\varphi_{1}$ (соответственно $\chi_{1}$ ) образы элементов $\varphi$ (соответственно $\chi$ )

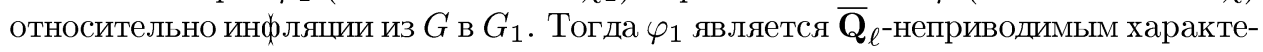
ром группы $G_{1}$, тривиальным на $H_{1}$ и на силовской $\ell$-подгруппе группы $G_{1}$. Таким образом, любой $\chi^{\prime} \in \widehat{G_{1}}$ такой, что $\chi^{\prime} \mid \varphi_{1}$, является инфлящией некоторого характера $\chi \in \widehat{G}$ такого, что $\chi \mid \varphi$. Поскольку $\chi$ и $\chi_{1}$ имеют одинаковый кондуктор, мы получаем, что $g_{\chi}=g_{\chi 1}$. Следовательно,

$$
\prod_{\substack{\chi \in \widehat{G} \\ \chi \mid \varphi, \chi \neq 1}} g_{\bar{\chi}}=\prod_{\substack{\chi_{1} \in \widehat{G}_{1} \\ \chi_{1} \mid \varphi_{1}, \chi_{1} \neq 1}} g_{\bar{\chi}_{1}}
$$

$\mathrm{C}$ другой стороны, отображение следа $\mathrm{Sp}_{k_{1} / k}$ индуцирует изоморфизмы $\mathbf{R}_{k_{1}, \varphi_{1}} \cong$ $\mathbf{R}_{k, \varphi}, \widetilde{\mathcal{O}}_{k_{1}, \varphi_{1}} \cong \widetilde{\mathcal{O}}_{k, \varphi}$, что доказывает наше утверждение.

3. Пусть теперь $\ell$ нечетно и $m=1$, т.е. $k$ имеет только ручное ветвление. Согласно п. 2 мы можем предположить, что $k=E\left(\zeta_{0}\right)$, где поле $E$ не разветвлено над $\ell$. Тогда $k=E \cdot \mathbf{Q}\left(\zeta_{0}\right)$ и $G=G(k / \mathbf{Q}) \cong F \times \Delta$, где $F=G(E / \mathbf{Q})$, $\Delta=G\left(\mathbf{Q}\left(\zeta_{0}\right) / \mathbf{Q}\right)$. Таким образом, любой $\mathbf{Q}_{\ell}$-неприводимый характер $\varphi$ группы $G$

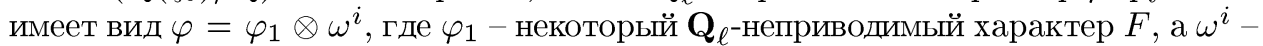
некоторая степень характера Тейхмюллера $\omega: \Delta \rightarrow \mathbf{Z}_{\ell}^{*}, i=0, \ldots, \ell-2$. Если $\chi \in \widehat{G}$ и $\chi \mid \varphi$, то $\chi=\chi_{1} \otimes \omega^{i}$, где $\chi_{1} \in \widehat{F}$ и $\chi_{1} \mid \varphi_{1}$. Разумеется, мы можем предполагать, что $i \neq 0$ (если $i=0$, то мы находимся в ситуации п. 1$)$. Поскольку $\chi_{1}$ и $\omega^{i}$ имеют взаимно простые кондукторы, выполняется равенство $g_{\bar{\chi}}=g_{\bar{\chi}_{1}} g_{\bar{\omega}^{i}}$, следовательно,

$$
\prod_{\chi \mid \varphi} g_{\bar{\chi}}=\prod_{\chi_{1} \mid \varphi_{1}} g_{\bar{\chi}_{1}} \cdot g_{\bar{\omega}^{i}}
$$

ЛЕмма 6.1. Пусть $g_{\omega^{i}} \in \overline{\mathbf{Q}}_{\ell}-$ гауссова сумма, соответствующая харакmepy $\omega^{i}$. Тогда

$$
\nu_{\ell}\left(g_{\omega^{i}}\right)=\frac{\ell-1-i}{\ell-1} \quad \text { для } \quad i=1, \ldots, \ell-2
$$

ДокАЗАТЕльСТво. Если $\omega^{i}=\bar{\omega}$, т.е. $i=\ell-2$, то, полагая $\pi_{0}=1-\zeta_{0}$, где $\zeta_{0}-$ первообразный корень из единицы степени $\ell$, мы получаем

$$
g_{\bar{\omega}}=\sum_{a=1}^{\ell-2} \bar{\omega}(a)\left(1-\pi_{0}\right)^{a} \equiv \sum_{a=1}^{\ell-2}-\bar{\omega}(a) a \pi_{0} \equiv \pi_{0} \quad\left(\bmod \pi_{0}^{2}\right) .
$$


Следовательно, $\nu_{\ell}\left(g_{\bar{\omega}}\right)=1 /(\ell-1)$.

Если автоморфизм $\delta_{b} \in \Delta$ переводит $\zeta_{0}$ в $\zeta_{0}^{b}$, то $\delta_{b}\left(g_{\omega^{i}}\right)=\bar{\omega}^{i}(b) g_{\omega^{i}}$ и $\delta_{b}\left(g_{\bar{\omega}}\right)=$ $\omega(b) g_{\bar{\omega}}$. Поэтому $\left(g_{\omega^{i}}\right)^{\ell-1} \in \mathbf{Q}_{\ell}$, и согласно теории Куммера $g_{\omega^{i}}=\left(g_{\bar{\omega}}\right)^{c} a$ для некоторых $c=1, \ldots, \ell-2$ и $a \in \mathbf{Q}_{\ell}$. Так как $\delta_{b}\left(g_{\omega^{i}}\right)=\bar{\omega}^{i}(b) g_{\omega^{i}}=\omega(b)^{c}\left(g_{\bar{\omega}}\right)^{c}$, то мы получаем, что $c=(\ell-1)-i$. Соотношение $g_{\omega^{i}} \overline{g_{\omega^{i}}}=\ell$ показывает, что $\nu_{\ell}\left(g_{\omega^{i}}\right) \leqslant 1$, поэтому $\nu_{\ell}(a)=0$. Следовательно, $\nu_{\ell}\left(g_{\omega^{i}}\right)=\frac{\ell-1-i}{\ell-1}$, что доказывает лемму.

Если $\mathcal{O}_{F}\left(\right.$ соответственно $\left.\mathcal{O}_{k}\right)$ - кольцо целых поля $F$ (соответственно поля $k$ ), то $\mathcal{O}_{k}=\mathcal{O}_{F}+\mathcal{O}_{F} \zeta_{0}+\cdots+\mathcal{O}_{F} \zeta_{0}^{\ell-2}$. Пусть

$$
e_{\omega^{i}}=|\Delta|^{-1} \sum_{\delta \in \Delta} \bar{\omega}^{i}(\delta) \delta
$$

- идемпотент, соответствующий характеру $\omega^{i}$. Тогда $e_{\omega^{i}} \zeta_{0}^{b}=|\Delta|^{-1} \omega^{i}(b) g_{\bar{\omega}^{i}}$. Следовательно, $\mathcal{O}_{k, \varphi}=g_{\bar{\omega}^{i}} \mathcal{O}_{F, \varphi_{1}}$. Учитывая, что $\widetilde{\mathcal{O}}_{k, \varphi}=\overline{\mathcal{O}} \cdot \mathcal{O}_{k, \varphi}$, мы получаем, что

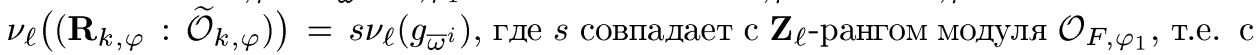
размерностью характера $\varphi_{1}$. Сравнивая это с (6.4) и с равенством леммы 6.1 и учитывая, что $\nu_{\ell}\left(g_{\bar{\chi}_{1}}\right)=0$ для любого $\chi_{1} \mid \varphi_{1}$, мы получаем справедливость $(6.3)$ в случае ручного ветвления.

4. Пусть абелево числовое поле $k$ кондуктора $N$ имеет дикое ветвление над $\ell$. Согласно п. 2 мы можем предположить, что $\zeta_{0} \in k$ для $\ell \neq 2$. В этом случае $N=\ell^{m} d$, где $m \geqslant 1$ при $\ell \neq 2$ и $m \geqslant 2$ при $\ell=2$. Положим $K_{n}=\mathbf{Q}\left(\mu_{d}, \zeta_{n}\right)$, где $n=m-1$ при $\ell \neq 2 ; n=m-2$ при $\ell=2$. Тогда $k \subseteq K_{n}$ и $k \nsubseteq K_{n-1}$, т.е., полагая $k_{0}:=k \cap K_{n-1}$, мы получаем следуюшую диаграмму полей:

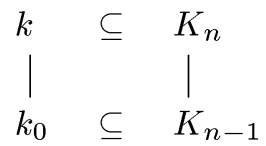

Положим $H=G\left(K_{n} / K_{n-1}\right) \cong G\left(k / k_{0}\right)$ и $F=G\left(K_{n} / k\right) \cong G\left(K_{n-1} / k_{0}\right)$. Заметим, что $H \cong \mathbf{Z} / \ell \mathbf{Z}$.

Лемма 6.2. Пусть $k$ имеет кондуктор $N \equiv 0\left(\bmod \ell^{2}\right)(N \equiv 0(\bmod 8)$ при $\ell=2)$ и $k_{0}$ имеет тот же смыслл, что и в (6.5). Пусть $\mathbf{B}_{k}-$ ядро отображения следа $\mathrm{Sp}_{k / k_{0}}: \widetilde{\mathcal{O}}_{k} \rightarrow \widetilde{\mathcal{O}}_{k_{0}}$. Тогда $\mathbf{B}_{k}$ является ииклическим $\overline{\mathcal{O}}[G]$-модулем, изоморфньцм $\mathbf{R}_{k} / \mathbf{R}_{k}^{H}, u \operatorname{Sp}_{k / k_{0}}\left(\widetilde{\mathcal{O}}_{k}\right)=\ell \widetilde{\mathcal{O}}\left(k_{0}\right) . \quad$ Eсли $K_{n} u k$ имеют тот же смиссл, что и в (6.5), то отображение $\mathrm{Sp}_{K_{n} / k}: \widetilde{\mathcal{O}}_{K_{n}} \rightarrow \widetilde{\mathcal{O}}_{k}$ индуиирует әпиморфизм $\mathbf{B}_{K_{n}} \rightarrow \mathbf{B}_{k}$.

ДокАЗАТЕльСтво. Предположим сначала, что $k=K_{n}$ (тогда $k_{0}=K_{n-1}$ ). Пусть отображение $\alpha: K_{n} \hookrightarrow \mathbf{A}_{K_{n}}$ определено, как и вьше. Тогда элементы вида $\alpha\left(\varepsilon \zeta_{n}^{s}\right), s \in \mathbf{Z}$, порождают $\widetilde{\mathcal{O}}_{K_{n}}$ как $\overline{\mathcal{O}}_{\text {-модуль. Мы имеем }}$

$$
\operatorname{Sp}_{K_{n} / K_{n-1}}\left(\alpha\left(\varepsilon \zeta_{n}^{s}\right)\right)=\left\{\begin{array}{lll}
0 & \text { при } & s \neq \equiv(\bmod \ell), \\
\ell \alpha\left(\varepsilon \zeta_{n}^{s}\right) & \text { при } s \equiv 0(\bmod \ell) .
\end{array}\right.
$$

Таким образом, элементы $\alpha\left(\varepsilon \zeta_{n}^{s}\right)$ с $s \not \equiv 0(\bmod \ell)$ порождают $\mathbf{B}_{K_{n}}$ и, следовательно,

$$
\widetilde{\mathcal{O}}_{K_{n}}=\mathbf{B}_{K_{n}} \oplus \widetilde{\mathcal{O}}_{K_{n-1}} .
$$


Поле $K_{-1}=\mathbf{Q}\left(\mu_{d}\right)$ не разветвлено над $\ell$, следовательно, $\widetilde{\mathcal{O}}_{K_{-1}}$ является свободным $\overline{\mathcal{O}}\left[G\left(K_{-1} / \mathbf{Q}\right]\right.$-модулем с одной образующей, например $x$. Тогда $x \cdot \alpha\left(\zeta_{n}\right)$ порождает $\mathbf{B}_{K_{n}}$ как $G\left(K_{n} / \mathbf{Q}\right)$-модуль, т.е. $\mathbf{B}_{K_{n}}$ - циклический модуль. Следовательно, сушествует эпиморфизм $p: \mathbf{R}_{K_{n}} \rightarrow \mathbf{B}_{K_{n}}$. Разумеется, $\mathbf{R}_{K_{n}}^{H} \subset$ Ker $p$. Поскольку модуль $\mathbf{R}_{K_{n}} / \mathbf{R}_{K_{n}}^{H}$ свободен над $\overline{\mathcal{O}}$ и имеет тот же $\overline{\mathcal{O}}$-ранг, что и $\mathbf{B}_{K_{n}}$, мы получаем, что $p$ индуцирует изоморфизм $\mathbf{B}_{K_{n}} \cong \mathbf{R}_{K_{n}} / \mathbf{R}_{K_{n}}^{H}$. Этим наша лемма доказана для случая $k=K_{n}$.

Если $k$-произвольное абелево поле, то мы имеем коммутативную диаграмму с точньми строками

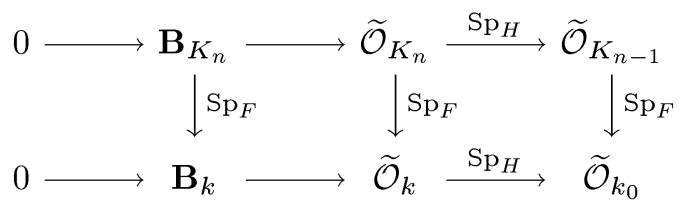

где $K_{n}, K_{n-1}$ и $k_{0}$ имеют тот же смысл, что и в (6.5).

Предположим сначала, что расширение $K_{n} / k$ не разветвлено над $\ell$. Тогда $K_{n-1} / k_{0}$ не разветвлено над $\ell$ и отображения $\mathrm{Sp}_{F}: \widetilde{\mathcal{O}}_{K_{n}} \rightarrow \widetilde{\mathcal{O}}_{k}, \operatorname{Sp}_{F}: \widetilde{\mathcal{O}}_{K_{n-1}} \rightarrow$ $\widetilde{\mathcal{O}}_{k_{0}}$ эпиморфны. Следовательно,

$$
\operatorname{Sp}_{H}\left(\widetilde{\mathcal{O}}_{k}\right)=\operatorname{Sp}_{H} \cdot \operatorname{Sp}_{F}\left(\widetilde{\mathcal{O}}_{K_{n}}\right)=\operatorname{Sp}_{F} \cdot \operatorname{Sp}_{H}\left(\widetilde{\mathcal{O}}_{K_{n}}\right)=\operatorname{Sp}_{F}\left(\ell \widetilde{\mathcal{O}}_{K_{n-1}}\right)=\ell \widetilde{\mathcal{O}}_{k_{0}} \cdot
$$

Таким образом, любой $x \in \widetilde{\mathcal{O}}_{k}$ может быть представлен в виде $x=\left(x-\ell^{-1} \mathrm{Sp}_{H} x\right)+$ $\ell^{-1} \mathrm{Sp}_{H} x$, где $\left(x-\ell^{-1} \mathrm{Sp}_{H} x\right) \in \mathbf{B}_{k}, \ell^{-1} \mathrm{Sp}_{H} x \in \widetilde{\mathcal{O}}_{k_{0}}$, следовательно, $\widetilde{\mathcal{O}}_{k}=\mathbf{B}_{k} \oplus$ $\widetilde{\mathcal{O}}_{k_{0}}$. Разложение (6.6) показывает, что отображение $\mathrm{Sp}_{F}: \mathbf{B}_{K_{n}} \rightarrow \mathbf{B}_{k}$ эпиморфно. Поэтому $\mathbf{B}_{k}$ является циклическим $\overline{\mathcal{O}}[G]$-модулем, и, рассуждая, как и прежде, мы получаем, что $\mathbf{B}_{k} \cong \mathbf{R}_{k} / \mathbf{R}_{k}^{H}$. Этим наша лемма доказана в случае, когда $K_{n} / k$ не разветвлено над $\ell$.

Заметим, что этот случай всегда имеет место, если $\ell \neq 2$ или $\ell=2$ и $n=0$. В самом деле, поскольку $\zeta_{0} \in k_{0}$ при $\ell \neq 2$ (см. п. 2), подгруппа инерции $W$ точки $\ell$ в $G\left(K_{n} / k_{0}\right)$ является циклической $\ell$-группой, и $G\left(K_{n} / K_{n-1}\right) \subset W$. Так как $G\left(K_{n} / k\right) \cap G\left(K_{n} / K_{n-1}\right)=1$, то мы имеем $G\left(K_{n} / k\right) \cap W=1$, т.е. расширение $K_{n} / k$ не разветвлено над $\ell$.

Теперь, чтобы закончить доказательство леммы, мы должны рассмотреть случай, когда $\ell=2, n \geqslant 1$, и расширение $K_{n} / k$ разветвлено над точкой 2 . Пусть $W$ - подгруппа инерции точки 2 в $G\left(K_{n} / \mathbf{Q}\right)$. Тогда $W \cong\left(\mathbf{Z} / \ell^{m} \mathbf{Z}\right)^{*}$, поэтому $W=V \times \Delta$, где $\Delta \cong\{ \pm 1\}$ и $V \cong(1+4 \mathbf{Z}) /\left(1+2^{m} \mathbf{Z}\right) \cong \mathbf{Z} / 2^{n} \mathbf{Z}$. Так как $k \not K_{n-1}$, мы имеем $F \cap V=1$. Пусть $\sigma$ - единственный элемент порядка 2 в группе $V$ и $\tau$ - единственный отличный от единицы элемент группы $\Delta$, т.е. $\sigma\left(\zeta_{n}\right)=-\zeta_{n}$, $\tau\left(\zeta_{n}\right)=\zeta_{n}^{-1}$. Тогда $H=\{1, \sigma\}$ и условие $F \cap W=1$ означает, что либо $F \cap W=\{1, \tau\}$, либо $F \cap W=\{1, \sigma \tau\}$. Пусть $k_{1}$ - неподвижное поле относительно группы $F \cap W$. Тогда $K_{n} \supset k_{1} \supseteq k,\left[K_{n}: k_{1}\right]=2, G\left(K_{n} / k_{1}\right)=\{1, \tau\}$ или $\{1, \sigma \tau\}$ и $k_{1} / k$ не разветвлено над $\ell$.

Теперь нам нужно вычислить $\ell$-компоненты дифферент всех расширений, входящих в (6.5). В дальнейшем символ $\mathcal{D}$ будет обозначать $\ell$-компоненту дифференты. Хорошо известно, что $\mathcal{D}_{K_{n} / K_{n-1}}=(2)$. Если $G\left(K_{n} / k_{1}\right)=\{1, \tau\}$, то $\operatorname{Sp}_{K_{n} / k_{1}}\left(\zeta_{n}^{i}\right)=\zeta_{n}^{i}+\zeta_{n}^{-i}$. Положим $\pi=1-\zeta_{n-1}$. Поскольку $\operatorname{Sp}_{K_{n} / k_{1}}\left(\zeta_{n}\right)=$ $\zeta_{n}+\zeta_{n}^{-1} \equiv \zeta_{n}^{-1}\left(1-\zeta_{n-1}\right)(\bmod 2)$, мы получаем, что $\operatorname{Sp}_{K_{n} / k_{1}}\left(\mathcal{O}_{K_{n}}\right)=\pi \mathcal{O}_{k_{1}}$, 
т.е. $\operatorname{Sp}_{K_{n} / k_{1}}\left(\pi^{-1} \mathcal{O}_{K_{n}}\right)=\mathcal{O}_{k_{1}}$. Учитьвая, что $\operatorname{Sp}_{K_{n} / k_{1}}\left(\left(1-\zeta_{n}\right) \pi^{-2}\right) \notin \mathcal{O}_{k_{1}}$, мы получаем $\mathcal{D}_{K_{n} / k_{1}}=(\pi)$. Расширение $k_{1} / k$ не разветвлено над $\ell$, поэтому $\mathcal{D}_{K_{n} / k}=\mathcal{D}_{K_{n} / k_{1}}=(\pi)$ и $\nu_{\ell}\left(\mathcal{D}_{K_{n}} / k\right)=2^{-n}$. Если $G\left(K_{n} / k_{1}\right)=\{1, \sigma \tau\}$, то, рассуждая, как и выше, и полагая $\pi=\zeta_{n}-\zeta_{n}^{-1}$, мы снова получаем, что $\nu_{\ell}\left(\mathcal{D}_{K_{n} / k}\right)=2^{-n}$.

В силу мультипликативного свойства дифференты мы имеем

$$
\mathcal{D}_{K_{n} / k} \cdot \mathcal{D}_{k / k_{0}}=\mathcal{D}_{K_{n} / K_{n-1}} \cdot \mathcal{D}_{K_{n-1} / k_{0}} \cdot
$$

Мы уже вычислили $\mathcal{D}_{K_{n} / k}$. Хорошо известно, что $\mathcal{D}_{K_{n} / K_{n-1}}=(2)$. Заметим, что точки, лежащие над 2 , разветвлены в расширении $k / k_{0}$ (поскольку $K_{n}=k \times$ $\left.K_{n-1}\right)$, следовательно, они разветвлены и в расширении $K_{n-1} / k_{0}$. Тогда, если $n \geqslant 2$, то, рассуждая, как и вьше, мы получаем, что $\nu_{\ell}\left(\mathcal{D}_{K_{n-1}} / k_{0}\right)=2^{1-n}$. Если $n=1$, то расширение $k_{0} / \mathbf{Q}$ не разветвлено над точкой 2 . Тогда $\mathcal{D}_{K_{0} / k_{0}}=(2)$ и $\nu_{\ell}(\pi)=\frac{1}{2}$. Итак, в любом случае мы имеем $\nu_{\ell}\left(\mathcal{D}_{k / k_{0}}\right)=\nu_{\ell}(2 \pi)$. Следовательно, $\operatorname{Sp}_{k / k_{0}}\left(\widetilde{\mathcal{O}}_{k}\right)=2 \widetilde{\mathcal{O}}_{k_{0}}$

Если $x \in \widetilde{\mathcal{O}}_{k} \backslash \alpha(\pi) \widetilde{\mathcal{O}}_{k}$, то мы можем представить $x$ в виде $x=x_{1}+x_{2}$, где $x_{1} \in \widetilde{\mathcal{O}}_{k_{0}}^{*}$ и $x_{2} \in \alpha(\pi) \widetilde{\mathcal{O}}_{k}$. Пусть элемент $\pi_{0} \in k_{0}$ является локальным параметром в любой точке $v \mid \ell$ поля $k_{0}$. Тогда $\alpha(\pi) \widetilde{\mathcal{O}}_{k}=\alpha\left(\pi^{-1} \pi_{0}\right) \widetilde{\mathcal{O}}_{k}$, следовательно, имеют место включения $\operatorname{Sp}_{H}\left(x_{2}\right) \in \operatorname{Sp}_{H}\left(\alpha\left(\pi^{-1} \pi_{0}\right) \widetilde{\mathcal{O}}_{k}\right) \subset \alpha\left(2 \pi_{0}\right) \widetilde{\mathcal{O}}_{k}$, поскольку $\nu_{\ell}\left(\mathcal{D}_{k / k_{0}}\right)=\nu_{\ell}(2 \pi)$. С другой стороны, $\operatorname{Sp}_{H}\left(x_{1}\right)=2 x_{1} \notin \alpha\left(2 \pi_{0} \widetilde{\mathcal{O}}_{k_{0}}\right)$. Следовательно, $\operatorname{Sp}_{H}(x) \neq 0$, т.е. $x \notin \mathbf{B}_{k}$, откуда следует, что $\mathbf{B}_{k} \subset \alpha(\pi) \widetilde{\mathcal{O}}_{k}=$ $\operatorname{Sp}_{K_{n} / k}\left(\widetilde{\mathcal{O}}_{K_{n}}\right)$. Если $a \in \mathbf{B}_{k}$ и $a=\operatorname{Sp}_{F}(b), b \in \widetilde{\mathcal{O}}_{K_{n}}$, то согласно (6.6) мы имеем $b=b_{1}+b_{2}$, где $b_{1} \in \mathbf{B}_{K_{n}}, b_{2} \in \widetilde{\mathcal{O}}_{K_{n-1}}$. Тогда

$$
0=\operatorname{Sp}_{H}(a)=\operatorname{Sp}_{H} \operatorname{Sp}_{F}\left(b_{1}+b_{2}\right)=\operatorname{Sp}_{F} \operatorname{Sp}_{H}\left(b_{1}\right)+\operatorname{Sp}_{F} \operatorname{Sp}_{H}\left(b_{2}\right)=\operatorname{Sp}_{F}\left(2 b_{2}\right) .
$$

Следовательно, $\operatorname{Sp}_{F}\left(b_{2}\right)=0$ и $a=\operatorname{Sp}_{F}\left(b_{1}\right)$, т.е. отображение $\operatorname{Sp}_{F}: \mathbf{B}_{K_{n}} \rightarrow \mathbf{B}_{k}$ в (6.7) эпиморфно. Это означает, что $\mathbf{B}_{k}$ является циклическим $\overline{\mathcal{O}}[G]$-модулем, поэтому $\mathbf{B}_{k} \cong \mathbf{R}_{k} / \mathbf{R}_{k}^{H}$. Этим наша лемма доказана.

ЛЕмма 6.3. Пусть $k, k_{0}$ и $H$ те же, что и в (6.5). Положим $\mathbf{N}_{k}:=(1-$ $\left.\ell^{-1} \mathrm{Sp}_{H}\right) \mathbf{R}_{k} \subset \ell^{-1} \mathbf{R}_{k}$. Тогда

$$
\left(\mathbf{R}_{k, \varphi}: \widetilde{\mathcal{O}}_{k, \varphi}\right)=\left(\mathbf{N}_{k, \varphi}: \mathbf{B}_{k, \varphi}\right)\left(\mathbf{R}_{k, \varphi}: \widetilde{\mathcal{O}}_{k, \varphi}\right) \quad \text { для любого } \varphi \in \Phi .
$$

ДоКАЗАТЕЛЬСТво. Обозначим через $\mathbf{M}_{k}$ ядро отображения следа $\mathrm{Sp}_{H}: \mathbf{R}_{k} \rightarrow \mathbf{R}_{k_{0}}$. Тогда для заданного $\varphi \in \Phi$ мы имеем коммутативную диаграмму с точными строками

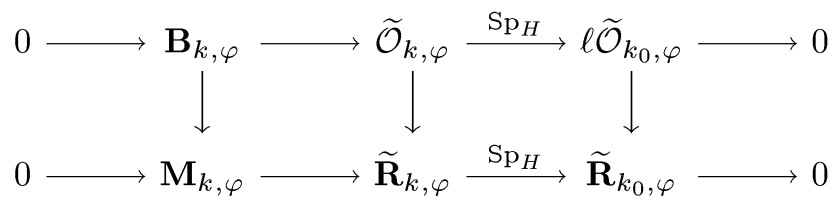

в которой вертикальные стрелки являются естественными вложениями.

Из (6.8) следует

$$
\left(\mathbf{R}_{k, \varphi}: \widetilde{\mathcal{O}}_{k, \varphi}\right)=\left(\mathbf{M}_{k, \varphi}: \mathbf{B}_{k, \varphi}\right)\left(\mathbf{R}_{k_{0}, \varphi}: \ell \widetilde{\mathcal{O}}_{k_{0}, \varphi}\right) .
$$


Поскольку $\mathbf{R}_{k} \cong \overline{\mathcal{O}}[G]$ как $\overline{\mathcal{O}}[G]$-модуль, отображение $\mathrm{Sp}_{H}: \mathbf{R}_{k} \rightarrow \mathbf{R}_{k_{0}}$ индуцирует изоморфизм $\mathbf{N}_{k} / \mathbf{M}_{k} \cong \mathbf{R}_{k_{0}} / \ell \mathbf{R}_{k_{0}}$. Следовательно, $\left(\mathbf{N}_{k, \varphi}: \mathbf{M}_{k, \varphi}\right)=\left(\mathbf{R}_{k_{0}, \varphi}:\right.$ $\left.\ell \mathbf{R}_{k_{0}, \varphi}\right)$, и тогда из $(6.9)$ следует, что

$$
\begin{aligned}
\left(\mathbf{R}_{k, \varphi}: \widetilde{\mathcal{O}}_{k, \varphi}\right) & =\left(\mathbf{M}_{k, \varphi}: \mathbf{B}_{k, \varphi}\right)\left(\mathbf{R}_{k_{0}, \varphi}: \ell \mathbf{R}_{k_{0}, \varphi}\right)\left(\ell \mathbf{R}_{k_{0}, \varphi}: \ell \widetilde{\mathcal{O}}_{k_{0}, \varphi}\right) \\
& =\left(\mathbf{N}_{k, \varphi}: \mathbf{B}_{k, \varphi}\right)\left(\mathbf{R}_{k_{0}, \varphi}: \widetilde{\mathcal{O}}_{k_{0}, \varphi}\right) .
\end{aligned}
$$

Лемма 6.4. Пусть $L, M \subset \mathbf{A}_{k}-$ две решетки, которье порождают одно и то же $\overline{\mathbf{Q}}_{\ell}$-пространство $V \subseteq \mathbf{A}_{k}$. Пусть $L, M$ являются $\overline{\mathcal{O}}[G]$-модулями. Для $\chi \in \widehat{G}$ положим $L_{\chi}=e_{\chi} L, \quad M_{\chi}=e_{\chi} M$, где $e_{\chi}=|G|^{-1} \sum_{\sigma \in G} \bar{\chi}(\sigma) \sigma-$ идемпотент, соответствующий $\chi$. Если $L \cong M$ как $\overline{\mathcal{O}}[G]$-модули, то

$$
(L: M)=\prod_{\chi \in \widehat{G}}\left(L_{\chi}: M_{\chi}\right)
$$

и для любого $\varphi \in \Phi$

$$
\left(L_{\varphi}: M_{\varphi}\right)=\prod_{\substack{\chi \in \widehat{G} \\ \chi \mid \varphi}}\left(L_{\chi}: M_{\chi}\right),
$$

где мы полагаем $\left(L_{\varphi}: M_{\varphi}\right)=1$ в случае, когда $L_{\varphi}=M_{\varphi}=0$.

ДокаЗАтЕльство. Положим $L^{\prime}=\bigoplus_{\chi \in \widehat{G}} L_{\chi}, M^{\prime}=\bigoplus_{\chi \in \widehat{G}} M_{\chi}$. Если отображение $f: L \cong M$ является $\overline{\mathcal{O}}[G]$-изоморфизмом, то $f$ индуцирует изоморфизмы $L^{\prime} \cong M^{\prime}$ и $L^{\prime} / L \cong M^{\prime} / M$. Тогда

$$
(L: M)=\left(L: L^{\prime}\right)\left(L^{\prime}: M^{\prime}\right)\left(M^{\prime}: M\right) .
$$

Учитывая, что $\left(L: L^{\prime}\right)^{-1}=\left(M^{\prime}: M\right)$ и $\left(L^{\prime}: M^{\prime}\right)=\prod_{\chi \in \widehat{G}}\left(L_{\chi}: M_{\chi}\right)$, мыполучаем первое утверждение леммы. Второе утверждение может быть доказано тем же способом. Лемма доказана.

Теперь мы можем закончить доказательство теоремы. Если $k$ не разветвлено над $\ell$, то равенство (6.3) было доказано в п. 1. Согласно п. 2 мы можем предполагать, что $\zeta_{0} \in k$ при $\ell \neq 2$. Итак, пусть $\zeta_{0} \in k$ и $k$ имеет кондуктор $N=\ell^{m} d$, где $m \geqslant 2$. Пусть $k_{0}$ то же, что и в (6.5). Поскольку $k_{0}$ имеет кондуктор $\ell^{m_{0}} d$, где $m_{0}=m-1\left(m_{0}=m-2=0\right.$ при $\left.\ell=m=2\right)$, мы можем предполагать, что (6.3) справедливо для $k_{0}$. Тогда согласно лемме 6.3 для заданного $\varphi \in \Phi$ мы имеем

$$
\left(\mathbf{R}_{k, \varphi}: \widetilde{\mathcal{O}}_{k, \varphi}\right)=\left(\mathbf{N}_{k, \varphi}: \mathbf{B}_{k, \varphi}\right)\left(\mathbf{R}_{k_{0}, \varphi}: \widetilde{\mathcal{O}}_{k_{0}, \varphi}\right),
$$

где по предположению индукции

$$
\nu_{\ell}\left(\left(\mathbf{R}_{k_{0}, \varphi}: \widetilde{\mathcal{O}}_{k_{0}, \varphi}\right)\right)=\nu_{\ell}\left(\prod_{\substack{\chi \in G\left(k_{0} / \mathbf{Q}\right) \\ \chi \mid \varphi}} G_{\bar{\chi}}\right)
$$

Для $\chi \in G \widehat{\left(k_{0} / \mathbf{Q}\right)}$ пусть $\chi^{\prime}$ обозначает характер $\chi$, рассматриваемый как характер групшы $G$. В этом случае $\chi \mid \varphi$ тогда и только тогда, когда $\chi^{\prime} \mid \varphi$, при этом $g_{\bar{\chi}}=g_{\overline{\chi^{\prime}}}$. Таким образом, мы имеем

$$
\prod_{\substack{\chi \in G\left(k_{0} / \mathbf{Q}\right) \\ \chi \mid \varphi, \chi \neq 1}} g_{\bar{\chi}}=\prod_{\substack{\chi \in \widehat{G}, \chi|\varphi \\ \chi|_{H}=1, \chi \neq 1}} g_{\bar{\chi}}
$$


Из леммы 6.2 и определения модуля $\mathbf{N}_{k}$ следует, что $\mathbf{N}_{k} \cong \mathbf{B}_{k}$ как $\overline{\mathcal{O}}[G]$-модуль. Тогда из леммы 6.4 мы получаем

$$
\left(\mathbf{N}_{k, \varphi}: \mathbf{B}_{k, \varphi}\right)=\prod_{\substack{\chi \in \widehat{G} \\ \chi \mid \varphi}}\left(\mathbf{N}_{k, \chi}: \mathbf{B}_{k, \chi}\right) .
$$

Заметим, что $\mathrm{Sp}_{H}$ аннулирует $\mathbf{N}_{k}$ и $\mathbf{B}_{k}$. Следовательно, если $\left.\chi\right|_{H}=1$, то $\mathbf{N}_{k, \chi}=\mathbf{B}_{k, \chi}=0$. Поэтому мы можем переписать (6.13) в виде

$$
\left(\mathbf{N}_{k, \varphi}: \mathbf{B}_{k, \varphi}\right)=\prod_{\substack{\chi \in \widehat{G} \\ \chi|\varphi, \chi|_{H} \neq 1}}\left(\mathbf{N}_{k, \chi}: \mathbf{B}_{k, \chi}\right) .
$$

Чтобы вычислить индекс $\left(\mathbf{N}_{k, \chi}: \mathbf{B}_{k, \chi}\right)$, мы сначала вычислим индекс $\left(\mathbf{N}_{K_{n}, \chi}\right.$ : $\left.\mathbf{B}_{K_{n}, \chi}\right)$, где мы рассматриваем $\chi$ как характер группы $G\left(K_{n} / \mathbf{Q}\right)$. Если $\left.\chi\right|_{H} \neq 1$, то $e_{\chi} \mathbf{N}_{K_{n}}=e_{\chi} \mathbf{R}_{K_{n}}=\overline{\mathcal{O}} e_{\chi} f_{1}$, где $f_{1}=(1,0, \ldots, 0) \in \mathbf{R}_{K_{n}}$ - образуюшая $\overline{\mathcal{O}}\left[G\left(K_{n} / \mathbf{Q}\right)\right]$-модуля $\mathbf{R}_{K_{n}}$, определенная в начале параграфа.

Мы знаем, что $\mathbf{B}_{K_{n}}$ порождается над $\overline{\mathcal{O}}$ всеми элементами вида $\alpha\left(\varepsilon \zeta_{n}^{i}\right)$, где $\varepsilon \in \mu_{d}$ и $i \not \equiv 0(\bmod \ell)$. Заметим, что $\varepsilon \zeta_{n}^{i}$ является первообразным корнем из единицы степени $\ell^{m} d_{0}$ для некоторого $d_{0} \mid d$. Если $\left.\chi\right|_{H} \neq 1$, то $\chi$ имеет кондуктор $f_{\chi}=\ell^{m} d_{1}$ для некоторого $d_{1} \mid d$. Пусть $\xi-$ первообразный корень из единищы степени $d_{0} \ell^{m}$ для некоторого $d_{0} \mid d$. Тогда

$$
e_{\chi} \alpha(\xi)=\sum_{\sigma \in G_{n}} \bar{\chi}(\sigma) \sigma(\xi) e_{\chi} f_{1}
$$

где $G_{n}=G\left(K_{n} / \mathbf{Q}\right)$.

Пусть $H(\chi)$ - ядро естественной проекции

$$
G\left(K_{n} / \mathbf{Q}\right)=\left(\mathbf{Z} / \ell^{m} d \mathbf{Z}\right)^{*} \rightarrow\left(\mathbf{Z} / f_{\chi} \mathbf{Z}\right)^{*}
$$

а $H(\xi)$ - ядро естественной проекции

$$
G\left(K_{n} / \mathbf{Q}\right)=\left(\mathbf{Z} / \ell^{m} d \mathbf{Z}\right)^{*} \rightarrow\left(\mathbf{Z} / \ell^{m} d_{0} \mathbf{Z}\right)^{*},
$$

т.е. $H(\chi) \cong \operatorname{Ker}\left((\mathbf{Z} / d \mathbf{Z})^{*} \rightarrow\left(\mathbf{Z} / d_{1} \mathbf{Z}\right)^{*}\right)$ и $H(\xi) \cong \operatorname{Ker}\left((\mathbf{Z} / d \mathbf{Z})^{*} \rightarrow\left(\mathbf{Z} / d_{0} \mathbf{Z}\right)^{*}\right)$. Если $d_{1} \backslash d_{0}$, то, полагая $G_{n} / H(\chi) \cap H(\xi)=S, H(\xi) / H(\chi) \cap H(\xi)=T \neq 1$, мы получаем

$$
\begin{aligned}
\sum_{\sigma \in G_{n}} \bar{\chi}(\sigma) \sigma(\xi) & =|H(\chi) \cap H(\xi)| \sum_{\sigma \in S} \bar{\chi}(\sigma) \sigma(\xi) \\
& =|H(\chi) \cap H(\xi)| \sum_{\sigma \in \overline{S / T}} \sum_{\tau \in T} \bar{\chi}(\sigma \tau) \sigma \tau(\xi)
\end{aligned}
$$

где $\overline{S / T} \subset S$-некоторая система представителей для $S / T$. Мы имеем $\sigma \tau(\xi)=\sigma(\xi)$ для любого $\tau \in T$ и $\sum_{\tau \in T} \bar{\chi}(\sigma \tau)=\bar{\chi}(\sigma) \sum_{\tau \in T} \bar{\chi}(\tau)$. Заметим, что $\left.\chi\right|_{H(\xi)} \neq 1$, поэтому $\left.\chi\right|_{T} \neq 1$ и $\sum_{\tau \in T} \bar{\chi}(\tau)=0$. Таким образом, если $d_{1} \chi d_{0}$, то $\sum_{\sigma \in G_{n}} \bar{\chi}(\sigma) \sigma(\xi)=0$. 
Если $d_{1} \mid d_{0}$, то $H(\xi) \subseteq H(\chi)$ и, полагая $A=H(\chi) / H(\xi), B=G_{n} / H(\xi)$, $C=G_{n} / H(\chi)$, мы получаем

$$
\sum_{\sigma \in G_{n}} \bar{\chi}(\sigma) \sigma(\xi)=|H(\xi)| \sum_{\sigma \in B} \bar{\chi}(\sigma) \sigma(\xi)=\sum_{\sigma \in C} \bar{\chi}(\sigma) \sigma\left(\operatorname{Sp}_{A}(\xi)\right) .
$$

Пусть $d_{0} / d_{1}=q_{1}^{s_{1}}, \ldots, q_{r}^{s_{r}}$ - разложение числа $d_{0} / d_{1}$ на простые множители. Хорошо известно, что для первообразного корня из единицы $\mu$ степени $p^{n}$ для некоторого простого $p$ вьполняются равенства

$$
\operatorname{Sp}_{\mathbf{Q}(\mu) / \mathbf{Q}}(\mu)=\left\{\begin{array}{lll}
-1 & \text { при } & n=1 \\
0 & \text { при } & n \geqslant 1 .
\end{array}\right.
$$

Более того, если $n \geqslant 1, \operatorname{ro~}_{\mathbf{S p}_{\mathbf{Q}}(\mu) / \mathbf{Q}\left(\mu^{p}\right)}(\mu)=0$. Таким образом, мы имеем

$$
\operatorname{Sp}_{A}(\xi)= \begin{cases}0 & \text { при } s_{i} \geqslant 1 \text { для некоторого } i=1, \ldots, r, \\ 0 & \text { при } d_{1} \equiv 0\left(\bmod q_{i}\right) \text { для некоторого } i=1, \ldots, r \\ \pm \xi_{1} & \text { при } s_{1}=\cdots=s_{r}=1 \quad \text { и }\left(d_{1}, d_{0} / d_{1}\right)=1,\end{cases}
$$

где $\xi_{1}$ - некоторый первообразньй корень из единицы степени $\ell^{m} d_{1}$.

Итак, в любом случае мы получаем

$$
\sum_{\sigma \in G_{n}} \bar{\chi}(\sigma) \sigma(\xi)=|H(\xi)| \eta g_{\bar{\chi}}
$$

где $\eta$ - некоторый корень из единищы или $\eta=0$. Следовательно, $e_{\chi} \mathbf{B}_{K_{n}} \subseteq$ $\overline{\mathcal{O}} g_{\bar{\chi}} e_{\chi} f_{1}$.

Пусть $p_{1}, \ldots, p_{r}$ - все простые делители $d / d_{1}$ такие, что $\left(p_{i}, d_{1}\right)=1$ для $i=$ $1, \ldots, r$. Положим $d_{0}=d_{1} p_{1} \cdots p_{r}$ и $\xi=\zeta_{n} \varepsilon_{d_{1}} \varepsilon_{p_{1}} \cdots \varepsilon_{p_{r}}$, где $\varepsilon_{n}$ - некоторый первообразный корень из единицы степени $n$. Тогда $\xi$ - первообразный корень из единицы степени $\ell^{m} d_{0}$, и при нашем выборе $d_{0}$ мы получаем, что $\operatorname{Sp}_{A}(\xi)$ в $(6.15)$ задается формулой

$$
\operatorname{Sp}_{A}(\xi)=(-1)^{r} \xi_{1}
$$

где $\xi_{1}$ - некоторый первообразный корень из единицы степени $\ell^{m} d_{1}$. Заметим, что любой простой делитель $p$ числа $d$ делит и $d_{0}$, следовательно, $p$ делит порядок групшы $H(\xi)$, только если $p \mid d$. Поэтому $|H(\xi)|$ взаимно прост с $\ell$. Таким образом, множитель $|H(\xi)| \eta$ является единищей в $\overline{\mathcal{O}}$. Это доказывает, что

$$
e_{\chi} \mathbf{B}_{K_{n}}=\overline{\mathcal{O}} g_{\bar{\chi}} e_{\chi} f_{1} \quad \text { и } \quad\left(\mathbf{N}_{K_{n}, \chi}: \mathbf{B}_{K_{n}, \chi}\right)=g_{\bar{\chi}} .
$$

Теперь если $\chi \in \widehat{G}$, то мы можем рассматривать $\chi$ как характер группы $G_{n}=$ $G\left(K_{n} / \mathbf{Q}\right)$ такой, что $\left.\chi\right|_{F}=1$. Поскольку в силу леммы 6.2 мы имеем эпиморфизмы

$$
\mathrm{Sp}_{F}: \mathbf{N}_{K_{n}, \chi} \rightarrow \mathbf{N}_{k, \chi}, \quad \mathrm{Sp}_{F}: \mathbf{B}_{K_{n}, \chi} \rightarrow \mathbf{B}_{k, \chi}
$$

и все рассматриваемые модули имеют ранг 1 над $\overline{\mathcal{O}}$, мы получаем, что отображения (6.17) являются изоморфизмами. Следовательно,

$$
\left(\mathbf{N}_{k, \chi}: \mathbf{B}_{k, \chi}\right)=\left(\mathbf{N}_{K_{n}, \chi}: \mathbf{B}_{K_{n}, \chi}\right)=g_{\bar{\chi}}
$$

где для $\chi=1$ мы полагаем по определению $g_{\bar{\chi}}=1$. Сопоставляя $(6.10),(6.11)$, $(6.12),(6.14)$ и (6.18), мы получаем (6.3). Этим наша теорема полностью доказана. 


\section{$\S 7$. Некоторые индексы}

Пусть $k$-вешественное абелево поле и $\mathbf{A}_{k}, \mathbf{R}_{k}, \widetilde{\mathcal{O}}_{k}$ имеют тот же смысл, что и в $\S 6$. Как и во введении, мы полагаем $\mathcal{A}(k)=\prod_{v \mid \ell}\left(U\left(k_{v}\right) / \mu\left(k_{v}\right)\right)$. Для любой точки

$v \mid \ell$ имеется инъективное отображение $\log _{v}: U\left(k_{v}\right) / \mu\left(k_{v}\right) \rightarrow k_{v}$, определяемое при помоши $\ell$-адического логарифма. Беря прямое произведение отображений $\log _{v}$ по всем $v \mid \ell$, мы получаем инъективное отображение

$$
\log : \mathcal{A}(k) \hookrightarrow \prod_{v \mid \ell} k_{v}
$$

Отображение (7.1) является гомоморфизмом $\mathbf{Z}_{\ell}[G]$-модулей, где $G=G(k / \mathbf{Q})$, и действие $G$ на $\mathcal{A}(k)$ и $\prod_{v \mid \ell} k_{v}$ определяется при помощи отождествления $\prod_{v \mid \ell} k_{v} \cong$ $\mathbf{Q}_{\ell} \otimes_{\mathbf{Q}} k$. Естественное включение $\mathbf{Q}_{\ell} \hookrightarrow \overline{\mathbf{Q}}_{\ell}$ индуцирует включение

$$
i: \prod_{v \mid \ell} k_{v} \cong \mathbf{Q}_{\ell} \otimes_{\mathbf{Q}} k \hookrightarrow \overline{\mathbf{Q}}_{\ell} \otimes_{\mathbf{Q}} k=\mathbf{A}_{k} .
$$

Полагая $\log =i \cdot \log$, мы получаем вложение $\mathbf{Z}_{\ell}[G]$-модулей

$$
\log : \mathcal{A}(k) \longrightarrow \mathbf{A}_{k}
$$

Через $\widetilde{\mathcal{A}}(k)$ мы будем обозначать $\overline{\mathcal{O}}[G]$-подмодуль $\overline{\mathcal{O}} \cdot \log (\mathcal{A}(k))$ модуля $\mathbf{A}_{k}$.

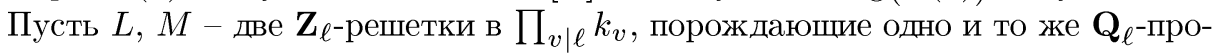
странство $V \subseteq \prod_{v \mid \ell} k_{v}$. Тогда,как и в [15], мы определяем индекс $(L: M)$ как

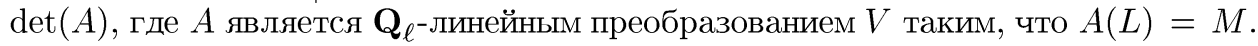
Индекс $(L: M)$ определен однозначно с точностью до умножения на произвольньй элемент из $\mathbf{Z}_{\ell}^{*}$. Если $L \supseteq M$ и индекс $(L: M)$ существует, то обычный индекс $[L: M]$ конечен и

$$
\nu_{\ell}((L: M))=\nu_{\ell}([L: M]) .
$$

Если мы положим $\widetilde{L}=\overline{\mathcal{O}} \cdot i(L), \widetilde{M}=\overline{\mathcal{O}} \cdot i(M)$, то индекс $(\widetilde{L}: \widetilde{M})$, определенный в $\S 6$, существует тогда и только тогда, когда существует индекс $(L: M)$, и в этом случае

$$
\nu_{\ell}((L: M))=\nu_{\ell}((\widetilde{L}: \widetilde{M})) .
$$

Пусть $\widetilde{\mathcal{O}}_{k}$ имеет тот же смысл, что и в $\S 6$.

ПрЕДЛОЖЕНИЕ 7.1. Индекс $\left(\widetilde{\mathcal{A}}(k): \widetilde{\mathcal{O}}_{k}\right)$ существует $u$

$$
\left(\widetilde{\mathcal{O}}_{k}: \widetilde{\mathcal{A}}(k)\right)=|P(k)| \cdot|Y(k)|
$$

әде $P(k)=\prod_{v \mid \ell} \bar{k}_{v}, \bar{k}_{v}$ - поле вычетов поля $k_{v}$, a $Y(k)$ имеет тот же смицсл, что и в теореме 5.1 .

Для любого $\varphi \in \Phi$ справедливо равенство

$$
\left(\widetilde{\mathcal{O}}_{k, \varphi}: \widetilde{\mathcal{A}}(k)_{\varphi}\right)=\left|P(k)_{\varphi}\right| \cdot\left|Y(k)_{\varphi}\right| .
$$


ДокАЗАТЕЛЬСТво. Пусть $\mathcal{O}_{v}=\mathcal{O}\left(k_{v}\right)$ - кольцо целых поля $k_{v}$. Положим $\mathcal{O}_{\ell}=\prod_{v \mid \ell} \mathcal{O}_{v}$. Тогда в силу (7.3) мы имеем

$$
\left(\widetilde{\mathcal{O}}_{k}: \widetilde{\mathcal{A}}(k)\right)=\left(\mathcal{O}_{\ell}: \log \mathcal{A}(k)\right) \quad \text { и } \quad\left(\widetilde{\mathcal{O}}_{k, \varphi}: \widetilde{\mathcal{A}}(k)_{\varphi}\right)=\left(\mathcal{O}_{\ell, \varphi}:(\log \mathcal{A}(k))_{\varphi}\right) .
$$

Пусть $\pi_{v}$ - локальный параметр поля $k_{v}$. Положим

$$
U^{(i)}\left(k_{v}\right)=\left\{x \in U\left(k_{v}\right) \mid x \equiv 1 \quad\left(\bmod \pi_{v}^{i}\right)\right\}
$$

Хорошо известно, что для всех достаточно больших $i \quad \ell$-адический логарифм $\log _{v}$ устанавливает изоморфизм $\log _{v}: U^{(i)}\left(k_{v}\right) \cong \pi_{v}^{i} \mathcal{O}_{v}$. Поэтому отображение $(7.1)$ индуцирует для любого такого $i$ точную последовательность

$$
0 \longrightarrow Y(k) \longrightarrow \prod_{v \mid \ell}\left(U^{(1)}\left(k_{v}\right) / U^{(i)}\left(k_{v}\right)\right) \stackrel{\log ^{(i)}}{\longrightarrow} \prod_{v \mid \ell}\left(k_{v} / \pi_{v}^{i} \mathcal{O}_{v}\right) .
$$

Поскольку $\prod_{v \mid \ell}\left(U^{(i)}\left(k_{v}\right) / U^{(i+1)}\left(k_{v}\right)\right) \cong P(k)$ для любого $i \geqslant 1$ и $\prod_{v \mid \ell}\left(\pi_{v}^{i} \mathcal{O}_{v} /\right.$ $\left.\pi_{v}^{i+1} \mathcal{O}_{v}\right) \cong P(k)$ для любого $i \geqslant 0$, то мы получаем из (7.4)

$$
\left(\mathcal{O}_{\ell}: \log \mathcal{A}(k)\right)=|Y(k)| \cdot|P(k)| .
$$

Это доказывает первое утверждение предложения. Второе утверждение доказывается аналогично. Предложение доказано.

Как и в $[15]$, мы полагаем для любого $G$-модуля $A$

$$
A_{0}=\{a \in A \mid s(G) a=0\}
$$

где $s(G)=\sum_{\sigma \in G} \sigma$. Мы имеем $\left(A_{\varphi}\right)_{0}=\left(A_{0}\right)_{\varphi}$ для любого $\varphi \in \Phi$, и при $\varphi \neq \varphi_{0}$ справедливо равенство $\left(A_{\varphi}\right)_{0}=A_{\varphi}$. Поэтому мы можем просто писать $A_{\varphi, 0}$.

Пусть $k$ имеет кондуктор $N=\ell^{m} d$, где $(d, \ell)=1$. Положим $K_{n}=\mathbf{Q}\left(\mu_{d}, \zeta_{n}\right)$. Чтобы сформулировать наши дальнейшие результаты, напомним, что, как было показано при доказательстве леммы 6.2 , в случае $\ell=2$ имеются три возможности:

(А) расширение $K_{n} / k$ не разветвлено над $\ell$;

(B) $G\left(K_{n} / k\right) \cap G\left(K_{n} / K_{-1}\right)=\{1, \tau\}$;

(C) $G\left(K_{n} / k\right) \cap G\left(K_{n} / K_{-1}\right)=\{1, \sigma \tau\}$;

где $\sigma, \tau$ были определены в доказательстве леммы 6.2. Заметим, что в случаях (B), (C) любая точка $v \mid \ell$ имеет индекс ветвления 2 в расширении $K_{n} / k$.

ПРЕДЛОЖЕНИЕ 7.2. Справедливы равенства

$$
\begin{aligned}
\left(\widetilde{\mathcal{O}}_{k, 0}: \widetilde{\mathcal{A}}(k)_{0}\right) & =\ell^{a}|P(k)| \cdot|Y(k)|, \\
\left(\widetilde{\mathcal{O}}_{k, 0, \varphi_{0}}: \widetilde{\mathcal{A}}(k)_{0, \varphi_{0}}\right) & =\ell^{a}\left|P(k)_{\varphi_{0}}\right| \cdot\left|Y(k)_{\varphi_{0}}\right|,
\end{aligned}
$$

где $a=-1$ при $\ell \neq 2$. Если $\ell=2$, то мы имеем $a=-2$ при $m=0$, а при $m>0$ мии имеем

$$
a= \begin{cases}-1 & \text { для } k \text { muna }(\mathrm{A}),(\mathrm{C}) \\ -2 & \text { для } k \text { muna }(\mathrm{B}) .\end{cases}
$$


ДокаЗАтеЛЬСтво. Пусть $L, M$ - некоторые $\overline{\mathcal{O}}$-решетки в $\mathbf{A}_{k}$ (соответственно

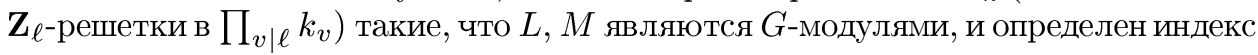
$(L: M)$. Тогда оба индекса $\left(L_{0}: M_{0}\right)$ и $\left(\operatorname{Sp}_{G}(L): \operatorname{Sp}_{G}(M)\right)$ определены и

$$
(L: M)=\left(L_{0}: M_{0}\right)\left(\operatorname{Sp}_{G}(L): \operatorname{Sp}_{G}(M)\right) .
$$

Действительно, в [15, лемма 6.1] аналогичное утверждение было доказано для $\mathbf{Z}$-решеток в $\mathbf{Q}[G]$. Те же аргументы показывают, что (7.5) справедливо также для

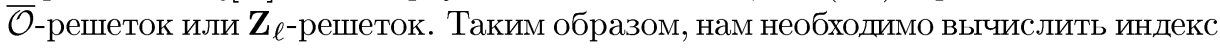

$$
\ell^{-a}=\left(\operatorname{Sp}_{G}\left(\widetilde{\mathcal{O}}_{k}\right): \operatorname{Sp}_{G}(\widetilde{\mathcal{A}}(k))\right)=\left(\operatorname{Sp}_{G}\left(\widetilde{\mathcal{O}}_{k, \varphi_{0}}\right): \operatorname{Sp}_{G}\left(\widetilde{\mathcal{A}}(k)_{\varphi_{0}}\right)\right) .
$$

Отметим, что согласно (7.3) мы имеем

$$
\left(\operatorname{Sp}_{G}\left(\widetilde{\mathcal{O}}_{k}\right): \operatorname{Sp}_{G}(\widetilde{\mathcal{A}}(k))\right)=\left(\operatorname{Sp}_{G}\left(\mathcal{O}_{\ell}\right): \operatorname{Sp}_{G}(\log \mathcal{A}(k))\right) .
$$

Если $G_{v}$ - подгруппа разложения некоторой точки $v \mid \ell$, то

$$
\left(\operatorname{Sp}_{G}\left(\mathcal{O}_{\ell}\right): \operatorname{Sp}_{G}(\log \mathcal{A}(k))\right)=\left(\operatorname{Sp}_{G_{v}}\left(\mathcal{O}\left(k_{v}\right)\right): \operatorname{Sp}_{G_{v}}\left(\log \bar{U}\left(k_{v}\right)\right)\right) .
$$

Пусть $T_{v} \neq 1$ - подгруппа инерции в группе $G_{v}$. Тогда

$$
\nu_{\ell}\left(\left|T_{v}\right|\right)=\left\{\begin{array}{lllll}
m-1 & \text { для } \ell \neq 2, & & \\
m-1 & \text { для } \ell=2 & \text { и } & k & \text { типа }(\mathrm{A}), \\
m-2 & \text { для } \ell=2 & \text { и } & k & \text { типа }(\mathrm{B}),(\mathrm{C}) .
\end{array}\right.
$$

Если $F_{v}=k_{v}^{T_{v}}$, то $F_{v} / \mathbf{Q}_{\ell}$ не разветвлено и $\operatorname{Sp}_{F_{v} / \mathbf{Q}_{\ell}}\left(\mathcal{O}_{F_{v}}\right)=\mathbf{Z}_{\ell}$. Пусть $\mathcal{D}_{k_{v} / \mathbf{Q}_{\ell}}-$ дифферента расширения $k_{v} / \mathbf{Q}_{\ell}$. Тогда, используя вычисления из доказательства леммы 6.2 , можно легко проверить, что в любом случае $\mathcal{D}_{k_{v} / \mathbf{Q}_{\ell}} \subseteq\left|T_{v}\right| \mathcal{O}\left(k_{v}\right)$, следовательно, $\operatorname{Sp}_{G_{v}}\left(\mathcal{O}\left(k_{v}\right)\right) \subseteq\left|T_{v}\right| \mathbf{Z}_{\ell}$. С другой стороны, $\operatorname{Sp}_{G_{v}}\left(\mathcal{O}\left(F_{v}\right)\right)=\left|T_{v}\right| \mathbf{Z}_{\ell}$. Таким образом, в любом случае, включая случай $T_{v}=1$, мы имеем $\operatorname{Sp}_{G_{v}}\left(\mathcal{O}\left(k_{v}\right)\right)=\left|T_{v}\right| \mathbf{Z}_{\ell}$.

Чтобы вычислить $\operatorname{Sp}_{G_{v}}\left(\log \bar{U}\left(k_{v}\right)\right)$, мы заметим, что $\operatorname{Sp}_{G_{v}}\left(\log \bar{U}\left(k_{v}\right)\right)=$ $\log N_{k_{v} / \mathbf{Q}_{\ell}}\left(U\left(k_{v}\right)\right)$. Согласно локальной теории полей классов мы имеем $\mathbf{Z}_{\ell}^{*} / N_{k_{v} / \mathbf{Q}_{\ell}}\left(U\left(k_{v}\right)\right) \cong T_{v}$. Учитывая, что $\log \left(\mathbf{Z}_{\ell}^{*}\right)=\ell \mathbf{Z}_{\ell}$ при $\ell \neq 2$ (соответственно $\ell^{2} \mathbf{Z}_{\ell}$ при $\left.\ell=2\right)$, мы получаем, что $\operatorname{Sp}_{G_{v}}\left(\log \bar{U}\left(k_{v}\right)\right)=\ell^{m} \mathbf{Z}_{\ell}$ при $\ell \neq 2$. Это доказы-

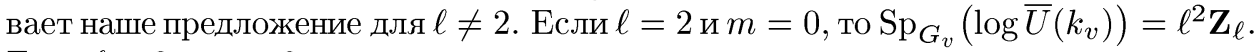
Если $\ell=2$ и $m>0$, то

$$
N_{k_{v} / \mathbf{Q}_{\ell}}\left(U\left(k_{v}\right)\right)= \begin{cases}1+\ell^{m} \mathbf{Z}_{\ell} & \text { для } k \text { типа (A), } \\ \{ \pm 1\} \times\left(1+\ell^{m} \mathbf{Z}_{\ell}\right) & \text { для } k \text { типа (B), } \\ \left\langle-\left(1+\ell^{m-1}\right)\right\rangle & \text { для } k \text { типа (C). }\end{cases}
$$

Следовательно,

$$
\operatorname{Sp}_{G_{v}}\left(\log \bar{U}\left(k_{v}\right)\right)= \begin{cases}\ell\left|T_{v}\right| \mathbf{Z}_{\ell} & \text { для } k \text { типа (A), } \\ \ell^{2}\left|T_{v}\right| \mathbf{Z}_{\ell} & \text { для } k \text { типа (B), } \\ \ell\left|T_{v}\right| \mathbf{Z}_{\ell} & \text { для } k \text { типа (C). }\end{cases}
$$

Предложение доказано. 
ПРЕДЛОЖЕНИЕ 7.3. Пусть $k$ имеет кондуктор $N=\ell^{m} d, \quad(d, \ell)=1 u$ $G=G(k / \mathbf{Q})$. Тогда

$$
\begin{gathered}
\nu_{\ell}\left(\left(\mathbf{R}_{k, 0}: \widetilde{O}_{k, 0}\right)\right)=-c+\nu_{\ell}\left(\prod_{\substack{\chi \in \widehat{G} \\
\chi \neq 1}} g_{\bar{\chi}}\right), \\
\nu_{\ell}\left(\left(\mathbf{R}_{k, \varphi_{0}, 0}: \widetilde{O}_{k, \varphi_{0}, 0}\right)\right)=-c+\nu_{\ell}\left(\prod_{\substack{\chi \in \widehat{G} \\
\chi \mid \varphi_{0}, \chi \neq 1}} g_{\bar{\chi}}\right),
\end{gathered}
$$

əде

$$
c= \begin{cases}0 & \text { для } m=0, \\ m-1 & \text { для } m>0 u \ell \neq 2, \\ m-1 & \text { для } m>0, \ell=2 \text { uk muna }(\mathrm{A}), \\ m-2 & \text { для } m>0, \ell=2 \text { uk muпa }(\mathrm{B}) \text { или }(\mathrm{C}) .\end{cases}
$$

Доказательство. Отображение $\mathrm{Sp}_{G}: \mathbf{A}_{k} \rightarrow \mathbf{A}_{\mathbf{Q}}$ отображает $\mathbf{R}_{k}$ на $\overline{\mathcal{O}}$. В доказательстве предложения 7.2 мы показали, что $\operatorname{Sp}_{G}\left(\mathcal{O}_{\ell}\right)=\ell^{c} \mathbf{Z}_{\ell}$, где $c$ определено, как и вьше. Следовательно, $\operatorname{Sp}_{G}\left(\widetilde{\mathcal{O}}_{k}\right)=\ell^{c} \overline{\mathcal{O}}$. Сопоставляя это с теоремой 6.1 и используя (7.5), мы получаем утверждение предложения.

Пусть $k$ будет, как и в предложении 7.3. Положим $K_{\infty}=\bigcup_{n=1}^{\infty} \mathbf{Q}\left(\mu_{d}, \zeta_{n}\right)$. Пусть $\tau: G_{\infty}=G\left(K_{\infty} / \mathbf{Q}\right) \rightarrow G$ - естественньй эпиморфизм, индуцированньй включением $k \subset K_{\infty}$.

ПРЕДЛОЖЕНИЕ 7.4. Пусть $k$ имеет кондуктор $\ell^{m} d$, как и выше, и $r$ константа, соответсвующая $\tau$, которая была определена в (2.5). Пусть $k_{s}-$ максимальное подполе поля $k_{\infty}$ такое, что расширение $k_{s} / k$ не разветвлено (напомним, что $\left.\left[k_{s}: k\right]=\ell^{s}\right)$. Если $m=0$, то $s=r=0$. Если $m>0$, то

$$
s= \begin{cases}m-1-r & \partial \bumpeq s \quad \ell \neq 2, \\ m-2-r & \partial \Omega я \ell=2 \text { uk muna }(\mathrm{A}),(\mathrm{B}), \\ m-3-r & \partial \Omega я \ell=2 \text { uk muna }(\mathrm{C}) .\end{cases}
$$

ДокАЗАТЕЛЬСтво. Пусть $\mathbf{Q}_{\infty}-$ круговое $\mathbf{Z}_{\ell}$-расширение поля $\mathbf{Q}$ и $\mathbf{Q}_{\infty}=$ $\bigcup_{i=1}^{\infty} \mathbf{Q}_{i}$, где $\left[\mathbf{Q}_{i}: \mathbf{Q}\right]=\ell^{i}$. Тогда $(2.5)$ показьвает, что $\mathbf{Q}_{r} \subseteq k, \mathbf{Q}_{r+1} \not k k$, другими словами, $k_{i}=k \cdot \mathbf{Q}_{r+i}$ для любого $i \geqslant 1$.

Если $m=0$, то $k \subseteq \mathbf{Q}\left(\mu_{d}\right)$. В этом случае все точки $v \mid \ell$ вполне разветвлены в расширении $k_{\infty} / k$, следовательно, мы имеем $r=s=0$, как и требуется.

Если $m>0$ и $\ell \neq 2$, то $K_{n} / k$ не имеет дикого ветвления. Следовательно, поле $k_{n-r}=K_{n} \cap k_{\infty}$ не имеет дикого ветвления над $k$, т.е. расширение $k_{n-r} / k$ не разветвлено. Поскольку $K_{n+1}=k_{n-r+1} K_{n}$, мы получаем, что поле $k_{n-r+1}$ разветвлено над $k$. Следовательно, в этом случае $s=n-r=m-1-r$. Если $\ell=2, m>0$ и поле $k$ имеет один из типов (A), (В), то, рассуждая, как и выше, мы получаем, что $s=n-r=m-2-r$.

Если $\ell=2, m>0$ и $k$-поле типа $(\mathrm{C})$, то $G\left(K_{n} / k\right) \cap G\left(K_{n} / K_{-1}\right)=\{1, \sigma \tau\}$. В этом случае подгруппы инерции точек $v \mid \ell$ в расширениях $G\left(k_{\infty} / \mathbf{Q}\right)$ и $G\left(K_{\infty} / \mathbf{Q}\right)$ изоморфны, следовательно, мы получаем, что $k_{n-r}=K_{n} \cap k_{\infty}$, и расширение 
$K_{n} / k_{n-r}$ не разветвлено. Поскольку расширение $K_{n} / k$ разветвлено и имеет индекс ветвления 2 относительно любой точки $v \mid \ell$, мы получаем, что расширение $k_{n-r} / k_{n-r-1}$ разветвлено, а расширение $k_{n-r-1} / k$ не имеет ветвления. Поэтому в этом случае $s=n-r-1=m-3-r$. Предложение доказано.

Для вешественного абелева поля $k$ диагональное вложение $U(k) \hookrightarrow \prod_{v \mid \ell} U\left(k_{v}\right)$ индуцирует вложение $\bar{U}(k)[\ell] \hookrightarrow \mathcal{A}(k)$. Поэтому мы можем рассматривать группу $\bar{U}(k)[\ell]$ как подгруппу группы $\mathcal{A}(k)$. Поскольку $N_{k / \mathbf{Q}}(\bar{U}(k)[\ell])=1$, мы получаем, что $\bar{U}(k)[\ell] \subseteq \mathcal{A}(k)_{0}$. Так как $\bar{U}(k)[\ell]$ и $\mathcal{A}(k)_{0}$ имеют одинаковый ранг над $\mathbf{Z}_{\ell}$, мы получаем, что $\bar{U}(k)[\ell]$ имеет конечньй индекс в $\mathcal{A}(k)_{0}$.

ТЕОРЕМА 7.1. Пусть $k$ - произвольное вещественное абелево поле $u$ $T_{\ell}\left(k_{\infty}\right)_{(0)}, H(k), U_{S}(k)$ имеют тот же смысл, что и в теореме 5.1. Через $\mathrm{Cl}(k)_{\ell}$ мы обозначаем $\ell$-компоненту группы классов поля $k$. Пусть $s-$ константа, определенная в предлодсении 7.4. Тогда

$$
\frac{\ell^{s}\left|T_{\ell}\left(k_{\infty}\right)_{(0)}\right|}{\left|\mathrm{Cl}(k)_{\ell}\right|}=\frac{\left[\mathcal{A}(k)_{0}: \bar{U}(k)[\ell]\right]}{\left[H(k): \widehat{U}_{S}(k)\right]} .
$$

Для любого характера $\varphi \in \Phi, \varphi \neq \varphi_{0}$, мы имеем

$$
\frac{\left|T_{\ell}\left(k_{\infty}\right)_{(0), \varphi}\right|}{\left|\operatorname{Cl}(k)_{\ell, \varphi}\right|}=\frac{\left[\mathcal{A}(k)_{\varphi}: \bar{U}(k)[\ell]_{\varphi}\right]}{\left[H(k)_{\varphi}: \widehat{U}_{S}(k)_{\varphi}\right]} .
$$

Eсли $\varphi=\varphi_{0}, m o$

$$
\frac{\ell^{s}\left|T_{\ell}\left(k_{\infty}\right)_{(0), \varphi_{0}}\right|}{\left|\operatorname{Cl}(k)_{\ell, \varphi_{0}}\right|}=\frac{\left[\mathcal{A}(k)_{0, \varphi_{0}}: \bar{U}(k)[\ell]_{\varphi_{0}}\right]}{\left[H(k)_{\varphi_{0}}: \widehat{U}_{S}(k)_{\varphi_{0}}\right]} .
$$

ДокАЗАТЕЛЬСТво. Положим $\mathrm{Cl}_{S}(k):=\mathrm{Cl}(k) / P_{S}(k)$, где $P_{S}(k)$ - подгруппа групшы $\mathrm{Cl}(k)$, порожденная всеми простыми дивизорами, лежашими над $\ell$. Пусть $\mathrm{Cl}_{S}(k)_{\ell}-\ell$-компонента группы $\mathrm{Cl}_{S}(k)$. Согласно теории полей классов имеется естественный изоморфизм $\mathrm{Cl}_{S}(k)_{\ell} \cong G\left(k_{S, \ell} / k\right)$, где $G\left(k_{S, \ell} / k\right)$ - группа Галуа максимального абелева неразветвленного $\ell$-расширения поля $k$, в котором все точки $v \mid \ell$ вполне распадаются. Тогда имеются естественные отображения

$$
T_{\ell}\left(k_{\infty}\right)_{(0)} \stackrel{\alpha}{\longrightarrow} \mathrm{Cl}_{S}(k)_{\ell} \stackrel{\beta}{\longleftarrow} \mathrm{Cl}(k)_{\ell},
$$

где $\alpha$ индуцировано включением $k_{S, \ell} \subset M_{\infty}, M_{\infty}$ - поле, определенное в $\S 3$, а $\beta$ означает факторизацию по подгруппе $P_{S}(k)$. Таким образом, отображение $\beta$ всегда эпиморфно, в то время как Coker $\alpha \cong G\left(k_{s_{0}} / k\right)$, где $s_{0} \geqslant 0$ - максимальный индекс такой, что все точки $v \mid \ell$ вполне распадаются в $k_{s_{0}} / k$.

Чтобы интерпретировать группы $\operatorname{Ker} \alpha$ и $\operatorname{Ker} \beta$, мы положим $\mathcal{B}\left(k_{v}\right)=$ $\left(k_{v}^{*} / \mu\left(k_{v}\right)\right)[\ell]$ для любой точки $v \mid \ell$ и положим $\mathcal{B}(k)=\prod_{v \mid \ell} \mathcal{B}\left(k_{v}\right)$. Обозначим через $\Gamma_{v}$ подгрупту разложения $G\left(k_{\infty, v} / k_{v}\right)$. Тогда согласно локальной теории полей классов имеется естественный эпиморфизм $\lambda_{v}: \mathcal{B}\left(k_{v}\right) \rightarrow \Gamma_{v}$. С другой стороны, имеется эпиморфизм $\eta_{v}: \mathcal{B}\left(k_{v}\right) \rightarrow D_{v}$, где $D_{v} \cong \mathbf{Z}_{\ell}$, и для $x \in k_{v}^{*}, x=\pi_{v}^{n} u$, где $u \in U\left(k_{v}\right)$ и $\pi_{v}-$ локальньй параметр поля $k_{v}$, мы полагаем $\eta_{v}(x)=n$. Тогда, полагая $\mathcal{R}=\prod_{v \mid \ell} \Gamma_{v}$, мы получаем естественные $G$-гомоморфизмы

$$
\lambda=\prod_{v \mid \ell} \lambda_{v}: \mathcal{B}(k) \rightarrow \mathcal{R}, \quad \eta=\prod_{v \mid \ell} \eta_{v}: \mathcal{B}(k) \rightarrow D_{\ell}:=\prod_{v \mid \ell} D_{v}
$$


где $G$ действует на $\mathcal{R}$ и $D_{\ell}$ посредством действия на множество $S$ всех точек поля $k$, лежаших над $\ell$. Таким образом, $\mathcal{R} \cong D_{\ell} \cong \mathbf{Z}_{\ell}\left[G / G_{v}\right]$ как модули Галуа. Поскольку $\Gamma_{v}$ является подгруппой разложения точки $v$ в группе $\bar{\Gamma}=G\left(k_{\infty} / k\right)$, мы получаем естественное отображение $\mathcal{R} \rightarrow \bar{\Gamma}$, ядро которого совпадает с $\mathcal{R}_{0}$. Тогда согласно глобальной теории полей классов мы получаем

$$
\operatorname{Ker} \alpha=\mathcal{R}_{0} / \lambda\left(\bar{U}_{S}(k)[\ell]\right) .
$$

С другой стороны, мы имеем

$$
\operatorname{Ker} \beta=P_{S}(k) \cong D_{\ell} / \eta\left(\bar{U}_{S}(k)[\ell]\right) .
$$

Согласно локальной теории полей классов для $x \in \mathcal{B}\left(k_{n, v}\right)$ равенство $\lambda_{v}(x)=0$ вьполняется тогда и только тогда, когда $x \in \bigcap_{n=1}^{\infty} N_{k_{n} / k} \mathcal{B}\left(k_{n, v}\right)$. Таким образом, мы получаем $\operatorname{Ker} \lambda=H(k)$. Из определения $\eta$ следует, что Ker $\eta=\mathcal{A}(k)$. Поэтому мы имеем

$$
\bar{U}_{S}(k)[\ell] \cap \operatorname{Ker} \lambda=\widehat{U}_{S}(k), \quad \bar{U}_{S}(k)[\ell] \cap \operatorname{Ker} \eta=\bar{U}(k)[\ell] .
$$

Положим

$$
\begin{aligned}
& V:=\operatorname{Ker} \lambda \cap \operatorname{Ker} \eta=H(k) \cap \mathcal{A}(k), \\
& E:=\bar{U}_{S}(k)[\ell] \cap \operatorname{Ker} \lambda \cap \operatorname{Ker} \eta=\widehat{U}_{S}(k) \cap \operatorname{Ker} \eta=\bar{U}(k)[\ell] \cap \operatorname{Ker} \lambda .
\end{aligned}
$$

Тогда имеется пара точных последовательностей (в аддитивной записи)

$$
\begin{gathered}
0 \longrightarrow V / E \longrightarrow \mathcal{A}(k)_{0} / \bar{U}(k)[\ell] \longrightarrow \mathcal{A}(k)_{0} / V \bar{U}(k)[\ell] \longrightarrow 0 \\
0 \longrightarrow V / E \longrightarrow H(k) / \widehat{U}_{S}(k) \longrightarrow H(k) / V \widehat{U}_{S}(k) \longrightarrow 0
\end{gathered}
$$

Таким образом, мы получаем

$$
\begin{gathered}
\frac{\left[\mathcal{A}(k)_{0}: \bar{U}(k)[\ell]\right]}{\left[H(k): \widehat{U}_{S}(k)\right]}=\frac{\left[\mathcal{A}(k)_{0}: V \bar{U}(k)[\ell]\right]}{\left[H(k): V \widehat{U}_{S}(k)\right]}, \\
\frac{\left[\mathcal{A}(k)_{0, \varphi}: \bar{U}(k)[\ell]_{\varphi}\right]}{\left[H(k)_{\varphi}: \widehat{U}_{S}(k)_{\varphi}\right]}=\frac{\left[\mathcal{A}(k)_{0, \varphi}:(V \bar{U}(k)[\ell])_{\varphi}\right]}{\left[H(k)_{\varphi}:\left(V \widehat{U}_{S}(k)\right)_{\varphi}\right]}
\end{gathered}
$$

для любого $\varphi \in \Phi$.

Для $v \mid \ell$ пусть $\bar{\Gamma}_{v}^{\prime}-$ подгруппа инерции точки $v$ в $\bar{\Gamma}$. Тогда $\Gamma_{v}^{\prime} \subseteq \Gamma_{v}$ и для любой точки $v \mid \ell\left[\Gamma_{v}: \Gamma_{v}^{\prime}\right]=\ell^{u}$, где $u=s-s_{0}$. Согласно локальной теории полей классов мы имеем $\lambda(\mathcal{A}(k))=\prod_{v \mid \ell} \Gamma_{v}^{\prime}=\ell^{u} \mathcal{R}$. Обозначим через $\lambda^{\prime}: \mathcal{B}(k) \rightarrow$ $\mathcal{R} / \ell^{u} \mathcal{R}$ композицию $\lambda$ и естественного отображения $\mathcal{R} \rightarrow \mathcal{R} / \ell^{u} \mathcal{R}$. Тогда $\operatorname{Ker} \lambda^{\prime}=$ $\mathcal{A}(k) \cdot H(k)$. Заметим, что $\lambda$ отображает $\mathcal{A}(k)_{0}$ на $\ell^{u} \mathcal{R}_{0}$. Следовательно, (7.10) индуцирует точную последовательность

$$
0 \longrightarrow \mathcal{A}(k)_{0} / V \bar{U}(k)[\ell] \longrightarrow \operatorname{Ker} \alpha \longrightarrow\left(\mathcal{R}_{0} / \ell^{u} \mathcal{R}_{0}\right) / \lambda^{\prime}\left(\bar{U}_{S}(k)[\ell]\right) \longrightarrow 0 .
$$

Обозначим теперь через $\eta^{\prime}: \mathcal{B}(k) \rightarrow D_{\ell} / \ell^{u} D_{\ell}$ композицию $\eta$ и естественного отображения $D_{\ell} \rightarrow D_{\ell} / \ell^{u} D_{\ell}$. Поскольку все точки $v \mid \ell$ поля $k_{s_{0}}$ остаются инертными в $k_{s} / k_{s_{0}}$ и вполне разветвлены в $k_{\infty} / k_{s}$, мы получаем, что включение $x \in H(k)$ влечет $\eta^{\prime}(x)=0$. С другой стороны, если $x \in \mathcal{B}(k)$ и $\eta^{\prime}(x)=0$, то можно найти $y \in \mathcal{A}(k)$ такой, что $x y \in H(k)$. Следовательно, 


$$
\operatorname{Ker} \eta^{\prime}=\mathcal{A}(k) \cdot H(k)=\operatorname{Ker} \lambda^{\prime},
$$

и (7.11) индуцирует точную последовательность

$$
0 \longrightarrow H(k) / V \widehat{U}_{S}(k) \longrightarrow \operatorname{Ker} \beta \longrightarrow\left(D_{\ell} / \ell^{u} D_{\ell}\right) / \eta^{\prime}\left(\bar{U}_{S}(k)[\ell]\right) \longrightarrow 0 .
$$

Так как $\mathcal{R} \cong D_{\ell}$, мы получаем, что $\mathcal{R} / \ell^{u} \mathcal{R} \cong D_{\ell} / \ell^{u} D_{\ell}$. Легко проверить, что $\left(\mathcal{R} / \ell^{u} \mathcal{R}\right) /\left(\mathcal{R}_{0} / \ell^{u} \mathcal{R}_{0}\right) \cong \mathbf{Z} / \ell^{u} \mathbf{Z}$ (с тривиальным действием $\left.G\right)$. Согласно (7.15) мы имеем

$$
\lambda^{\prime}\left(\bar{U}_{S}(k)[\ell]\right) \cong \bar{U}_{S}(k)[\ell] /\left(\bar{U}_{S}(k)[\ell] \cap \mathcal{A}(k) \cdot H(k)\right) \cong \eta^{\prime}\left(\bar{U}_{S}(k)[\ell]\right) .
$$

Тогда из (7.14) и (7.16) мы получаем

$$
\begin{gathered}
\frac{\left[\mathcal{A}(k)_{0}: V \bar{U}(k)[\ell]\right]}{\left[H(k): V \widehat{U}_{S}(k)\right]}=\ell^{-u} \frac{|\operatorname{Ker} \alpha|}{|\operatorname{Ker} \beta|} \\
\frac{\left[\mathcal{A}(k)_{0, \varphi}:(V \bar{U}(k)[\ell])_{\varphi}\right]}{\left[H(k)_{\varphi}:\left(V \widehat{U}_{S}(k)\right)_{\varphi}\right]}=\ell^{-u_{\varphi}} \frac{\left|(\operatorname{Ker} \alpha)_{\varphi}\right|}{\left|(\operatorname{Ker} \beta)_{\varphi}\right|}
\end{gathered}
$$

где $u_{\varphi}=0$ при $\varphi \neq \varphi_{0}$ и $u_{\varphi}=u$ при $\varphi=\varphi_{0}$.

Сопоставляя формулы (7.12), (7.17), (7.9), мы получаем первую формулу теоремы. Чтобы получить последние две формулы теоремы, мы должны сопоставить формулы (7.13), (7.18) и (7.9). Теорема доказана.

Следуюший результат является аналогом теоремы 5.1.

Теорема 7.2. Для любого вещественного абелева поля $k$ и любого $\varphi \in \Phi$ миц имеем

$$
\begin{aligned}
\nu_{\ell}\left(\left|\mathrm{Cl}(k)_{\ell}\right|\right) & =\nu_{\ell}\left(\ell^{s+t}|Y(k)|^{-1}\left[\mathcal{A}(k)_{0}: \bar{U}(k)[\ell]\right]^{-1} \prod_{\substack{\chi \in \widehat{G} \\
\chi \neq 1}} \frac{1}{2} L_{\ell}(1, \chi)\right), \\
\nu_{\ell}\left(\left|\mathrm{Cl}(k)_{\ell, \varphi}\right|\right) & =\nu_{\ell}\left(\ell^{s \varphi+t_{\varphi}}\left|Y(k)_{\varphi}\right|^{-1}\left[\mathcal{A}(k)_{0, \varphi}: \bar{U}(k)[\ell]_{\varphi}\right]^{-1} \prod_{\substack{\chi \in \widehat{G} \\
\chi \mid \varphi, \chi \neq 1}} \frac{1}{2} L_{\ell}(1, \chi)\right),
\end{aligned}
$$

где $t, t_{\varphi}$ были определены в теореме $5.1, s$ имеет тот же смысл, что и в предложсении 7.4 и $s_{\varphi}=0$ для $\varphi \neq \varphi_{0}, s_{\varphi_{0}}=s$.

ДокАЗАТЕльСтво. Наша теорема немедленно следует из теорем 5.1 и 7.1.

Положим $\widetilde{U}(k)=\overline{\mathcal{O}} \cdot \log (\bar{U}(k)[\ell])$, где $\log$ - отображение, определенное в (7.2). Тогда индекс $\left(\widetilde{\mathcal{A}}(k)_{0}: \widetilde{U}(k)\right)$ существует и

$$
\left(\widetilde{\mathcal{A}}(k)_{0}: \widetilde{U}(k)\right)=\left(\mathcal{A}(k)_{0}: \bar{U}(k)[\ell]\right), \quad\left(\widetilde{\mathcal{A}}(k)_{0, \varphi}: \widetilde{U}(k)_{\varphi}\right)=\left(\mathcal{A}(k)_{0, \varphi}: \bar{U}(k)[\ell]_{\varphi}\right)
$$


О ФОРМУЛАХ ДЛЯ ЧИСЛА КЛАССОВ ВЕШЕСТВЕННЫХ АБЕЛЕВЫХ ПОЛЕЙ 89

ТЕорема 7.3. Для любого вещественного абелева поля $k$ и любого $\varphi \in \Phi$ мы имеем

$$
\begin{aligned}
\nu_{\ell}\left(\left|\mathrm{Cl}(k)_{\ell}\right|\right) & =\nu_{\ell}\left(\left(\mathbf{R}_{k, 0}: \widetilde{U}(k)\right)^{-1} \prod_{\substack{\chi \in \widehat{G} \\
\chi \neq 1}} \frac{1}{2} a(\chi)\right), \\
\nu_{\ell}\left(\left|\mathrm{Cl}(k)_{\ell, \varphi}\right|\right) & =\nu_{\ell}\left(\left(\mathbf{R}_{k, 0, \varphi}: \widetilde{U}(k)_{\varphi}\right)^{-1} \prod_{\substack{\chi \in \widehat{G} \\
\chi \mid \varphi, \chi \neq 1}} \frac{1}{2} a(\chi)\right),
\end{aligned}
$$

где $\partial л я ~ \chi \neq 1$

$$
a(\chi)=\sum_{\substack{a \bmod f_{\chi} \\\left(a, f_{\chi}\right)=1}} \bar{\chi}(a) \log \left(1-\varepsilon_{\chi}^{a}\right),
$$

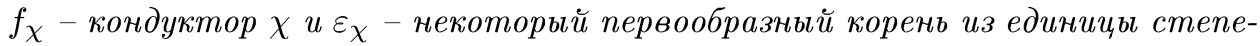
ни $f_{\chi}$ (заметим, что $\nu_{\ell}(a(\chi))$ не зависит от въбора $\left.\varepsilon_{\chi}\right)$.

ДокАЗАТЕЛЬСТво. Мы имеем

$$
\left(\mathbf{R}_{k, 0}: \widetilde{U}(k)\right)=\left(\mathbf{R}_{k, 0}: \widetilde{\mathcal{O}}_{k, 0}\right)\left(\widetilde{\mathcal{O}}_{k, 0}: \widetilde{\mathcal{A}}_{k, 0}\right)\left(\widetilde{\mathcal{A}}_{k, 0}: \widetilde{U}(k)\right)
$$

и

$$
\left(\mathbf{R}_{k, 0, \varphi}: \widetilde{U}(k)_{\varphi}\right)=\left(\mathbf{R}_{k, 0, \varphi}: \widetilde{\mathcal{O}}_{k, 0, \varphi}\right)\left(\widetilde{\mathcal{O}}_{k, 0, \varphi}: \widetilde{\mathcal{A}}_{k, 0, \varphi}\right)\left(\widetilde{\mathcal{A}}_{k, 0, \varphi}: \widetilde{U}(k)_{\varphi}\right)
$$

для любого $\varphi \in \Phi$.

Из (7.3) следует, что

$$
\begin{aligned}
{\left[\mathcal{A}(k)_{0}: \bar{U}(k)[\ell]\right] } & =\left(\widetilde{\mathcal{A}}_{k, 0}: \widetilde{U}(k)\right), \\
{\left[\mathcal{A}(k)_{0, \varphi}: \bar{U}(k)[\ell]_{\varphi}\right] } & =\left(\widetilde{\mathcal{A}}_{k, 0, \varphi}: \widetilde{U}(k)_{\varphi}\right)
\end{aligned}
$$

для любого $\varphi \in \Phi$.

Тогда из теоремы 7.2 мы получаем

$$
\begin{aligned}
& \nu_{\ell}\left(\left|\mathrm{Cl}(k)_{\ell}\right|\right)=\nu_{\ell}\left(\ell^{s+t}|Y(k)|^{-1}\left(\mathbf{R}_{k, 0}: \widetilde{U}(k)\right)^{-1}\right. \\
& \left.\times\left(\mathbf{R}_{k, 0}: \widetilde{\mathcal{O}}_{k, 0}\right)\left(\widetilde{\mathcal{O}}_{k, 0}: \widetilde{\mathcal{A}}_{k, 0}\right) \prod_{\substack{\chi \in \widehat{G} \\
\chi \neq 1}} \frac{1}{2} L_{\ell}(1, \chi)\right) \\
& \nu_{\ell}\left(\left|\mathrm{Cl}(k)_{\ell, \varphi}\right|\right)=\nu_{\ell}\left(\ell^{s}+t_{\varphi}\left|Y(k)_{\varphi}\right|^{-1}\right)+\nu_{\ell}\left(\left(\mathbf{R}_{k, 0, \varphi}: \widetilde{U}(k)_{\varphi}\right)^{-1}\right. \\
& \times\left(\mathbf{R}_{k, 0, \varphi}: \widetilde{\mathcal{O}}_{k, 0, \varphi}\right)\left(\widetilde{\mathcal{O}}_{k, 0, \varphi}: \widetilde{\mathcal{A}}_{k, 0, \varphi} \prod_{\substack{\chi \in \widehat{G} \\
\chi \mid \varphi, \chi \neq 1}} \frac{1}{2} L_{\ell}(1, \chi)\right) .
\end{aligned}
$$

Из теоремы 5.1 и предложения 7.4 следует, что

$$
s+t= \begin{cases}m-2 & \text { для } \ell=2 \text { и } k \text { типа }(\mathrm{C}), \\ m-1 & \text { во всех остальных случаях }\end{cases}
$$


и

$$
s_{\varphi}+t_{\varphi}=0 \text { при } \varphi \neq \varphi_{0}, \quad s_{\varphi_{0}}+t_{\varphi_{0}}=s+t .
$$

Из предложений 7.1-7.3 и теоремы 6.1 следует, что

$$
\begin{gathered}
\nu_{\ell}\left(\left(\mathbf{R}_{k, 0}: \widetilde{\mathcal{O}}_{k, 0}\right)\left(\widetilde{\mathcal{O}}_{k, 0}: \widetilde{\mathcal{A}}_{k, 0}\right)\right)=\nu_{\ell}\left(|P(k)| \cdot|Y(k)| \cdot \ell^{a-c} \prod_{\substack{\chi \in \widehat{G} \\
\chi \neq 1}} g_{\bar{\chi}}\right), \\
\nu_{\ell}\left(\left(\mathbf{R}_{k, 0, \varphi}: \widetilde{\mathcal{O}}_{k, 0, \varphi}\right)\left(\widetilde{\mathcal{O}}_{k, 0, \varphi}: \widetilde{\mathcal{A}}_{k, 0, \varphi}\right)\right) \\
=\nu_{\ell}\left(\left|P(k)_{\varphi}\right| \cdot\left|Y(k)_{\varphi}\right| \cdot \ell^{a_{\varphi}-c_{\varphi}} \prod_{\substack{\chi \in \widehat{G} \\
\chi \mid \varphi, \chi \neq 1}} g_{\bar{\chi}}\right),
\end{gathered}
$$

где $a$ (соответственно $c$ ) определено в предложении 7.2 (соответственно в предложении 7.3), $a_{\varphi}=c_{\varphi}=0$ для $\varphi \neq \varphi_{0}$, и $a_{\varphi_{0}}=a, c_{\varphi_{0}}=c$.

Если $m=0$, то

$$
a-c= \begin{cases}-1 & \text { при } \ell \neq 2 \\ -2 & \text { при } \ell=2 .\end{cases}
$$

Если $m>0$, то

$$
a-c= \begin{cases}-m+1 & \text { для } \ell=2 \text { и } k \text { типа }(\mathrm{C}), \\ -m & \text { во всех остальньх случаях. }\end{cases}
$$

Следовательно, в любом случае мы имеем $s+t+a-c=-1$. Тогда из (7.20) и (7.21) следует, что

$$
\begin{aligned}
\nu_{\ell}\left(\left|\mathrm{Cl}(k)_{\ell}\right|\right)= & \nu_{\ell}\left(\left(\mathbf{R}_{k, 0}: \widetilde{U}(k)\right)^{-1} \cdot \ell^{-1}|P(k)| \prod_{\substack{\chi \in \widehat{G} \\
\chi \neq 1}} \frac{1}{2} g_{\bar{\chi}} L_{\ell}(1, \chi)\right), \\
\nu_{\ell}\left(\left|\mathrm{Cl}(k)_{\ell, \varphi}\right|\right)= & \nu_{\ell}\left(\left(\mathbf{R}_{k, 0, \varphi}: \widetilde{U}(k)_{\varphi}\right)^{-1}\right. \\
& \left.\times \ell^{b}\left|P(k)_{\varphi}\right| \prod_{\substack{\chi \in \widehat{G} \\
\chi \mid \varphi, \chi \neq 1}} \frac{1}{2} g_{\bar{\chi}} L_{\ell}(1, \chi)\right),
\end{aligned}
$$

где $b=-1$ при $\varphi=\varphi_{0}, b=0$ при $\varphi \neq \varphi_{0}$.

Хорошо известно (см., например, [12]), что для $\chi \neq 1$

$$
L_{\ell}(1, \chi)=-\left(1-\frac{\chi(\ell)}{\ell}\right) \frac{g_{\chi}}{f_{\chi}} a(\chi) \text {. }
$$

Поскольку $g_{\chi} g_{\bar{\chi}}=f_{\chi} \cdot \chi(-1)$, мы имеем

$$
g_{\bar{\chi}} L_{\ell}(1, \chi)= \pm\left(1-\frac{\chi(\ell)}{\ell}\right) a(\chi)
$$


Заметим, что

$$
\nu_{\ell}\left(1-\frac{\chi(\ell)}{\ell}\right)=\left\{\begin{array}{lll}
0 & \text { при } & \chi(\ell)=0 \\
-1 & \text { при } & \chi(\ell) \neq 0
\end{array}\right.
$$

Пусть $k^{\prime}$ - максимальное подполе поля $k$ такое, что $\ell$ не разветвлено в $k^{\prime} / \mathbf{Q}$. Тогда для $\chi \in \widehat{G}$ равенство $\chi(\ell) \neq 0$ выполняется тогда и только тогда, когда $\left.\chi\right|_{G\left(k / k^{\prime}\right)}=1$, другими словами, когда $\chi$ является характером группы $G\left(k^{\prime} / \mathbf{Q}\right)$. Учитывая, что $P(k) \cong \mathbf{Z} / \ell \mathbf{Z}\left[G\left(k^{\prime} / \mathbf{Q}\right)\right]$ как модуль Галуа, мы получаем

$$
\begin{gathered}
\nu_{\ell}\left(\prod_{\substack{\chi \in \widehat{G} \\
\chi \neq 1}}\left(1-\frac{\chi(\ell)}{\ell}\right)\right)=\nu_{\ell}(\ell|P(k)|), \\
\nu_{\ell}\left(\prod_{\substack{\chi \in \widehat{G} \\
\chi \mid \varphi, \chi \neq 1}}\left(1-\frac{\chi(\ell)}{\ell}\right)\right)=\nu_{\ell}\left(\ell^{-b}\left|P(k)_{\varphi}\right|\right),
\end{gathered}
$$

где $b=-1$ при $\varphi=\varphi_{0}$ и $b=0$ при $\varphi \neq \varphi_{0}$. Сопоставляя формулы (7.22), (7.24) и (7.25), мы получаем первую формулу теоремы. Чтобы получить вторую формулу, следует сопоставить формулы (7.23), (7.24) и (7.26). Теорема доказана.

ЗАмЕчаниЕ 1 . Хорошо известно, что для любого $\chi \in \widehat{G}$ такого, что $\chi \neq 1$, мы имеем $a(\chi) \neq 0$.

ЗАмечание 2. Теорему 7.3 можно рассматривать как $\ell$-адическую аналитическую формулу для числа классов, дающую порядок групшы $\mathrm{Cl}(k)_{\ell, \varphi}$. Индекс $\left(\mathbf{R}_{k, 0, \varphi}: \widetilde{U}(k)_{\varphi}\right)$ может рассматриваться как $\varphi$-компонента $\ell$-адического регулятора поля $k$.

Для вещественного абелева поля $k$ с группой Галуа $G=G(k / \mathbf{Q})$ положим $\mathcal{R}_{k}=\mathbf{Z}_{\ell}[G]$. Пусть $\mathbf{R}_{k}$ имеет тот же смысл, что и в теореме 7.3. Предположим, что нам задан некоторый $\mathcal{R}_{k}$-подмодуль $\mathcal{U}_{k} \subset \mathbf{Q}_{\ell}[G]$ такой, что определен индекс $\left(\mathcal{R}_{k, 0}: \mathcal{U}_{k, 0}\right)$. Положим $\mathbf{U}_{k}=\overline{\mathcal{O}} \cdot \mathcal{U}_{k} \subset \mathbf{A}_{k}$. Пусть задан некоторый $\mathcal{R}_{k}$-подмодуль $\mathcal{T}_{k}$ модуля $\bar{U}(k)[\ell] \otimes \mathbf{z}_{\ell} \mathbf{Q}_{\ell}$ такой, что группа $\bar{U}(k)[\ell] \cap \mathcal{T}_{k}$ имеет конечньй индекс и в $\bar{U}(k)[\ell]$ и в $\mathcal{T}_{k}$. (Отметим, что $\mathcal{T}_{k}=\mathcal{T}_{k, 0}$.) Пусть задана подгруппа $C_{k} \subseteq \bar{U}(k)[\ell] \cap \mathcal{T}_{k}$ такая, что $C_{k}$ имеет конечный индекс и в $\bar{U}(k)[\ell]$ и в $\mathcal{T}_{k}$. Положим $\mathbf{T}_{k}=\overline{\mathcal{O}} \cdot \log \left(\mathcal{T}_{k}\right)$ и $\mathbf{C}_{k}=\overline{\mathcal{O}} \cdot \log C_{k}$, где $\log$ совпадает с отображением (7.2).

Теорема 7.4. Пусть $\mathcal{R}_{k}, \mathbf{R}_{k}, \mathcal{U}_{k}, \mathbf{U}_{k}, \mathcal{T}_{k}, \mathbf{T}_{k} u C_{k}, \mathbf{C}_{k}$ определены, как указано выше. Предположим, что $\mathbf{U}_{k, 0}$ и $\mathbf{T}_{k}$ изоморфны как $\mathbf{R}_{k}$-модули и $e_{\chi} \mathbf{T}_{k}=\frac{1}{2} a(\chi) e_{\chi} \mathbf{U}_{k}$ для любого $\chi \in \widehat{G} \quad \chi \neq 1$. Тогда

$$
\left|\mathrm{Cl}(k)_{\ell}\right|=\left(\mathcal{R}_{k, 0}: \mathcal{U}_{k, 0}\right)^{-1}\left[\mathcal{T}_{k}: C_{k}\right]^{-1}\left[\bar{U}(k)[\ell]: C_{k}\right]
$$

$u$

$$
\left|\mathrm{Cl}(k)_{\ell, \varphi}\right|=\left(\mathcal{R}_{k, 0, \varphi}: \mathcal{U}_{k, 0, \varphi}\right)^{-1}\left[\mathcal{T}_{k, \varphi}: C_{k, \varphi}\right]^{-1}\left[\bar{U}(k)[\ell]_{\varphi}: C_{k, \varphi}\right]
$$

для любого $\varphi \in \Phi$. 
ДоКАЗАТЕЛЬСТво. Согласно лемме 6.4 мы имеем

$$
\nu_{\ell}\left(\left(\mathbf{U}_{k, 0}: \mathbf{T}_{k}\right)\right)=\nu_{\ell}\left(\prod_{\substack{\chi \in \widehat{G} \\ \chi \neq 1}} \frac{1}{2} a(\chi)\right)
$$

и

$$
\nu_{\ell}\left(\left(\mathbf{U}_{k, 0, \varphi}: \mathbf{T}_{k, \varphi}\right)\right)=\nu_{\ell}\left(\prod_{\substack{\chi \in \widehat{G} \\ \chi \mid \varphi, \chi \neq 1}} \frac{1}{2} a(\chi)\right)
$$

для любого $\varphi \in \Phi$. Тогда по теореме 7.3 мы получаем

$$
\begin{array}{r}
\nu_{\ell}\left(\left|\mathrm{Cl}(k)_{\ell}\right|\right)=\nu_{\ell}\left(\left(\mathbf{R}_{k, 0}: \widetilde{U}(k)\right)^{-1} \prod_{\substack{\chi \in \widehat{G} \\
\chi \neq 1}} \frac{1}{2} a(\chi)\right) \\
=\left(\mathbf{R}_{k, 0}: \mathbf{U}_{k, 0}\right)^{-1}\left(\widetilde{U}(k): \mathbf{R}_{k, 0}\right)\left(\mathbf{R}_{k, 0}: \mathbf{U}_{k, 0}\right)\left(\mathbf{U}_{k, 0}: \mathbf{T}_{k}\right)\left(\mathbf{T}_{k}: \mathbf{C}_{k}\right)\left(\mathbf{T}_{k}: \mathbf{C}_{k}\right)^{-1} \\
=\left(\mathbf{R}_{k, 0}: \mathbf{U}_{k, 0}\right)^{-1}\left(\mathbf{T}_{k}: \mathbf{C}_{k}\right)^{-1}\left(\widetilde{U}(k): \mathbf{C}_{k}\right) .
\end{array}
$$

Согласно (6.4) мы имеем

$$
\begin{aligned}
\left(\mathbf{R}_{k, 0}: \mathbf{U}_{k, 0}\right) & =\left(\mathcal{R}_{k, 0}: \mathcal{U}_{k, 0}\right) \\
\left(\mathbf{T}_{k}: \mathbf{C}_{k}\right) & =\left(\mathcal{T}_{k}: C_{k}\right)=\left[\mathcal{T}_{k}: C_{k}\right] \\
\left(\widetilde{U}(k): \mathbf{C}_{k}\right) & =\left(\bar{U}(k)[\ell]: C_{k}\right)=\left[\bar{U}(k)[\ell]: C_{k}\right]
\end{aligned}
$$

Этим доказана первая формула теоремы. Вторая формула может быть доказана аналогичными рассуждениями.

\section{§8. Доказательство теоремы 1}

В этом параграфе мы определим модули $\mathcal{U}_{k}$ и $\mathcal{T}_{k}$, удовлетворяющие условиям теоремы 7.4 для случая, когда $k=K^{+}=\mathbf{Q}\left(\varepsilon_{m}+\varepsilon_{m}^{-1}\right)$ - максимальное вещественное подполе кругового поля $K=\mathbf{Q}\left(\varepsilon_{m}\right)$. Наши результаты опираются на результаты работы [15], поэтому мы будем использовать здесь ряд обозначений из [15].

Пусть $\mathcal{G} \cong(\mathbf{Z} / m \mathbf{Z})^{*}$ - группа Галуа расширения $K / \mathbf{Q}$. Если $m=\prod_{i} p_{i}^{s_{i}}$-разложение $m$ на простые множители, то $\mathcal{G}$ является прямым произведением подгрупп инерции $T_{p_{i}} \cong\left(\mathbf{Z} / p_{i}^{s_{i}} \mathbf{Z}\right)^{*}$ :

$$
\mathcal{G}=\prod_{p \mid m} T_{p}
$$

Для $p \mid m$ положим $e_{p}=\left|T_{p}\right|^{-1} \sum_{\sigma \in T_{p}} \sigma$. Как и в [15], мы положим $\bar{\sigma}_{p}=\lambda_{p}^{-1} e_{p}$, где $\lambda_{p} \in \mathcal{G}$ и $\lambda_{p} \bmod T_{p}$ совпадает с автоморфизмом Фробениуса расширения $K^{T_{p}} / \mathbf{Q}$, соответствуюшим $p$. Для $f \mid m$ пусть $H_{f}$ - ядро естественного эпиморфизма $(\mathbf{Z} / m \mathbf{Z})^{*} \rightarrow(\mathbf{Z} / f \mathbf{Z})^{*}$. Положим $\mathcal{R}:=\mathbf{Z}_{\ell}[\mathcal{G}]$.

Мы определим $\mathcal{R}$-модуль $\mathcal{U} \subset \mathbf{Q}_{\ell}[\mathcal{G}]$ как $\ell$-пополнение $\mathbf{Z}[\mathcal{G}]$-модуля $\mathcal{U}$, определенного в $\left[15\right.$, раздел 2], т.е. $\mathcal{U}$ совпадает с $\mathbf{Z}_{\ell}[\mathcal{G}]$-модулем, порожденным в $\mathbf{Q}_{\ell}[\mathcal{G}]$ элементами

$$
\alpha_{f}=s\left(H_{f}\right) \prod_{p \mid f}\left(1-\bar{\sigma}_{p}\right), \quad 1 \leqslant f \leqslant m, \quad f \mid m,
$$


где произведение берется по всем простым $p$, делящим $f$. Следуюшее утверждение является непосредственным следствием предложения 5.1 из [15].

ПРЕДЛОЖЕНИЕ 8.1. Для простого числа $p \mid$ m определим $\mathcal{U}_{p} \kappa a \kappa \mathcal{R}$-модуль, порожденныи в $\mathbf{Q}_{\ell}[\mathcal{G}]$ әлементами $s\left(T_{p}\right)=\sum_{\sigma \in T_{p}} \sigma u 1-\bar{\sigma}_{p}$ :

$$
\mathcal{U}_{p}=\mathcal{R} s\left(T_{p}\right)+\mathcal{R}\left(1-\bar{\sigma}_{p}\right) .
$$

Тогда

$$
\mathcal{U}=\prod_{p \mid m} \mathcal{U}_{p}
$$

где произведение берется по всем простым $p \mid m, a$ П означает произведение в групповой алгебре $\mathbf{Q}_{\ell}[\mathcal{G}]$.

Как и в [15], мы положим $\bar{m}=\prod_{p \mid m} p$, и для любого $r \mid \bar{m}$ положим

$$
\mathcal{U}_{r}=\prod_{p \mid r} \mathcal{U}_{p}, \quad T_{r}=\prod_{p \mid r} T_{p}
$$

По определению мы имеем $\mathcal{U}_{1}=\mathcal{R}, T_{1}=\{1\}$ и $\mathcal{U}_{\bar{m}}=\mathcal{U}, T_{\bar{m}}=\mathcal{G}$. Заметим, что любая группа $\mathcal{U}_{p}$ является полной $\mathbf{Z}_{\ell}$-решеткой в $\mathbf{Q}_{\ell}[\mathcal{G}]$.

Если $r|\bar{m}, p| \bar{m}$ и $(p, r)=1$, то имеется пара точных последовательностей $\mathcal{R}$-модулей

$$
\begin{aligned}
& 0 \longrightarrow \mathcal{U}_{r}^{T_{p}} \longrightarrow \mathcal{U}_{r} \longrightarrow Y \longrightarrow 0, \\
& 0 \longrightarrow \mathcal{U}_{r p}^{T_{p}} \longrightarrow \mathcal{U}_{r p} \longrightarrow Y \longrightarrow 0
\end{aligned}
$$

$($ см. $[15,(5.3)$ и $(5.4)])$. Здесь $Y=\left(1-e_{p}\right) \mathcal{U}_{r}=\left(1-e_{p}\right) \mathcal{U}_{r p}$, а эпиморфизмы в $(8.2)$ и (8.3) совпадают с отображениями, индуцированными умножением на $1-e_{p}$.

Следуюшие две леммы являются точными аналогами для случая $\mathbf{Q}_{\ell}[\mathcal{G}]$-модулей лемм 5.1 и 5.2 из [15] и могут быть доказаны тем же способом.

Лемма 8.1. Пусть $H$ - подгруппа группь $\mathcal{G}$ такая, что $H \cap T_{p}=\{1\}$. Пусть $A$ - произвольныи $T_{p}$-подмодуль в $\mathbf{Q}_{\ell}[\mathcal{G}]$ такой, что А свободен над $\mathbf{Z}_{\ell}\left[H T_{p}\right]$. Тогда и $A^{T_{p}}$ u $\left(1-e_{p}\right) A$ свободнын над $\mathbf{Z}_{\ell}[H]$.

Лемма 8.2. Пусть $A$ - некоторый $\mathcal{R}$-подмодуль в $\mathbf{Q}_{\ell}[\mathcal{G}]$. Тогда

$$
\left(A \mathcal{U}_{p}\right)^{T_{p}}=s\left(T_{p}\right) A+\left(1-\lambda_{p}^{-1}\right) A^{T_{p}} .
$$

Следовательно, если А свободен над $T_{p}$, то

$$
\left(A \mathcal{U}_{p}\right)^{T_{p}}=A^{T_{p}}=s\left(T_{p}\right) A .
$$

Начиная с этого места, мы зафиксируем некоторую циклическую подгруппу $H \subset \mathcal{G}$ такую, что $H \cap T_{r}=\{1\}$ для любого $r \mid \bar{m}, r \neq \bar{m}$. Для целей настоящего параграфа достаточно случая, когда $H=J=\{1, j\}$, где $j$ - автоморфизм комплексного сопряжения. В следуюшем параграфе мы встретимся с некоторыми другими типами $H$.

ПрЕДЛОЖЕНИЕ 8.2. Пусть $r$ и $r^{\prime}-$ взаимно простые делители $\bar{m}$. Тог-

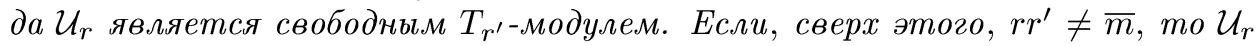

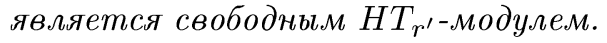


Чтобы доказать это предложение, следует повторить доказательство предложения 5.2 из [15], заменяя $U_{r}$ на $\mathcal{U}_{r}$ и $J$ на $H$. Отметим, что единственное свойство $J$, используемое в этом доказательстве, состоит в том, что $J \cap T_{r}=\{1\}$ для любого $r \neq \bar{m}$.

В качестве следствия мы получаем, что для $(p, r)=1$

$$
\mathcal{U}_{r}^{T_{p}}=\mathcal{U}_{r p}^{T_{p}} .
$$

В самом деле, $\mathcal{U}_{r} T_{p}$-свободен, следовательно, $\mathcal{U}_{r}^{T_{p}}=s\left(T_{p}\right) \mathcal{U}_{r}$ и $(8.4)$ вытекает из леммы 8.2.

Теперь мы перейдем к вычислению некоторых групп когомологий, связаных с $\mathcal{U}$ и $\mathcal{U}_{r}$. Пусть $A$ - некоторый $\mathcal{R}$-модуль и $F$ - некоторая подгруппа в $\mathcal{G}$. Тогда групшы когомологий Тэйта $H^{q}(F, A)$ являются $\mathcal{G} / F$-модулями и, следовательно, естественным образом могут рассматриваться как $\mathcal{G}$-модули. Если $E \supseteq F$ - некоторые подгруппы $\mathcal{G}$, то Res: $H^{q}(E, A) \rightarrow H^{q}(F, A)$ и Inf: $H^{q}\left(E / F, A^{F}\right) \rightarrow H^{q}(E, A)$ являются $\mathcal{G}$-отображениями при любом $q>0$.

Следуюшее предложение является точным аналогом предложения 5.3 из [15] и может быть доказано теми же рассуждениями (с заменой $U_{r}$ на $\mathcal{U}_{r}$ и $J$ на $\left.H\right)$.

ПРЕДЛОЖЕНИЕ 8.3. Пусть $r u r^{\prime}$ взаимно просты. Предположим, что ни $r$, ни $r^{\prime}$ не равны $\bar{m}$. Тогда для всех $q>0$ мы имеем

$$
H^{q}\left(T_{r^{\prime}}, \mathcal{U}_{r}^{H}\right) \cong H^{q}\left(H T_{r^{\prime}}, \mathcal{U}_{r}\right) \cong H^{q}\left(H, \mathcal{U}_{r}^{T_{r^{\prime}}}\right) .
$$

Эти отображения являются изоморфизмами $\mathcal{G}$-модулей. Более того, эти группь нулевые, за исключением случая, когда $\mathrm{rr}^{\prime}=\bar{m}$.

Как и в $\left[15\right.$, раздел 5], для любого $q>0$ и любого $r \mid m$ мы полагаем $r^{\prime}=\bar{m} / r$ и $A_{r}^{q}=H^{q}\left(H, \mathcal{U}_{r}^{T_{r^{\prime}}}\right)$.

ЛЕмма 8.3. Для любого $q>0 u r \mid m$ группа $\mathcal{G}$ действует тривиально на $A_{r}^{q}$.

Эта лемма может быть доказана теми же аргументами, что и лемма 5.3 из [15].

ЛЕмма 8.4. Предположим, что $p \not$. Тогда для любого челого $q>0$ существует точная последовательность

$$
0 \longrightarrow A_{r}^{q} \longrightarrow A_{r p}^{q} \longrightarrow A_{r}^{q+1} \longrightarrow 0
$$

Эта лемма может быть доказана теми же аргументами, что и лемма 5.4 из [15].

ЗАмечАнИЕ. Из доказательства, данного в [15], следует, что вложения $A_{r}^{q} \rightarrow$ $A_{r p}^{q}$ леммы 8.4 индуцированы естественными включениями $H$-модулей:

$$
\mathcal{U}_{r}^{H}=\mathcal{U}_{r p}^{H} \hookrightarrow \mathcal{U}_{r p}
$$

ПРЕДЛОЖЕНИЕ 8.4. Пусть $n$ - число простых делителей $r$. Если $n=0$, то для любого $q$

$$
A_{1}^{q}= \begin{cases}0 & \text { при нечетном } q, \\ \mathbf{Z}_{\ell} /|H| \mathbf{Z}_{\ell} & \text { при четном } q .\end{cases}
$$

Ecлu $n>0$, mo

$$
A_{r}^{q} \cong\left(\mathbf{Z}_{\ell} /|H| \mathbf{Z}_{\ell}\right)^{2^{n-1}}
$$

для любого $q>0$. 
ДокАЗАТЕЛЬСТво. Поскольку группа $H$ циклическая, достаточно рассмотреть случай $q>0$. Если $n=0$, то $r=1, \mathcal{U}_{1}=\mathcal{R}$ и

$$
A_{1}^{q}=H^{q}\left(H, \mathcal{R}^{\mathcal{G}}\right)=H^{q}\left(H, \mathbf{Z}_{\ell}\right),
$$

откуда следует первое утверждение предложения. Поскольку $|H| \cdot A_{r}^{q}=0$ для любых $q, r$ и $A_{r}^{q}$ являются $\ell$-группами, мы получаем, что все $A_{r}^{q}$ являются $\left(\mathbf{Z}_{\ell} /|H| \mathbf{Z}_{\ell}\right)$-модулями. Используя лемму 8.4, мы можем доказать по индукции, что все модули $A_{r}^{q}$ свободны над $\left(\mathbf{Z}_{\ell} /|H| \mathbf{Z}_{\ell}\right)$. Теперь второе утверждение предложения следует из леммы 8.4. Предложение доказано.

Положим $G=\mathcal{G} / H, G=G_{\ell} \times G_{0}$, где $G_{\ell}$ - силовская $\ell$-подгруппа группы $G$ и $\left(\left|G_{0}\right|, \ell\right)=1$. Пусть $\Phi$ - множество всех $\mathbf{Q}_{\ell}$-неприводимых характеров групшы $G_{0}$.

TЕорема 8.1. Пусть $H-$ ииклическая подгруппа группь $G$ такая, что $H \cap T_{r}=\{1\}$ для любого $r \mid \bar{m}, r \neq \bar{m}$. Пусть $g-$ число различных простых делителей $m$. Если $g=1$, то

$$
\begin{aligned}
\nu_{\ell}\left(\left(\mathcal{U}^{H}: \mathcal{R}^{H}\right)\right) & =0, \\
\nu_{\ell}\left(\left(\mathcal{R}^{H}: \operatorname{Sp}_{H} \mathcal{U}\right)\right) & =\nu_{\ell}(|H|) .
\end{aligned}
$$

Eсли $g \geqslant 2, m o$

$$
\nu_{\ell}\left(\left(\mathcal{U}^{H}: \mathcal{R}^{H}\right)\right)=\nu_{\ell}\left(\left(\mathcal{R}^{H}: \operatorname{Sp}_{H} \mathcal{U}\right)\right)=2^{g-2} \nu_{\ell}(|H|) .
$$

Пусть $\Phi$ определено, как и выше. Тогда для любого $\varphi \in \Phi$ ми имеем: если $g=1$, то

$$
\nu_{\ell}\left(\left(\mathcal{U}_{\varphi}^{H}: \mathcal{R}_{\varphi}^{H}\right)\right)=0, \quad \nu_{\ell}\left(\left(\mathcal{R}_{\varphi}^{H}: \operatorname{Sp}_{H} \mathcal{U}_{\varphi}\right)\right)=\left\{\begin{array}{lll}
0 & n p u & \varphi \neq \varphi_{0} \\
\nu_{\ell}(|H|) & n p u & \varphi=\varphi_{0}
\end{array}\right.
$$

если $g \geqslant 2, m o$

$$
\nu_{\ell}\left(\left(\mathcal{U}_{\varphi}^{H}: \mathcal{R}_{\varphi}^{H}\right)\right)=\nu_{\ell}\left(\left(\mathcal{R}_{\varphi}^{H}: \operatorname{Sp}_{H} \mathcal{U}_{\varphi}\right)\right)=\left\{\begin{array}{lll}
0 & n p u & \varphi \neq \varphi_{0} \\
2^{g-2} \nu_{\ell}(|H|) & n p u & \varphi=\varphi_{0}
\end{array}\right.
$$

ДоКАЗАТЕЛЬСТво. Пусть $p_{1}, \ldots, p_{g}$ - все простые делители $m$. Положим $r_{i}=$ $p_{1} \cdots p_{i}, i=1, \ldots, g$ и $r_{0}=1$. Мы имеем $\mathcal{U}_{r_{0}}=\mathcal{R}, \mathcal{U}_{r_{g}}=\mathcal{U}_{\bar{m}}=\mathcal{U}$. Следовательно,

$$
\left(\mathcal{U}^{H}: \mathcal{R}^{H}\right)=\prod_{i=1}^{g}\left(\mathcal{U}_{r_{i}}^{H}: \mathcal{U}_{r_{i-1}}^{H}\right)
$$

и

$$
\left(\mathcal{U}_{\varphi}^{H}: \mathcal{R}_{\varphi}^{H}\right)=\prod_{i=1}^{g}\left(\mathcal{U}_{r_{i}, \varphi}^{H}: \mathcal{U}_{r_{i-1}, \varphi}^{H}\right)
$$

для любого $\varphi \in \Phi$.

Для некоторого $i=1, \ldots, g$ положим $r_{i-1}=r, p_{i}=p, r_{i}=r p$. Чтобы вычислить индекс $\left(\mathcal{U}_{r}^{H}: \mathcal{U}_{r p}^{H}\right)$, заметим, что точные последовательности (8.2) и (8.3) 
вместе с равенством (8.4) приводят к следующей паре точных последовательностей $G$-модулей

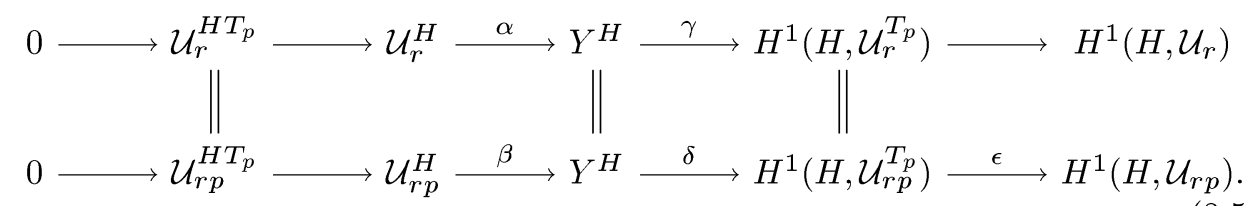

Так как $r \neq \bar{m}$, то из предложения 8.3 следует, что $H^{1}\left(H, \mathcal{U}_{r}\right)=0$, т.е. $\gamma$ является эпиморфизмом. С другой стороны, $\epsilon$ является мономорфизмом (см. замечание после леммы 8.4). Следовательно, $\delta=0$ и $\beta$ является эпиморфизмом. Таким образом, мы получаем

$$
\begin{aligned}
\left(\mathcal{U}_{r p}^{H}: \mathcal{U}_{r}^{H}\right) & =\left(\mathcal{U}_{r p}^{H T_{p}}: \mathcal{U}_{r}^{H T_{p}}\right)(\operatorname{Im} \beta: \operatorname{Im} \alpha) \\
& =(\operatorname{Im} \beta: \operatorname{Im} \alpha)=|\operatorname{Coker} \alpha| \cdot|\operatorname{Coker} \beta|^{-1}=|\operatorname{Coker} \alpha|=\left|H^{1}\left(H, \mathcal{U}_{r}^{T_{p}}\right)\right|
\end{aligned}
$$

Если $g=1$, то $r=1, \mathcal{U}_{r}^{T_{p}} \cong \mathbf{Z}_{\ell}$ и $H^{1}\left(H, \mathcal{U}_{r}^{T_{p}}\right)=0$. Следовательно, в этом случае $\left(\mathcal{U}^{H}: \mathcal{R}^{H}\right)=1$. Согласно предложению $8.4 \mathcal{U}^{H} / \operatorname{Sp}_{H} \mathcal{U} \cong\left(\mathbf{Z}_{\ell} /|H| \mathbf{Z}_{\ell}\right)$, следовательно, $\nu_{\ell}\left(\left(\mathcal{R}^{H}: \operatorname{Sp}_{H} \mathcal{U}\right)\right)=\nu_{\ell}(|H|)$.

Если $g>1$ и $r p \neq \bar{m}$ в (8.5), то в силу предложения $8.3 H^{1}\left(H, \mathcal{U}_{r}^{T_{p}}\right)=0$, поэтому согласно (8.6) мы имеем $\left(\mathcal{U}_{r p}^{H}: \mathcal{U}_{r}^{H}\right)=1$. Если $r p=\bar{m}$, то согласно (8.6) и предложению 8.4 мы имеем

$$
\nu_{\ell}\left(\left(\mathcal{U}_{r p}^{H}: \mathcal{U}_{r}^{H}\right)\right)=\nu_{\ell}\left(\left|H^{1}\left(H, \mathcal{U}_{r}^{T_{p}}\right)\right|\right)=2^{g-2} \nu_{\ell}(|H|) .
$$

Согласно предложению $8.4 \nu_{\ell}\left(\left(\mathcal{U}^{H}: \operatorname{Sp}_{H} \mathcal{U}\right)\right)=2^{g-1} \nu_{\ell}(|H|)$, следовательно, $\nu_{\ell}\left(\left(\mathcal{R}^{H}: \operatorname{Sp}_{H} \mathcal{U}\right)\right)=2^{g-2} \nu_{\ell}(|H|)$. Это доказывает первые два утверждения нашей теоремы.

Если $\varphi \in \Phi$, то, беря $\varphi$-компоненты всех групп, входящих в $(8.5)$, и рассуждая, как и прежде, мы получаем

$$
\left(\mathcal{U}_{r p, \varphi}^{H}: \mathcal{U}_{r, \varphi}^{H}\right)=\left|H^{1}\left(H, \mathcal{U}_{r}^{T_{p}}\right)_{\varphi}\right|
$$

Согласно лемме $8.3 \mathcal{G}$ действует тривиально на $H^{1}\left(H, \mathcal{U}_{r}^{T_{p}}\right)$, следовательно, мы имеем

$$
\left|H^{1}\left(H, \mathcal{U}_{r}^{T_{p}}\right)_{\varphi}\right|= \begin{cases}1 & \text { при } \varphi \neq \varphi_{0}, \\ \left|H^{1}\left(H, \mathcal{U}_{r}^{T_{p}}\right)\right| & \text { при } \varphi=\varphi_{0} .\end{cases}
$$

Это доказывает оставшуюся часть теоремы.

Заметим, что для любого $\mathcal{R}$-модуля $A \subset \mathbf{Q}_{\ell}[\mathcal{G}]$ мы имеем $\left(A_{0}\right)^{H}=\left(A^{H}\right)_{0}$ и $\left(\mathrm{Sp}_{H} A\right)_{0}=\operatorname{Sp}_{H}\left(A_{0}\right)$, поэтому мы можем просто писать $A_{0}^{H}$ и $\mathrm{Sp}_{H} A_{0}$.

ПРЕДЛОЖЕНИЕ 8.5. В предположсениях теоремы 8.1 мы имеем

$$
\begin{aligned}
\nu_{\ell}\left(\left(\mathcal{U}_{0}^{H}: \mathcal{R}_{0}^{H}\right)\right) & =\nu_{\ell}\left(\left(\mathcal{U}_{0, \varphi_{0}}^{H}: \mathcal{R}_{0, \varphi_{0}}^{H}\right)\right)=\nu_{\ell}\left(|\mathcal{G} / H| \cdot\left(\mathcal{U}^{H}: \mathcal{R}^{H}\right)\right), \\
\nu_{\ell}\left(\left(\mathcal{R}_{0}^{H}: \operatorname{Sp}_{H} \mathcal{U}_{0}\right)\right) & =\nu_{\ell}\left(\left(\mathcal{R}_{0, \varphi_{0}}^{H}: \operatorname{Sp}_{H} \mathcal{U}_{0, \varphi_{0}}\right)\right)=\nu_{\ell}\left(|\mathcal{G}|^{-1} \cdot\left(\mathcal{R}^{H}: \operatorname{Sp}_{H} \mathcal{U}\right)\right) .
\end{aligned}
$$


ДоКАЗАТЕЛЬСТВо. Положим $G=\mathcal{G} / H$. Тогда в силу (7.5) мы получаем

$$
\left(\mathcal{R}_{0}^{H}: \operatorname{Sp}_{H} \mathcal{U}_{0}\right)=\left(\mathcal{R}^{H}: \operatorname{Sp}_{H} \mathcal{U}\right)\left(\operatorname{Sp}_{G}\left(\operatorname{Sp}_{H} \mathcal{U}\right): \operatorname{Sp}_{G}\left(\mathcal{R}^{H}\right)\right)
$$

Заметим, что $\mathcal{R}$ свободен как $\mathcal{G}$-модуль, следовательно, $\mathcal{R}^{H}=\mathrm{Sp}_{H} \mathcal{R}$ и

$$
\left(\operatorname{Sp}_{G}\left(\operatorname{Sp}_{H} \mathcal{U}\right): \operatorname{Sp}_{G}\left(\mathcal{R}^{H}\right)\right)=\left(\operatorname{Sp}_{\mathcal{G}} \mathcal{U}: \operatorname{Sp}_{\mathcal{G}} \mathcal{R}\right)
$$

Очевидно, что $\operatorname{Sp}_{\mathcal{G}} \mathcal{R}=s(\mathcal{G}) \mathbf{Z}_{\ell}$. С другой стороны,

$$
\mathcal{U}=\mathcal{U}_{0}+s(\mathcal{G}) \mathbf{Z}_{\ell}
$$

(см. $[15,(4.11)])$. Следовательно, $\operatorname{Sp}_{\mathcal{G}} \mathcal{U}=|\mathcal{G}| s(\mathcal{G}) \mathbf{Z}_{\ell}$. Это доказывает последнюю формулу предложения. Чтобы доказать первую формулу, мы заметим, что в силу (8.6)

$$
\mathcal{U}^{H}=\left(\mathcal{U}_{0}+s(\mathcal{G}) \mathbf{Z}_{\ell}\right)^{H}=\mathcal{U}_{0}^{H}+s(\mathcal{G}) \mathbf{Z}_{\ell} .
$$

Следовательно, $\operatorname{Sp}_{G}\left(\mathcal{U}^{H}\right)=|G| s(\mathcal{G}) \mathbf{Z}_{\ell}$ и

$$
\left(\mathcal{U}_{0}^{H}: \mathcal{R}_{0}^{H}\right)=\left(\mathcal{U}^{H}: \mathcal{R}^{H}\right) \cdot\left(s(\mathcal{G}) \mathbf{Z}_{\ell}:|G| s(\mathcal{G}) \mathbf{Z}_{\ell}\right)=|G| \cdot\left(\mathcal{U}^{H}: \mathcal{R}^{H}\right) .
$$

Предложение доказано.

Если $K^{+}$- максимальное вешественное подполе поля $K=\mathbf{Q}\left(\varepsilon_{m}\right)$, то мы имеем $H=J=G\left(K / K^{+}\right)$и $G=G\left(K^{+} / \mathbf{Q}\right)$.

ОПРЕДЕЛЕНИЕ. Для поля $K^{+}$, определенного вьше, мы положим $\mathcal{U}_{K^{+}}=\operatorname{Sp}_{J}(\mathcal{U})$.

Следуюшее утверждение непосредственно следует из теоремы 8.1 и предложения 8.5.

ПРЕДЛОЖЕНИЕ 8.6. Пусть группа $\mathcal{U}_{K^{+}}$определена, как выще. Тогда

$$
\nu_{\ell}\left(\left(\mathcal{R}_{0}^{J}: \mathcal{U}_{K^{+}, 0}\right)\right)=\nu_{\ell}\left(|\mathcal{G}|^{-1} 2^{b_{1}}\right),
$$

ฉде

$$
b_{1}=\left\{\begin{array}{lll}
1 & n p u & g=1 \\
2^{g-2} & n p u & g \geqslant 2
\end{array}\right.
$$

Для любого $\varphi \in \Phi$ мы имеем

$$
\nu_{\ell}\left(\left(\mathcal{R}_{0, \varphi}^{J}: \mathcal{U}_{K^{+}, 0, \varphi}\right)\right)=\left\{\begin{array}{lll}
0 & \text { npu } & \varphi \neq \varphi_{0} \\
\nu_{\ell}\left(|\mathcal{G}|^{-1} 2^{b_{1}}\right) & \text { npu } & \varphi=\varphi_{0}
\end{array}\right.
$$

Теперь мы напомним некоторые результаты из [15], относяшиеся к круговым единицам и круговым числам. Пусть $P \subset K^{*}-$ группа круговых чисел, определенная в $[15$, раздел 4$]$, т.е. $P$ - подгруппа мультипликативной группы $K^{*}$ поля $K=\mathbf{Q}\left(\varepsilon_{m}\right)$, порожденная всеми элементами вида $1-\zeta$, где $\zeta \in K, \zeta^{m}=1, \zeta \neq 1$. Группа круговых единиц $C$ поля $K$ определяется равенством $C=P \cap E$, где $E$ группа единиц поля $K$. Заметим, что $\mu(K) \subset C$ (см. [15, раздел 1]), поэтому мы полагаем $\bar{P}=P / \mu(K), \bar{C}=C / \mu(K)$. В [15, раздел 4] было определено некоторое логарифмическое отображение $l$ группы $P$ такое, что $\operatorname{Ker} l=\mu(K)$. Поэтому модуль $T=l(P)$, определенный в [15], естественно изоморфен $\bar{P}$. Мы воспроизведем здесь некоторые результаты о модуле $T$, доказанные в [15]. 
Лемма 8.5 (см. [15, лемма 4.2]). Справедливо равенство $(C)=T_{0}$. Другими словами, $\bar{C}=\bar{P}_{0}$.

ЛЕмма 8.6 (см. [15, лемма 4.3]). Пусть $e_{1}=|\mathcal{G}|^{-1} s(\mathcal{G})$. Тогда $T_{0}=T \cap(1-$ $\left.e_{1}\right) T, T_{0}$ имеет конечный индекс в $\left(1-e_{1}\right) T$ и

$$
\left[\left(1-e_{1}\right) T: T_{0}\right]=2^{-g}|\mathcal{G}| .
$$

Другими словами, $\bar{C}=\bar{P} \cap\left(1-e_{1}\right) \bar{P} u$

$$
\left[\left(1-e_{1}\right) \bar{P}: \bar{C}\right]=2^{-g}|\mathcal{G}| \text {. }
$$

Учитьвая, что $\mathcal{G}$ действует тривиально на $\left(1-e_{1}\right) \bar{P} / \bar{C}$, мы можем переформулировать лемму 8.6 следуюшим образом:

Лемма 8.7. Пусть $\bar{C}[\ell]$ и $\bar{P}[\ell]$ означают $\ell$-пополнения $\bar{C}$ и $\bar{P}$ соответственно. Тогда $\bar{C}[\ell]$ имеет конечный индекс в $\left(1-e_{1}\right) \bar{P}[\ell]$ и для любого $\varphi \in \Phi$ мьи имеем

$$
\nu_{\ell}\left(\left[\left(1-e_{1}\right) \bar{P}[\ell]_{\varphi}: \bar{C}[\ell]_{\varphi}\right]\right)=\left\{\begin{array}{lll}
0 & \text { npu } & \varphi \neq \varphi_{0} \\
\nu_{\ell}\left(2^{-g}|\mathcal{G}|\right) & \text { npu } & \varphi=\varphi_{0}
\end{array}\right.
$$

ОПрЕДЕЛЕНИЕ. Пусть $K^{+}=\mathbf{Q}\left(\varepsilon_{m}+\varepsilon_{m}^{-1}\right)$ - максимальное вешественное подполе кругового поля $K=\mathbf{Q}\left(\varepsilon_{m}\right)$. Тогда мы полагаем $\mathcal{T}_{K^{+}}:=\left(1-e_{1}\right) \bar{P}[\ell]$.

Беря композицию диагонального вложения

$$
\bar{C}[\ell] \hookrightarrow \mathcal{A}\left(K^{+}\right)
$$

и отображения (7.2), мы получаем вложение

$$
\log : \bar{C}[\ell] \hookrightarrow \mathbf{A}_{K^{+}} .
$$

Продолжая отображение (8.9) по линейности на $\mathcal{T}_{K^{+}}$, мы получаем отображение

$$
\log : \mathcal{T}_{K^{+}} \hookrightarrow \mathbf{A}_{K^{+}} .
$$

Используя изоморфизм $\mathbf{A}_{K^{+}} \cong \overline{\mathbf{Q}}_{\ell}[G]$ из $\S 6$ и естественное вложение $\mathbf{Q}_{\ell}[G] \hookrightarrow$ $\overline{\mathbf{Q}}_{\ell}[G]$, мы получаем вложение $i: \mathcal{U}_{K^{+}} \hookrightarrow \mathbf{A}_{K^{+}}$.

ОПРедЕЛЕнИЕ. Мы полагаем $\mathbf{T}_{K^{+}}=\overline{\mathcal{O}} \cdot \log \left(\mathcal{T}_{K^{+}}\right)$и $\mathbf{U}_{K^{+}}=\overline{\mathcal{O}} \cdot i\left(\mathcal{U}_{K^{+}}\right)$.

Для $a \in \mathbf{Z}, a \neq \equiv(\bmod m)$, положим

$$
\eta(a):=l\left(1-\varepsilon_{m}^{a}\right):=\sum_{\substack{t \bmod m \\(t, m)=1}}-\frac{1}{2} \log \left(1-\varepsilon_{m}^{a t}\right) \sigma_{t}^{-1} \in \overline{\mathbf{Q}}_{\ell}[\mathcal{G}]=\mathbf{A}_{K} .
$$

Как и в $\left[15\right.$, раздел 2], через $V$ мы обозначим $\overline{\mathcal{O}}$-подмодуль в $\mathbf{A}_{K}$, порожденньй всеми элементами $\eta(a), a \in \mathbf{Z}, a \neq \equiv(\bmod m)$. Тогда $V$ является $\overline{\mathcal{O}}[\mathcal{G}]$-модулем и элементы $\eta(d), d \mid m$, порождают $V$ как $\mathbf{R}_{K}$-модуль.

Для $r=a / m, a \in \mathbf{Z}, a \neq \equiv(\bmod m)$, мы полагаем

$$
u(r)=-\frac{1}{2} \log \left(1-\varepsilon_{m}^{a}\right) \in \overline{\mathbf{Q}}_{\ell} .
$$


О ФОРМУЛАХ ДЛЯ ЧИСЛА КЛАССОВ ВЕЩЕСТВЕННЫХ АБЕЛЕВЫХ ПОЛЕЙ 99

Если $\chi \in \widehat{\mathcal{G}}$ - примитивный характер Дирихле кондуктора $f>1$, то, как и в $[15$, раздел 2], мы полагаем

$$
u(\chi)=\sum_{\substack{a \bmod f \\(a, f)=1}} \chi(a) u\left(\frac{a}{f}\right) .
$$

Пусть

$$
\omega=\sum_{\substack{\chi \in \widehat{\mathcal{G}} \\ \chi \neq 1}} u(\bar{\chi}) e_{\chi} \in \mathbf{A}_{K} .
$$

ПРЕДЛОЖЕнИЕ 8.7. Пусть $V$ определено, как выще, $и \mathbf{U}=\overline{\mathcal{O}} \cdot \mathcal{U} \subset \mathbf{A}_{K}$. Тогда

$$
\left(1-e_{1}\right) V=\omega \cdot \mathbf{U}
$$

Это предложение может быть доказано теми же рассуждениями, что и предложение 2.1 из [15].

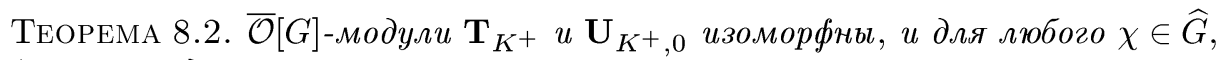
$\chi \neq 1$, справедливо равенство

$$
e_{\chi} \mathbf{T}_{K^{+}}=\frac{1}{2} a(\chi) e_{\chi} \mathbf{U}_{K^{+}},
$$

где а $(\chi)$ задано формулой (7.19).

ДоКАЗАТЕЛЬСТво. Заметим, что (8.11) индуцирует изоморфизм

$$
l: \mathbf{T}_{K^{+}} \cong\left(1-e_{1}\right) V,
$$

причем $\mathrm{Sp}_{J} \cdot l=\mathrm{id}$. С другой стороны, для нечетных $\chi$ мы имеем $u(\bar{\chi})=0$, поэтому $\omega \mathbf{U}=\omega e^{+} \mathbf{U}$, где $e^{+}=\frac{1+j}{2}$. В силу (8.7) мы получаем $\omega \mathbf{U}=\omega e^{+} \mathbf{U}_{0}$. Отображение $\operatorname{Sp}_{J}$ изоморфно отображает $l\left(\left(1-e_{1}\right) V\right)$ на $\mathbf{T}_{K^{+}}$и $\omega \mathbf{U}$ на $\omega \mathbf{U}_{K^{+}}$. Следовательно, в $\mathbf{A}_{K^{+}}$вьполняется равенство

$$
\mathbf{T}_{K^{+}}=\omega \mathbf{U}_{K^{+}} .
$$

Если $\chi \neq 1$ - четный характер группы $\mathcal{G}$, то $\chi$ можно рассматривать как характер $G$, и тогда из (7.19) и (8.11) следует, что $\frac{1}{2} a(\chi)=\mu u(\bar{\chi})$, где $\mu-$ некоторый корень из единицы, зависяший от выбора $\varepsilon_{\chi}$ в $(7.19)$. Следствие из теоремы 7.3 показывает, что $u(\bar{\chi}) \neq 0$ для любого четного $\chi \in \widehat{\mathcal{G}}, \chi \neq 1$, поэтому (8.12) определяет изоморфизм $\overline{\mathcal{O}}[G]$-модулей

$$
\mathbf{T}_{K^{+}} \cong \mathbf{U}_{K^{+}, 0}
$$

и для любого такого $\chi$

$$
e_{\chi} \mathbf{T}_{K^{+}}=\left(e_{\chi} \omega\right) e_{\chi} \mathbf{U}_{K^{+}}=u(\bar{\chi}) e_{\chi} \mathbf{U}_{K^{+}}=\frac{1}{2} a(\chi) e_{\chi} \mathbf{U}_{K^{+}} .
$$


ТЕорема 8.3 (теорема 1 введения). Пусть $K^{+}$- максимальное вещественное подполе кругового поля $K=\mathbf{Q}\left(\varepsilon_{m}\right)$ кондуктора $m$. Пусть $U\left(K^{+}\right)$- группа единич поля $K^{+}$и $C^{+}=C \cap U\left(K^{+}\right)$, где $C$ - группа круговых единии, поля $K$, определенная выше. Тогда

и для любого $\varphi \in \Phi$

$$
\left|\mathrm{Cl}\left(K^{+}\right)_{\ell}\right|=\left\{\begin{array}{lll}
{\left[U\left(K^{+}\right)[\ell]: C^{+}[\ell]\right]} & \text { npu } & \ell \neq 2, \\
2^{-b}\left[U\left(K^{+}\right)[\ell]: C^{+}[\ell]\right] & \text { npu } & \ell=2,
\end{array}\right.
$$

$$
\left|\mathrm{Cl}\left(K^{+}\right)_{\ell, \varphi}\right|=\left\{\begin{array}{lll}
{\left[U\left(K^{+}\right)[\ell]_{\varphi}: C^{+}[\ell]_{\varphi}\right]} & \text { nрu } \quad \ell \neq 2, & \\
{\left[U\left(K^{+}\right)[\ell]_{\varphi}: C^{+}[\ell]_{\varphi}\right]} & \text { npu } \quad \ell=2, \quad \varphi \neq \varphi_{0}, \\
2^{-b}\left[U\left(K^{+}\right)[\ell]_{\varphi_{0}}: C^{+}[\ell]_{\varphi_{0}}\right] & \text { nрu } \quad \ell=2, \quad \varphi=\varphi_{0},
\end{array}\right.
$$

где b определяется следующим образом. Если $g$ - число различных простых делителей $m$, то $b=0$ при $g=1 u b=2^{g-2}+1-g$ при $g>1$.

ДокАЗАТЕЛЬСТво. Теорема 8.2 показывает, что определенная выше пара модулей $\mathcal{U}_{K^{+}}, \mathcal{T}_{K^{+}}$удовлетворяет всем условиям теоремы 7.4. Тогда, применяя эту теорему, мы получаем

$$
\begin{aligned}
\left|\mathrm{Cl}\left(K^{+}\right)_{\ell}\right| & =\left(\mathcal{R}_{K^{+}, 0}: \mathcal{U}_{K^{+}, 0}\right)^{-1}\left[\mathcal{T}_{K^{+}}: C_{K^{+}}\right]^{-1}\left[\bar{U}\left(K^{+}\right)[\ell]: C_{K^{+}}\right] \\
\left|\mathrm{Cl}\left(K^{+}\right)_{\ell, \varphi}\right| & =\left(\mathcal{R}_{K^{+}, 0, \varphi}: \mathcal{U}_{K^{+}, 0, \varphi}\right)^{-1}\left[\mathcal{T}_{K^{+}, \varphi}: C_{K^{+}, \varphi}\right]^{-1}\left[\bar{U}\left(K^{+}\right)[\ell]_{\varphi}: C_{K^{+}, \varphi}\right] .
\end{aligned}
$$

Согласно определению мы имеем $C_{K^{+}}=\bar{U}\left(K^{+}\right)[\ell] \cap \mathcal{T}_{K^{+}}=C^{+}[\ell] /\{ \pm 1\}$, поэтому $\bar{U}\left(K^{+}\right)[\ell] / C_{K^{+}} \cong U\left(K^{+}\right)[\ell] / C^{+}[\ell]$.

Индекс $Q=\left[\bar{C}[\ell]:\left(C^{+}[\ell] /\{ \pm 1\}\right)\right]$ - это так называемый "индекс единиц". Хорошо известно (см., например, [15, раздел 1]), что $Q=1$ при $g=1$ и $Q=2$ при $g \geqslant 2$. Сопоставляя это с леммой 8.7 , мы получаем

$$
\begin{aligned}
& \nu_{\ell}\left(\left[\mathcal{T}_{K^{+}}: C_{K^{+}}\right]\right)=\left\{\begin{array}{lll}
\nu_{\ell}\left(2^{-g}|\mathcal{G}|\right) & \text { при } & g=1, \\
\nu_{\ell}\left(2^{1-g}|\mathcal{G}|\right) & \text { при } & g>1,
\end{array}\right. \\
& {\left[\mathcal{T}_{K^{+}, \varphi}: C_{K^{+}, \varphi}\right]= \begin{cases}1 & \text { при } \varphi \neq \varphi_{0}, \\
{\left[\mathcal{T}_{K^{+}}: C_{K^{+}}\right]} & \text {при } \varphi=\varphi_{0} .\end{cases} }
\end{aligned}
$$

Мы имеем $\mathcal{R}_{K^{+}, 0}=\mathcal{R}_{0}^{J}$, поэтому индексы $\left(\mathcal{R}_{K^{+}, 0}: \mathcal{U}_{K^{+}, 0}\right)$ и $\left(\mathcal{R}_{K^{+}, 0, \varphi}: \mathcal{U}_{K^{+}, 0, \varphi}\right)$ определяются из предложения 8.6. Сопоставляя предложение 8.6 с формулами (8.13) и (8.14), мы получаем утверждение теоремы.

Следуюший результат является непосредственным следствием теоремы 8.3.

TeOpema 8.4. Пусть $K u K^{+}$me жеe, что и в теореме 8.3. Пусть $\ell-$ фиксированное простое число и $k$-некоторое подполе поля $K^{+}$, такое что степень $\left[K^{+}: k\right]$ взаимно проста $c \ell$. Пусть $U(k)$ - группа единии, поля $k u$ $C^{+}(k)=C^{+} \cap U(k)$. Пусть $\varphi \in \Phi-$ некоторый характер группь $G=G\left(K^{+} / \mathbf{Q}\right)$ такой, что ограничение $\varphi$ на $G\left(K^{+} / k\right)$ тривиально (таким образом, $\varphi$ можно рассматривать как характер группы $G(k / \mathbf{Q}))$. Тогда

$$
\begin{aligned}
& \left|\mathrm{Cl}(k)_{\ell}\right|=\left\{\begin{array}{lll}
{\left[U(k)[\ell]: C^{+}(k)[\ell]\right]} & \text { nрu } & \ell \neq 2, \\
2^{-b}\left[U(k)[\ell]: C^{+}(k)[\ell]\right] & \text { nрu } & \ell=2,
\end{array}\right. \\
& \left|\mathrm{Cl}(k)_{\ell, \varphi}\right|=\left\{\begin{array}{lll}
{\left[U(k)[\ell]_{\varphi}: C^{+}(k)[\ell]_{\varphi}\right]} & \text { nрu } \quad \ell \neq 2, & \\
{\left[U(k)[\ell]_{\varphi}: C^{+}(k)[\ell]_{\varphi}\right]} & \text { nрu } \quad \ell=2, & \varphi \neq \varphi_{0}, \\
2^{-b}\left[U(k)[\ell]_{\varphi_{0}}: C^{+}(k)[\ell]_{\varphi_{0}}\right] & \text { nрu } \quad \ell=2, & \varphi=\varphi_{0},
\end{array}\right.
\end{aligned}
$$

где $b$ то же, что и в теореме 8.3. 
О ФОРМУЛАХ ДЛЯ ЧИСЛА КЛАССОВ ВЕЩЕСТВЕННЫХ АБЕЛЕВЫХ ПОЛЕЙ 101

ДокАЗАТЕЛЬСтво. Если $\varphi$ удовлетворяет условиям, указанным выше, то $\mathrm{Cl}(k)_{\ell, \varphi}=\mathrm{Cl}\left(K^{+}\right)_{\ell, \varphi}, U(k)[\ell]_{\varphi}=U\left(K^{+}\right)[\ell]_{\varphi}$ и $C^{+}(k)[\ell]_{\varphi}=C^{+}[\ell]_{\varphi}$.

\section{§9. Доказательство теоремы 2 и некоторые другие результаты}

Пусть $k$ - произвольное вещественное абелево поле. Если $k$ имеет кондуктор $m$, то $k$ является подполем кругового поля $K=K_{m}=\mathbf{Q}\left(\varepsilon_{m}\right)$. Более того, $k$ является подполем максимального вешественного подполя $K^{+}=K_{m}^{+}$поля $K_{m}$. Положим $H=G\left(K^{+} / k\right), G=G(k / \mathbf{Q})$ и $\mathcal{R}_{k}=\mathbf{Z}_{\ell}[G]$. В этом параграфе мы дадим определение модулей $\mathcal{U}_{k}$ и $\mathcal{T}_{k}$ для произвольного вещественного абелева $k$. Мы можем сделать это по крайней мере тремя способами.

ОПРЕДЕЛЕНИЕ 1. Положим $\mathcal{U}_{k}^{(1)}:=\pi_{H}\left(\mathcal{U}_{K^{+}}\right), \mathcal{T}_{k}^{(1)}:=N_{H} \mathcal{T}_{K^{+}}$и $C_{k}^{(1)}:=$ $N_{H} C_{K^{+}}$, где $\pi_{H}-$ естественная проекция $\mathbf{Q}_{\ell}\left[G\left(K^{+} / \mathbf{Q}\right)\right] \rightarrow \mathbf{Q}_{\ell}[G]$ и $N_{H}$ - отображение $\bar{U}\left(K^{+}\right) \otimes_{\mathbf{z}} \mathbf{Q}_{\ell} \rightarrow \bar{U}(k) \otimes_{\mathbf{z}} \mathbf{Q}_{\ell}$, индуцированное норменным отображением $N_{H}: U\left(K^{+}\right) \rightarrow U(k)$.

Для произвольного натурального $n \mid m$ мы положим, как и в [16], $k_{n}=K_{n} \cap k$, где $K_{n}=\mathbf{Q}\left(\varepsilon_{n}\right)$ - круговое поле кондуктора $n$. Заметим, что любое $k_{n}$ является подполем максимального вешественного подполя $K_{n}^{+}$поля $K_{n}$. Пусть $\pi_{n}$ - естественная проекция $\mathbf{Q}_{\ell}\left[G\left(K_{n}^{+} / \mathbf{Q}\right)\right] \rightarrow \mathbf{Q}_{\ell}\left[G\left(k_{n} / \mathbf{Q}\right)\right]$ и $i_{n}: \mathbf{Q}_{\ell}\left[G\left(k_{n} / \mathbf{Q}\right)\right] \rightarrow \mathbf{Q}_{\ell}[G]-$ отображение, определенное формулой $i_{n}(\sigma)=\sum_{\tau \in G\left(k / k_{n}\right)} \bar{\sigma} \tau$, где $\sigma \in G\left(k_{n} / \mathbf{Q}\right)$ и $\bar{\sigma}$ - некоторый представитель $\sigma$ в $G$. С другой стороны, для любого $n \mid m$ имеются норменное отображение $N_{K_{n}^{+} / k_{n}}: \bar{U}\left(K_{n}^{+}\right) \otimes_{\mathbf{z}} \mathbf{Q}_{\ell} \rightarrow \bar{U}\left(k_{n}\right) \otimes_{\mathbf{z}} \mathbf{Q}_{\ell}$ и естественное включение $j_{n}: \bar{U}\left(k_{n}\right) \otimes_{\mathbf{z}} \mathbf{Q}_{\ell} \hookrightarrow \bar{U}(k) \otimes_{\mathbf{z}} \mathbf{Q}_{\ell}$. Данное ниже определение следует определению, данному в [16].

ОПРЕДЕЛЕНиЕ 2 . Определим $\mathcal{U}_{k}^{(2)}$ как $\mathcal{R}_{k}$-подмодуль в $\mathbf{Q}_{\ell}[G]$, порожденный группами $i_{n} \circ \pi_{n}\left(\mathcal{U}_{K_{n}^{+}}\right)$для всех $n \mid m$. Определим $\mathcal{T}_{k}^{(2)}$ как $\mathcal{R}_{k}$-подмодуль в $\bar{U}(k) \otimes \mathbf{z} \mathbf{Q}_{\ell}$, порожденный группами $j_{n} \circ N_{K_{n}^{+} / k_{n}}\left(\mathcal{T}_{K_{n}^{+}}\right)$для всех $n \mid m$. Определим $C_{k}^{(2)}$ как $\mathcal{R}_{k}$-подмодуль в $\bar{U}(k)[\ell]$, порожденньй группами $j_{n} \circ N_{K_{n} / k_{n}} C\left(K_{n}\right)$, где $C\left(K_{n}\right)$ - группа круговых единиц, определенная во введении.

ЗАмечАниЕ. Заметим, что $\mathcal{U}_{k}^{(2)} \supseteq i_{1} \circ \pi\left(\mathcal{U}_{K_{1}}^{+}\right)=s(G) \mathbf{Z}_{\ell}$. Следовательно,

$$
\mathcal{U}_{k}^{(2)}=\mathcal{U}_{k, 0}^{(2)}+s(G) \mathbf{Z}_{\ell} .
$$

ОПРЕДЕЛЕНИЕ 3 . Положим $\mathcal{U}_{k}^{(3)}:=i_{m}^{-1}\left(\mathcal{U}_{K_{m}^{+}}^{H}\right) \subset \mathbf{Q}_{\ell}[G], \mathcal{T}_{k}^{(3)}:=j_{m}^{-1}\left(\mathcal{T}_{K_{m}^{+}}^{H}\right) \subset$ $\bar{U}(k) \otimes_{\mathbf{z}} \mathbf{Q}_{\ell}$ и $C_{k}^{(3)}:=C_{K^{+}}^{H}=\bar{U}(k) \cap C_{K^{+}}$.

В дальнейшем мы будем обозначать модуль $\mathcal{U} \subset \mathbf{Q}_{\ell}\left[G\left(K_{m} / \mathbf{Q}\right)\right]$, введенный в $\S 8$, через $\mathcal{U}_{m}$, если мы хотим явно указать его зависимость от $K_{m}$. Для $i=1,2,3$ положим $\mathbf{U}_{k}^{(i)}=\overline{\mathcal{O}} \cdot \mathcal{U}_{k}^{(i)} \subset \mathbf{A}_{k}$ и $\mathbf{T}_{k}^{(i)}=\overline{\mathcal{O}} \cdot \log \left(\mathcal{T}_{k}^{(i)}\right)$, где отображение $\log : \mathcal{T}_{k}^{(i)} \hookrightarrow$ $\mathbf{A}_{k}$, определено, как в (8.10).

ПрЕДЛОЖЕНИЕ 9.1. Пусть $\omega \in \mathbf{A}_{K_{m}}$ имеет тот же смицсл, что и $в$ предложении 8.7. Тогда для $i=1,2,3$ справедливы равенства

$$
\mathbf{T}_{k}^{(i)}=\omega \mathbf{U}_{k}^{(i)}
$$


ДокАЗАТЕЛЬСТВо. В силу (8.12) мы имеем $\mathbf{T}_{K^{+}}=\omega \mathbf{U}_{K^{+}}$, где $K^{+}=K_{m}^{+}$. Из определения 1 и тождества $\log \cdot N_{H}=\mathrm{Sp}_{H} \cdot \log$ следует, что $\mathbf{T}_{k}^{(1)}=\operatorname{Sp}_{H} \mathbf{T}_{K^{+}}$и $\mathbf{U}_{k}^{(1)}=\operatorname{Sp}_{H} \mathbf{U}_{K^{+}}$. Таким образом, $\mathbf{T}_{k}^{(1)}=\operatorname{Sp}_{H}\left(\omega \mathbf{U}_{K^{+}}\right)=\omega \cdot \operatorname{Sp}_{H}\left(\mathbf{U}_{K^{+}}\right)=\omega \mathbf{U}_{k}^{(1)}$. Это доказывает наше предложение для $i=1$.

Поскольку умножение на $\omega$ в (8.12) индуцирует изоморфизм $\mathbf{T}_{K^{+}} \cong \mathbf{U}_{K^{+}, 0}$, мы получаем

$$
\mathbf{T}_{k}^{(3)}=\mathbf{T}_{K^{+}}^{H}=\omega \mathbf{U}_{K^{+}}^{H}=\omega \mathbf{U}_{k}^{(3)} .
$$

Это доказывает наше предложение для $i=3$.

Для $i=2$ и $n \mid m$ положим

$$
\omega_{n}=\sum_{\substack{\chi \in G\left(K_{n} / \mathbf{Q}\right) \\ \chi \neq 1}} u(\bar{\chi}) e_{\chi} \in \mathbf{A}_{K_{n}} .
$$

Применяя к полю $K_{n}$ предложение 8.7 , мы получаем

$$
\mathbf{T}_{K_{n}^{+}}=\omega_{n} \mathbf{U}_{K_{n}^{+}}
$$

Если $x \in \mathbf{A}_{K}^{G\left(K / K_{n}\right)}=\mathbf{A}_{K_{n}}$, то для любого характера $\chi \in \widehat{G}$, имеюшего нетривиальное ограничение на $G\left(K / K_{n}\right)$, мы имеем $e_{\chi} \cdot x=0$. Тогда $\omega_{n} \cdot x=\omega \cdot x$ для любого $x \in \mathbf{A}_{K_{n}}$ и, следовательно, $\mathbf{T}_{K_{n}^{+}}=\omega \mathbf{U}_{K_{n}^{+}}$для любого $n \mid m$. Из определения 2 следует, что $\mathbf{T}_{k}^{(2)}$ порождается в $\mathbf{A}_{k}$ группами $\operatorname{Sp}_{K_{n} / k_{n}}\left(\mathbf{T}_{K_{n}^{+}}\right)$для всех $n \mid m$, а $\mathbf{U}_{k}^{(2)}$ порождается в $\mathbf{A}_{k}$ группами $\operatorname{Sp}_{K_{n} / k_{n}}\left(\mathbf{U}_{K_{n}^{+}}\right)$для всех $n \mid m$. Поскольку $\omega \cdot \operatorname{Sp}_{K_{n} / k_{n}}(x)=\operatorname{Sp}_{K_{n} / k_{n}}(\omega \cdot x)$ для любого $x \in \mathbf{A}_{K_{n}}$, мы получаем

$$
\mathbf{T}_{k}^{(2)}=\omega \mathbf{U}_{k}^{(2)} .
$$

Предложение доказано.

В качестве непосредственного следствия из теоремы 7.4 и предложения 9.1 мы получаем следующее утверждение.

Теорема 9.1. Пусть $k$-вещественное абелево поле. Тогда для $i=1,2,3$ и любого $\varphi \in \Phi$ мы имеем

$$
\left|\mathrm{Cl}(k)_{\ell}\right|=\left(\mathcal{R}_{k, 0}: \mathcal{U}_{k, 0}^{(i)}\right)^{-1}\left[\mathcal{T}_{k}^{(i)}: C_{k}^{(i)}\right]^{-1}\left[\bar{U}(k)[\ell]: C_{k}^{(i)}\right]
$$

$u$

$$
\left|\mathrm{Cl}(k)_{\ell, \varphi}\right|=\left(\mathcal{R}_{k, 0, \varphi}: \mathcal{U}_{k, 0, \varphi}^{(i)}\right)^{-1}\left[\mathcal{T}_{k, \varphi}^{(i)}: C_{k, \varphi}^{(i)}\right]^{-1}\left[\bar{U}(k)[\ell]_{\varphi}: C_{k, \varphi}^{(i)}\right]
$$

ПРЕДЛОЖЕНИЕ 9.2. Индекс $\left(\mathcal{R}_{k, 0}: \mathcal{U}_{k, 0}^{(i)}\right)$ определяется равенством

$$
\left(R_{k, 0}: \mathcal{U}_{k, 0}^{(i)}\right)=\ell^{c}\left(\mathcal{R}_{k}: \mathcal{U}_{k}^{(i)}\right)
$$

zде

$$
c=\left\{\begin{array}{lll}
\nu_{\ell}([K: \mathbf{Q}]) & n p u & i=1, \\
\nu_{\ell}([k: \mathbf{Q}]) & n p u & i=2,3
\end{array}\right.
$$


О ФОРМУЛАХ ДЛЯ ЧИСЛА КЛАССОВ ВЕЩЕСТВЕННЫХ АБЕЛЕВЫХ ПОЛЕЙ 103

ДокАЗАТЕЛЬСТво. Для $i=1,3$ наше утверждение непосредственно следует из (7.5) и (8.7). При $i=2$ наше утверждение следует из (7.5) и (9.1).

ПРЕДЛОЖЕНИЕ 9.3. Для вещественного абелева $k$ пусть $U \subset \mathbf{Q}[G]-$ модуль, определенный Синноттом в [16]. Тогда

$$
\mathcal{U}_{k}^{(2)}=U[\ell]
$$

ДокаЗАТЕЛЬСтво. Как и в $\S 8$, положим $\bar{m}=\prod_{p \mid m} p$. Пусть $T_{p}$ (соответственно $\left.T_{p}(k)\right)$ - подгруппа инерции точки $p$ в $\mathcal{G}=G\left(K_{m} / \mathbf{Q}\right)$ (соответственно в $G=G(k / \mathbf{Q}))$. Как было показано в доказательстве предложения 5.1 из [15], модуль $\mathcal{U}_{m}$ порождается как $\mathbf{Z}_{\ell}[\mathcal{G}]$-модуль элементами

$$
p_{m, r}:=\prod_{p \mid r} s\left(T_{p}\right) \prod_{p \mid \bar{m} / r}\left(1-\bar{\sigma}_{p}\right), \quad 1 \leqslant r \leqslant \bar{m}, \quad r \mid m .
$$

Для $p \mid m$ положим $(p, k)^{*}=\lambda_{p}^{-1} e_{p}^{\prime}$, где $e_{p}^{\prime}=\left|T_{p}(k)\right|^{-1} \sum_{\sigma \in T_{p}(k)} \sigma$ и $\lambda_{p}-$ любой элемент группы $G$ такой, что $\lambda_{p} \bmod T_{p}(k)$ является автоморфизмом $\Phi$ робениуса, соответствующим $p$, в группе $G / T_{p}(k)$. Для $r \mid \bar{m}$ обозначим через $T_{r}(k)$ композит в $G$ подгрупп инерции $T_{p}(k)$ для всех $p$, делящих $r$. В [16, предложение 2.3$]$ было доказано, что модуль $U$ порождается как $\mathbf{Z}[G]$-модуль элементами

$$
q_{r}:=s\left(T_{r}(k)\right) \prod_{p \mid \bar{m} / r}\left(1-(p, k)^{*}\right),
$$

где $r$ пробегает все положительные делители $\bar{m}$.

Пусть $r|\bar{n}| \bar{m}$. Мы докажем, что $i_{n} \circ \pi_{n}\left(p_{n}, r\right) \in U$. Пусть $T_{r}\left(k_{n}\right)$ (соответственно $\left.T_{r}\left(K_{n}\right)\right)$ - композит в $G=G\left(k_{n} / \mathbf{Q}\right)\left(\right.$ соответственно в $\left.G\left(K_{n} / \mathbf{Q}\right)\right)$ подгрупп инерции $T_{p}\left(k_{n}\right)$ (соответственно $T_{p}\left(K_{n}\right)$ ) для всех $p$, делящих $r$. Поскольку отображение $\pi_{n}$ является гомоморфизмом колец и $\pi_{n}\left(\bar{\sigma}_{p}\right)=\left(p, k_{n}\right)^{*}$, мы получаем

$$
\pi_{n}\left(p_{n, r}\right)=\frac{\left|T_{r}\left(K_{n}\right)\right|}{\left|T_{r}\left(k_{n}\right)\right|} s\left(T_{r}\left(k_{n}\right)\right) \prod_{p \mid \bar{n} / r}\left(1-(p, k)^{*}\right) .
$$

Положим $r_{1}=r \bar{m} / \bar{n}$, т.е. $\bar{m} / r_{1}=\bar{n} / r$. Пусть $\pi^{(n)}$ - естественное отображение $\mathbf{Q}[G] \rightarrow \mathbf{Q}\left[G\left(k_{n} / \mathbf{Q}\right)\right]$. Тогда, рассуждая, как и прежде, и учитывая, что $\pi^{(n)}\left(T_{r_{1}}(k)\right)=T_{r}\left(k_{n}\right)$, мы получаем

$$
\pi_{n}\left(q_{r_{1}}\right)=\frac{\left|T_{r_{1}}(k)\right|}{\left.\mid T_{r} k_{n}\right) \mid} s\left(T_{r}\left(k_{n}\right)\right) \prod_{p \mid \bar{n} / r}\left(1-(p, k)^{*}\right)=\frac{\left|T_{r_{1}}(k)\right|}{\left|T_{r}\left(K_{n}\right)\right|} \pi_{n}\left(p_{n, r}\right) .
$$

Рассмотрим точную последовательность

$$
0 \longrightarrow G\left(k / k_{n}\right) \cap T_{r_{1}}(k) \longrightarrow T_{r_{1}}(k) \longrightarrow T_{r}\left(k_{n}\right) \longrightarrow 0 .
$$

Мы имеем

$$
\left|G\left(k / k_{n}\right) \cap T_{r_{1}}(k)\right|=\frac{\left|T_{r_{1}}(k)\right|}{\left|T_{r}\left(k_{n}\right)\right|} .
$$


Пусть $S \subset G\left(k / k_{n}\right)$ - множество представителей для $G\left(k / k_{n}\right) /\left(G\left(k / k_{n}\right) \cap T_{r_{1}}(k)\right)$. Тогда

$$
i_{n} \circ \pi^{(n)}\left(q_{r_{1}}\right)=\operatorname{Sp}_{k / k_{n}}\left(q_{r_{1}}\right)=\frac{\left|T_{r_{1}}(k)\right|}{\left|T_{r}\left(k_{n}\right)\right|} \sum_{\sigma \in S} \sigma\left(q_{r_{1}}\right) .
$$

Таким образом, из (9.2) следует, что

$$
i_{n} \circ \pi^{(n)}\left(p_{n, r}\right)=\frac{\left|T_{r}\left(K_{n}\right)\right|}{\left|T_{r_{1}}(k)\right|} \operatorname{Sp}_{k / k_{n}}\left(q_{r_{1}}\right)=\frac{\left|T_{r}\left(K_{n}\right)\right|}{\left|T_{r}\left(k_{n}\right)\right|} \sum_{\sigma \in S} \sigma\left(q_{r_{1}}\right) .
$$

Тогда $i_{n} \circ \pi_{n}\left(p_{n, r}\right) \in U$ для любого $n|m, r| \bar{n}$. Следовательно, $\mathcal{U}_{k}^{(2)} \subseteq U[\ell]$.

Теперь мы докажем, что $q_{2} \in \mathcal{U}_{k}^{(2)}$ для любого $r \mid \bar{m}$. Положим $m=a b$, где $(b, r)=1$ и простое $p$ делит $a$ тогда и только тогда, когда $p$ делит $r$ (таким образом, $\bar{a}=r)$. Отметим, что $T_{r}(k)=G\left(k / k_{b}\right)$. Рассмотрим произведение

$$
p_{b, 1}=\prod_{p \mid \bar{m} / r}\left(1-\bar{\sigma}_{p}\right)
$$

Мы имеем

$$
\pi_{b}\left(p_{b, 1}\right)=\prod_{p \mid \bar{m} / r}\left(1-\left(p, k_{b}\right)^{*}\right) .
$$

Поскольку $q_{r} \in \mathbf{Q}[G]^{T_{r}(k)}$, мы получаем $q_{r}=\left|T_{r}(k)\right|^{-1} \cdot i_{b} \circ \pi^{(b)}\left(q_{r}\right)$. Отметим, что

$$
\pi^{(b)}\left(q_{r}\right)=\left|T_{r}(k)\right| \cdot \prod_{p \mid \bar{m} / r}\left(1-\left(p, k_{b}\right)^{*}\right) .
$$

Поэтому $\pi_{b}\left(p_{b, 1}\right)=\left|T_{r}(k)\right|^{-1} \pi^{(b)}\left(q_{r}\right)$. Следовательно,

$$
q_{r}=i_{b} \circ \pi_{b}\left(p_{b, 1}\right) .
$$

Этим доказано, что $\mathcal{U}_{k}^{(2)} \supseteq U[\ell]$. Предложение доказано.

ПредЛОЖениЕ 9.4. Группа $\left(\mathcal{T}_{k}^{(2)}\right)^{2}$, әде $\mathcal{T}_{k}^{(2)}$ - группа из определения 2, coвnадаeт с әруппой $\left(1-e_{G}\right)(D /\{ \pm 1\})$, где $e_{G}=|G|^{-1} \sum_{\sigma \in G} \sigma$ u $D-$ группа круговых чисел поля $k$, определенная Синноттом в [16, раздел 4].

Группа $C_{k}^{(2)}$ определения 2 совпадает с $C /\{ \pm 1\}$, где $C$ - группа круговых единии поля $k$, определенная в [16, раздел 4$]$.

ДокАЗАТельство. Группа $D$ определяется следующим образом. Пусть $n-$ любое целое число, большее 1, и $a$ - любое целое число, не делящееся на $n$. Тогда число $N_{K_{n} / k_{n}}\left(1-\varepsilon_{n}^{a}\right)$ принадлежит полю $k$. Синнотт определяет группу круговых чисел $D$ поля $k$ как группу, порожденную в $k^{*}$ элементом -1 и всеми элементами вида $N_{K_{n} / k_{n}}\left(1-\varepsilon_{n}^{a}\right)$. Затем, круговые единицы $C$ определяются равенством $C=$ $U(k) \cap D$. Отметим, что любой элемент $x \in D$ является единицей тогда и только тогда, когда $N_{k / \mathbf{Q}}(x)= \pm 1$. Следовательно, $C /\{ \pm 1\}=(D /\{ \pm 1\})_{0}$.

Лемма 9.1. Пусть $D_{1}$ - подгруппа группы $D$, порожденная әлементом - 1 и всеми әлементами вида $N_{K_{n} / k_{n}}\left(1-\varepsilon_{n}^{a}\right)$ для $n \mid m$, а $D_{2}-$ подгруппа в $\mathbf{Q}^{*}$, порожденная всеми простыми числами $q \backslash$ т. Тогда существует разложение в прямое произведение $D \cong D_{1} \times D_{2}$. 
ДокАЗАТЕЛЬСТво. В [16] было показано, что $D \supset \mathbf{Q}^{*}$, следовательно, $D_{2} \subset D$. Очевидно, что $D_{1} \subset D$ и $D_{1} \cap D_{2}=\{1\}$. Зафиксируем некоторое $n=n_{1} n_{2}$, где $\left(m, n_{2}\right)=1$ и произвольное простое число $p$ может делить $n_{1}$ только в том случае, когда $p \mid m$. Тогда $K_{n}=K_{n_{1}} \cdot K_{n_{2}}$ и $K_{n_{2}} \cap k=\mathbf{Q}$.

Если $n_{1}=1$, т.е. $(n, m)=1$, то $k_{n}=\mathbf{Q}$, и, учитывая, что норма $N_{K_{n} / k_{n}}(x)$ всюду положительна для любого $x \in K_{n}$, мы получаем $N_{K_{n} / k_{n}}\left(1-\varepsilon_{n}^{a}\right) \in D_{2}$.

Если $n_{2}=1$, то, полагая $E=K_{n} \cap K_{m}$, мы получаем $N_{K_{n} / k_{n}}=N_{E / k_{n}}$ 。 $N_{K_{n} / E}$. Прямое вычисление показывает, что $N_{K_{n} / E}\left(1-\varepsilon_{n}^{a}\right) \in P(E)$, следовательно, $N_{K_{n} / k}\left(1-\varepsilon_{n}^{a}\right) \in D_{1}$.

Если $n_{1} \neq 1, n_{2} \neq 1$, то, рассуждая тем же образом и полагая $E=K_{n} \cap K_{m}$, мы получаем $N_{K_{n} / k_{n}}\left(1-\varepsilon_{n}^{a}\right)=N_{E / k_{n}} \circ N_{K_{n} / E}\left(1-\varepsilon_{n}^{a}\right)$. Поскольку в этом случае $\left(1-\varepsilon_{n}^{a}\right)$ является единицей, мы видим, что $N_{K_{n} / E}\left(1-\varepsilon_{n}^{a}\right) \in P(E)$, следовательно, $N_{K_{n} / k}\left(1-\varepsilon_{n}^{a}\right) \in D_{1}$. Лемма доказана.

Из леммы 9.1 следует, что $\left(1-e_{G}\right)(D /\{ \pm 1\})=\left(1-e_{G}\right)\left(D_{1} /\{ \pm 1\}\right)$. Без труда проверяется, что группа $\left(1-e_{G}\right) D_{1}$ порождается группами $\left(1-e_{n}\right) \bar{P}_{n}$ для всех $n \mid m$, где $P_{n}$ - группа круговых чисел поля $K_{n}, \bar{P}_{n}=P_{n} / \mu\left(K_{n}\right)$ и $e_{n}=\mid G\left(K_{n} /\left.\mathbf{Q}\right|^{-1} \sum_{\sigma \in G\left(K_{n} / \mathbf{Q}\right)} \sigma\right.$. Согласно определению $\mathcal{T}_{K_{n}^{+}}=\left(1-e_{n}\right) \bar{P}_{n}$. Учитывая, что мы использовали отображение $N_{K_{n}^{+} / k_{n}}$ в определении группы $\mathcal{T}_{k}^{(2)}$ и отображение $N_{K_{n} / k}$ в определении группы $D$, мы получаем, что $\left(\mathcal{T}_{k}^{(2)}\right)^{2}=(1-$ $\left.e_{G}\right)(D /\{ \pm 1\})$

Чтобы доказать последнее утверждение предложения, заметим, что включение $C_{k}^{(2)} \subseteq C /\{ \pm 1\}=(D /\{ \pm 1\})_{0}$ очевидно. Предположим, что $x \in D$ и $x \bmod$ $\{ \pm 1\} \in(D /\{ \pm 1\})_{0}$. Тогда $x$ можно представить в виде $x=u \cdot \prod_{p \mid m} A_{p}$, где $u \in$ $C_{k}^{(2)}$ и $A_{p}$ определяются следующим образом. Пусть $p^{e}$ - максимальная степень $p$, делящая $m$. Для $1 \leqslant j \leqslant e$ положим $K(j)=K_{p^{j}}$ и $k(j)=k_{p^{j}}$. Тогда $A_{p}$ имеет вид $A_{p}=\prod_{j=1}^{e} B_{j}$, где $B_{j}=N_{K(j) / k(j)}\left(b_{j}\right)$ для некоторых $b_{j} \in P(K(j))$. Поскольку элементы $A_{p}$ взаимно просты в $k$, мы получаем, что $x$ является единицей тогда и только тогда, когда $A_{p}$ является единищей для любого $p \mid m$. Группа $G\left(k_{p^{e}} / \mathbf{Q}\right)$ циклическая (группа $G\left(k_{p^{e}}^{+} / \mathbf{Q}\right)$ циклическая в случае $\left.p=2\right)$, поэтому, учитывая, что $p^{e}$ - точная степень $p$, делящая кондуктор поля $k$, мы получаем, что степень $\left[K_{p^{j}}: k_{p^{j}}\right]$ не зависит от $j$ для $1 \leqslant j \leqslant e$. Следовательно, для любого $a \in K_{p^{e}}$ мы имеем

$$
N_{k(e) / k(j)} \circ N_{K(e) / k(e)}(a)=N_{K(j) / k(j)} \circ N_{K(e) / K(j)}(a) .
$$

Норма $N_{K(e) / K(j)}$ отображает $P\left(K_{p^{e}}\right)$ на $P\left(K_{p^{j}}\right)$. Следовательно, $A_{p}$ имеет вид $A_{p}=N_{K(e) / k(e)}\left(c_{p}\right)$ для некоторого $c_{p} \in P\left(K_{p e}\right)$. Очевидно, что $A_{p}$ является единищей тогда и только тогда, когда $c_{p}$ является единищей, т.е. когда $c_{p} \in C\left(K_{p^{e}}\right)$. Следовательно, $A_{p} \in C_{k}^{(2)}$. Это доказывает включение $C_{k}^{(2)} \supseteq C /\{ \pm 1\}$. Предложение доказано.

Для $\varphi \in \Phi$ положим $d=|G|=[k: \mathbf{Q}]$ и $d_{\varphi}=\operatorname{dim}_{\mathbf{Q}_{\ell}} \varphi=\operatorname{dim}_{\mathbf{Q}_{\ell}} \mathbf{Q}_{\ell}[G]_{\varphi}$, где $G=G(k / \mathbf{Q})$. Положим $d_{\varphi_{0}}=d_{0}$.

ПРЕДЛОЖЕнИЕ 9.5. Группа $C_{k}^{(2)}$ имеет конечный индекс в $\mathcal{T}_{k}^{(2)}$, и для лю- 
бого $\varphi \in \Phi$ мы имеем

$$
\begin{aligned}
{\left[\mathcal{T}_{k}^{(2)}: C_{k}^{(2)}\right] } & =2^{d-1} \prod_{p \mid m}\left[k_{p^{e}}: \mathbf{Q}\right], \\
{\left[\mathcal{T}_{k, \varphi}^{(2)}: C_{k, \varphi}^{(2)}\right] } & =\left\{\begin{array}{lll}
2^{d_{\varphi}} & \partial \Omega Я & \varphi \neq \varphi_{0}, \\
2^{d_{0}-1} \prod_{p \mid m}\left[k_{p^{e}}: \mathbf{Q}\right] & \partial \Omega Я & \varphi=\varphi_{0},
\end{array}\right.
\end{aligned}
$$

где произведение берется по всем простым $p$, делящим кондуктор $m$ поля $k$. Для любого такого р $p^{е}$ означает максимальную степень $p$, делящую $m$.

ДокАЗАТЕльство. В [16, предложение 4.1] было доказано, что для некоторой определенной там группы $T \cong D /\{ \pm 1\}$ мы имеем

$$
\left[\left(1-e_{G}\right) T: T_{0}\right]=\prod_{p \mid m}\left[k_{p^{e}}: \mathbf{Q}\right] .
$$

Из предложения 9.4 следует, что

$$
\left[\left(\mathcal{T}_{k}^{(2)}\right)_{\varphi}^{2}: C_{k, \varphi}^{(2)}\right]=\left[\left(1-e_{G}\right) T_{\varphi}: T_{0, \varphi}\right]
$$

где $T \cong D /\{ \pm 1\}$. Тогда утверждение нашего предложения следует из того факта, что

$$
\left[\mathcal{T}_{k, \varphi}^{(2)}:\left(\mathcal{T}_{k}^{(2)}\right)_{\varphi}^{2}\right]= \begin{cases}2^{d_{\varphi}} & \text { при } \varphi \neq \varphi_{0} \\ 2^{d_{0}-1} & \text { при } \varphi=\varphi_{0}\end{cases}
$$

Предложение доказано.

Сопоставляя теорему 9.1, предложение 9.2 и предложение 9.5 , мы получаем следуюший результат, являющийся уточнением теоремы 4.1 из [16].

ТЕОРема 9.2 (теорема 2 введения). Для любого вещественного абелева $k$ мы имеем

$$
\nu_{\ell}\left(\left[\bar{U}(k)[\ell]: C_{k}^{(2)}\right]\right)=\nu_{\ell}\left(\left|\mathrm{Cl}(k)_{\ell}\right| 2^{d-1} \frac{\prod_{p \mid m}\left[k_{p^{e}}: \mathbf{Q}\right]}{[k: \mathbf{Q}]}\left[\mathcal{R}_{k}: \mathcal{U}_{k}\right]\right) .
$$

Для любого $\varphi \in \Phi$ мы имеем

$$
\begin{aligned}
& \nu_{\ell}\left(\left[\bar{U}(k)[\ell]_{\varphi}: C_{k, \varphi}\right]\right) \\
& \quad= \begin{cases}\nu_{\ell}\left(\left|\mathrm{Cl}(k)_{\ell, \varphi}\right| 2^{d_{\varphi}}\left[\mathcal{R}_{k, \varphi}: \mathcal{U}_{k, \varphi}\right]\right) & \text { npu } \quad \varphi \neq \varphi_{0}, \\
\nu_{\ell}\left(\left|\mathrm{Cl}(k)_{\ell, \varphi_{0}}\right| 2^{d_{0}-1} \frac{\prod_{p \mid m}\left[k_{p}: \mathbf{Q}\right]}{[k: \mathbf{Q}]}\left[\mathcal{R}_{k, \varphi_{0}}: \mathcal{U}_{k, \varphi_{0}}\right]\right) & \text { npu } \quad \varphi=\varphi_{0} .\end{cases}
\end{aligned}
$$

В случае, когда $i=1,3$, автор не сумел полностью вычислить индекс $\left[\mathcal{T}_{k, \varphi}^{(i)}\right.$ : $\left.C_{k, \varphi}^{(i)}\right]$. Тем не менее, в случае $i=3$ мы имеем следующий тривиальный результат (случай $i=1$ может быть рассмотрен аналогичным образом, но этот последний случай не ведет ни к каким интересньм следствиям).

ПРЕДЛОЖЕНИЕ 9.6. Пусть $k$ - вещественное абелево поле с кондуктором m. Если $i=3$ и $\ell \neq 2$, то для любого $\varphi \in \Phi$ миц имеем

$$
\begin{aligned}
& {\left[\mathcal{T}_{k}^{(3)}: C_{k}^{(3)}\right]=\ell^{r},} \\
& {\left[\mathcal{T}_{k, \varphi}^{(3)}: C_{k, \varphi}^{(3)}\right]=\left\{\begin{array}{lll}
1 & n p u & \varphi \neq \varphi_{0}, \\
\ell^{r} & n p u & \varphi=\varphi_{0},
\end{array}\right.}
\end{aligned}
$$

где $\ell^{r}$ - некоторая степень $\ell$, делящая порядок группь $\mathcal{G}=G\left(K_{m} / \mathbf{Q}\right)$. 
ДокАЗАТЕЛЬСТво. Положим $K_{m}=K$. Тогда $\mathcal{T}_{K^{+}}^{H} / C_{K^{+}}^{H} \subseteq \mathcal{T}_{K^{+}} / C_{K^{+}}$. Заметим, что при $\ell \neq 2$ справедливы равенства $C_{K^{+}}=\bar{C}(K)[\ell]$ и $C_{K^{+}}^{H}=C_{k}^{(3)}$. Таким образом, предложение непосредственно следует из леммы 8.7.

ТЕОРемА 9.3. Для любого вещественного абелева поля $k$ и для $\ell \neq 2$ мы имеем

$$
\left|\mathrm{Cl}(k)_{\ell}\right|=\left(\mathcal{R}_{k}: \mathcal{U}_{k}^{(3)}\right)^{-1} \ell^{r-c}\left[\bar{U}(k)[\ell]: C_{k}^{(3)}\right],
$$

где $c=\nu_{\ell}([k: \mathbf{Q}])$ и $\ell^{r}$ делит степень $[K: \mathbf{Q}]$.

Для любого $\varphi \in \Phi$ мы имеем

$$
\left|\mathrm{Cl}(k)_{\ell, \varphi}\right|=\left\{\begin{array}{lll}
A_{\varphi} & n p u & \varphi \neq \varphi_{0} \\
\ell^{r-c} A_{\varphi} & n p u & \varphi=\varphi_{0}
\end{array}\right.
$$

əде $A_{\varphi}=\left(\mathcal{R}_{k, \varphi}: \mathcal{U}_{k, \varphi}^{(3)}\right)^{-1}\left[\bar{U}(k)[\ell]_{\varphi}: C_{k, \varphi}^{(3)}\right]$.

ДОКАЗАТЕЛЬСТво непосредственно следует из теоремы 9.1 и предложений 9.2 и 9.6 .

Теорема 9.3 приводит к следующему интересному результату.

Tеорема 9.4. Пусть $K=K_{m} u k=\left(K^{+}\right)^{H}$, әде $H$ - циклическая подгруппа группь $G\left(K^{+} / \mathbf{Q}\right)$ такая, что $H \cap T_{r}=\{1\}$ для любого $r \mid \bar{m}, \quad r \neq \bar{m}$, где $T_{r}$ имеет тот же смысл, ито и в теореме 8.1. Пусть $g \geqslant 2$ - число различных простых делителей $m$. Для $\ell \neq 2$ положим $t=\nu_{\ell}(|H|)$. Тогда

$$
\left|\mathrm{Cl}(k)_{\ell}\right| \equiv\left|\mathrm{Cl}(k)_{\ell, \varphi_{0}}\right| \equiv 0 \quad\left(\bmod \ell^{t\left(2^{g-2}-1\right)}\right) .
$$

ДокаЗАТЕЛЬСТво. Мы имеем разложение $\mathcal{U}_{m}=\mathcal{U}_{m}^{+} \oplus \mathcal{U}_{m}^{-}$. Согласно лемме 8.3 группа $\mathcal{U}_{m}^{-}$является когомологически тривиальным $H$-модулем. Тогда из теоремы 8.1 следует, что $\nu_{\ell}\left(\left(\mathcal{U}_{k}^{(3)}: \mathcal{R}_{k}\right)\right)=\nu_{\ell}\left(\left(\mathcal{U}_{k, \varphi_{0}}^{(3)}: \mathcal{R}_{k, \varphi_{0}}\right)\right)=\nu_{\ell}\left(\left(\mathcal{U}_{m}^{+}\right)^{H}: \mathcal{R}_{k}\right)=$ $2^{g-2} t$. Мы знаем, что $\ell^{r-c}$ делит $|H|$. Таким образом, теорема следует из $(9.3)$ и того факта, что индекс $\left[\bar{U}(k)[\ell]_{\varphi}: C_{k, \varphi}^{(3)}\right]$ является цельм числом.

СледСТвИЕ. Пусть $k_{0}$ - максимальное $\ell$-расширение поля $\mathbf{Q}$, содержащееся в $k$, где $k$ имеет тот же смисл, что и в теореме 9.4. Тогда

$$
\left|\mathrm{Cl}\left(k_{0}\right)_{\ell}\right| \equiv 0 \quad\left(\bmod \ell^{t\left(2^{g-2}-1\right)}\right)
$$

Действительно, в этом случае $\left|\mathrm{Cl}\left(k_{0}\right)_{\ell}\right|=\mathrm{Cl}(k)_{\ell, \varphi_{0}}$.

ПреДЛОЖЕНИЕ 9.7. Пусть задано некоторое натуральное $t$. Предположим, что для любого простого $p \mid m$ справедливо сравнение $\varphi\left(p^{e}\right) \equiv 0$ $\left(\bmod \ell^{t}\right)$, где $\ell-$ нечетное простое число и $\varphi\left(p^{e}\right)$ - функция Эйлера, т.е. $p \equiv 1\left(\bmod \ell^{t}\right)$ при $p \neq \ell$ и $е \geqslant t+1$ при $p=\ell$. Тогда существует ииклическая подгруппа $H \subset G\left(K_{m} / \mathbf{Q}\right)$ порядка $\ell^{t}$ такая, что $H \cap T_{r}=\{1\}$ для любого $r \mid \bar{m}, \quad r \neq \bar{m}$.

ДоКАЗАТЕЛЬСТво. Для каждого $p \mid m$ выберем элемент $\sigma_{p} \in T_{p}$, имеющий порядок в точности $\ell^{t}$, и положим $H=\langle\sigma\rangle$, где $\sigma=\prod_{p \mid m} \sigma_{p}$. 
ТЕОРема 9.5. Пусть $K=K_{m}$ - круговое поле такое, что при некотором заданном $t$ выполняется сравнение $\varphi\left(p^{e}\right) \equiv 0\left(\bmod \ell^{t}\right)$ для любого $p \mid m$, где $p^{e}$ - максимальная степень $p$, делящая $m$. Тогда

$$
\left|\mathrm{Cl}\left(K_{m}^{+}\right)_{\ell}\right| \equiv\left|\mathrm{Cl}\left(K_{m}^{+}\right)_{\ell, \varphi_{0}}\right| \equiv 0 \quad\left(\bmod \ell^{t\left(2^{g-2}-2\right)}\right) .
$$

ДокАЗАТЕЛЬСтво. Согласно предложению 9.7 можно найти подполе $k \subset K_{m}^{+}$ такое, что группа $H=G\left(K_{m}^{+} / k\right)$ имеет порядок $\ell^{t}$ и удовлетворяет всем условиям теоремы 9.4. Если порядок группы $\mathrm{Cl}(k)_{\varphi_{0}}$ делится на $\ell^{a}$ для некоторого $a$, то сушествует абелево неразветвленное $\ell$-расширение $M / k$, степень которого делится на $\ell^{a}$, и такое, что $M$ является расширением Галуа поля $\mathbf{Q}$, причем $G(M / k)=$ $G(M / k)_{\varphi_{0}}$. Тогда степень расширения $M K_{m}^{+} / K_{m}^{+}$делит $\ell^{a-t}$. Следовательно,

$$
\left|\mathrm{Cl}\left(K_{m}^{+}\right)\right| \equiv\left|\mathrm{Cl}\left(K_{m}^{+}\right)_{\varphi_{0}}\right| \equiv 0 \quad\left(\bmod \ell^{a-t}\right) .
$$

СЛЕДСТВИЕ. Пусть $\ell$ - нечетное простое число. Если число g различных простых делителей $m$ больше или равно 4 , то поле $K_{m}^{+}$имеет нетривиальную $\ell$-группу классов. Пусть $K_{0}$ - максимальное $\ell$-расширение поля $\mathbf{Q}$, содерәсащеес в $K_{m}^{+}$. Тогда поле $K_{0}$ имеет нетривиальную $\ell$-группу классов.

\section{§10. Заключительные замечания}

Группу $T_{\ell}\left(k_{\infty}\right)_{(0)}$ можно рассматривать как аналог $\ell$-группы классов функционального поля. Таким образом, встает

Вопрос 1. Конечна ли группа $\prod_{\ell} T_{\ell}\left(k_{\infty}\right)_{(0)}$ для вешественного абелева поля $k$, другими словами, верно ли, что группы $T_{\ell}\left(k_{\infty}\right)$ для заданного $k$ тривиальны для всех, кроме конечного числа, $\ell$ ?

Отметим, что доказательство теоремы 3.2 , данное в $\S 3$, кажется довольно сложным. Представляется, что его можно упростить, используя подход Колывагина [6], [12]. С другой стороны, в [3] Гринберг предположил, что модуль $T_{\ell}\left(k_{\infty}\right)$ конечен для любого вполне вещественного поля $k$. Если эта гипотеза справедлива, то теорему 3 можно вывести непосредственно из предложения 4.3. Более того, справедлива

ТЕОРема 10.1. Предположим, что для расиирения $k_{\infty} / k$ справедлива гипотеза Гринберга. Тогда при достаточно большом $n$ для любого промежуточного подполя $k_{n}, k_{\infty} \supset k_{n} \supset k$, мй имеем

$$
T_{\ell}\left(k_{\infty}\right)_{(n)} \cong \widehat{U}_{S}\left(k_{n}\right) / C_{S}\left(k_{n}\right) \text {. }
$$

В связи с гипотезой Гринберга мы докажем одно утверждение, являющееся простым следствием предложения 4.3. Для вещественного абелева поля $k$ мы определим число $i(k)$ как минимальное значение индекса $n$ такое, что группа Галуа $\Gamma_{n}=G\left(k_{\infty} / k_{n}\right)$ действует тривиально на $T_{\ell}\left(k_{\infty}\right)$, т.е. $T_{\ell}\left(k_{\infty}\right)^{\Gamma_{n}}=T_{\ell}\left(k_{\infty}\right)$, где $k_{\infty}-$ круговое $\mathbf{Z}_{\ell}$-расширение поля $k$. Если модуль $T_{\ell}\left(k_{\infty}\right)$ бесконечен, то такого $n$ нет, и в этом случае мы полагаем $i(k)=\infty$.

ПРЕДЛОЖЕНИЕ 10.1. Пусть $k$ - чиклическое вещественное поле алгебраических чисел степени $\ell$ такое, что в $k$ разветвлены $t$ простых чисел $p_{1}, \ldots, p_{t}$, причем $\ell$ остается инертным в $k$. Тогда

$$
i(k) \geqslant \frac{t-1}{\ell}-1 \quad \text { npu } \quad \ell \neq 2, \quad i(k) \geqslant \frac{t}{2}-2 \quad \text { npu } \quad \ell=2 .
$$


ДокАЗАТЕЛЬСтво. Если модуль $T_{\ell}\left(k_{\infty}\right)$ бесконечен, то $i(k)=\infty$. Поэтому достаточно рассмотреть случай, когда $T_{\ell}\left(k_{\infty}\right)$ конечен. Пусть $c$ (соответственно $\left.d\right)$ минимальное число образующих $T_{\ell}\left(k_{\infty}\right)$ (соответственно $\left.\widehat{U}_{S}(k) / C_{S}(k)\right)$ как абелевой $\ell$-группы.

Из теории родов следует, что $c \geqslant t-1$ при $\ell \neq 2$ и с $\geqslant t-2$ при $\ell=2$. Поскольку $d \leqslant \operatorname{rk} \widehat{U}_{S}(k)=\ell$, наше предложение вытекает из следующей леммы.

Лемма 10.1. Пусть $G$ - ииклическая группа порядка $\ell^{n}, A-$-конечная абелева $\ell$-группа, являющаяся $G$-модулем, и а (соответственно $b)$ - минимальное число образующих группь $A_{G}=H_{0}(G, A)$ (соответственно $\left.A^{G}\right)$. Тогда

$$
n \geqslant \frac{a}{b}-1
$$

ДокАЗАтельство. Заметим, что $\left|A_{G}\right|=\left|A^{G}\right|$. Пусть $\alpha: A_{G} \rightarrow A^{G}$ - отображение, индуцированное норменным отображением $N_{G}: A \rightarrow A$. Тогда $|\operatorname{Ker} \alpha| \geqslant$ $\ell^{a-b}$. Поскольку $|\operatorname{Ker} \alpha|=\mid$ Coker $\alpha \mid$ и группа Coker $\alpha=H^{0}(G, A)$ имеет не более $b$ образуюших, мы получаем, что показатель группы $H^{0}(G, A)$ больше или равен $\ell^{\frac{a-b}{b}}$.

Вопрос 2. Справедлива ли теорема 10.1 для произвольного вешественного абелева поля $k$ ?

Вопрос 3. Можно ли обобщить теорему 6.1 на случай относительных абелевых расширений?

Отметим, что такое обобщение, если оно существует, предполагает обобщение понятия гауссовых сумм $g_{\chi}$.

Следует отметить, что теорема 7.1 вместе с приведенным доказательством остается справедливой для любого вполне вешественного поля $k$.

Вопрос 4. Можно ли обобшить теорему 7.1 на случай произвольного поля алгебраических чисел?

Отметим, что мы доказали теоремы 9.4 и 9.5 , используя вычисления некоторых групп когомологий модуля $U$. Можно спросить, существуют ли другие интересные примеры подгрупп $H \subset G\left(K_{m} / \mathbf{Q}\right)$, приводящие к каким-либо другим нетривиальным условиям делимости для групп классов круговых полей?

Наконец, отметим, что наиболее интересна задача обобщения результатов настоящей работы на другие типы полей, обладаюших системами специальных единищ. Например, для абелевых расширений мнимых квадратичных полей.

\section{Список литературы}

1. Cornell $G$. Exponential growth of the $\ell$-rank of the class group of the maximal real subfield of cyclotomic fields // Bull. Amer. Math. Soc. 1983. V. 8. № 1. P. 55-58.

2. Gras G. Classes d'ideaux des corps abélienn et nombres de Bernoulli généralisés // Ann. Inst. Fourier. 1977. V. 27. P. 1-66.

3. Greenberg $R$. On the Iwasawa invariants of totally real number fields // Amer. J. Math. 1976. V. 98. № 1. P. 263-284.

4. Greither C. Class groups of abelian fields, and the main conjecture // Ann. Inst. Fourier. 1992. V. 42. P. 449-499.

5. Iwasawa $K$. Lectures on $p$-adic $L$-functions. Princeton: Princeton Univ. Press and Univ. of Tokyo Press, 1972.

6. Kolyvagin V.A. Euler systems // The Grothendieck Festschrift. V. 2. P. 435-483. Birkhäuser Verlag, 1990. 
7. Kubert D.S. The universal ordinary distribution // Bull. Soc. math. France. 1979. V. 107. P. 179-202.

8. Kubert D.S. The $\mathbf{Z} / 2 \mathbf{Z}$ cohomology of the universal ordinary distribution // Bull. Soc. math. France. 1979. V. 107. P. 203-224.

9. Кузьмин Л.В. Модуль Тэйта полей алгебраических чисел // Изв. АН СССР. Сер. матем. 1972. Т. 36. № 2. С. 267-327.

10. Кузьмин Л.В. Некоторые теоремы двойственности для круговых Г-расширений полей алгебраических чисел СМ-типа // Изв. АН СССР. Сер. матем. 1979. Т. 43. №3. C. $483-546$.

11. Кузьмин Л.В. Некоторые явные вычисления в локальных и глобальных круговых полях // Тр. Матем. ин-та РАН. 1995. Т. 208. С. 202-223.

12. Lang S. Cyclotomic fields I, II, combined 2-nd edition Grad. Texts in Math. V. 121. New York: Springer-Verlag, 1990.

13. Mazur B., Wiles A. Class fields of abelian extensions of Q // Invent. math. 1984. V. 76. P. 179-330.

14. Rubin K. Global units and ideal class groups // Invent. Math. 1987. V. 89. P. 511-526.

15. Sinnott $W$. On the Stickelberger ideal and the circular units of a cyclotomic field // Ann. of Math. 1978. V. 108. P. 107-134.

16. Sinnott $W$. On the Stickelberger ideal and the circular units of an abelian field // Invent. math. 1980. V. 62. P. 181-234.

17. Wiles A. The Iwasawa conjecture for totally real fields // Ann. of Math. 1990. V. 131. P. 493-540.

Российский научный центр "Курчатовский институт"

Институт Информационных технологий

Поступило в редакцию

Москва 123182, Россия

14.XI.1995

E-mail: kuzmin@ofpnp.kiae.su 